\title{
WestVirginiaUniversity
}

THE RESEARCH REPOSITORY @ WVU

Graduate Theses, Dissertations, and Problem Reports

2000

\section{Characterization and applications of pulsed millisecond glow discharge sources}

Cris Lee Lewis

West Virginia University

Follow this and additional works at: https://researchrepository.wvu.edu/etd

\section{Recommended Citation}

Lewis, Cris Lee, "Characterization and applications of pulsed millisecond glow discharge sources" (2000). Graduate Theses, Dissertations, and Problem Reports. 1234.

https://researchrepository.wvu.edu/etd/1234

This Dissertation is protected by copyright and/or related rights. It has been brought to you by the The Research Repository @ WVU with permission from the rights-holder(s). You are free to use this Dissertation in any way that is permitted by the copyright and related rights legislation that applies to your use. For other uses you must obtain permission from the rights-holder(s) directly, unless additional rights are indicated by a Creative Commons license in the record and/ or on the work itself. This Dissertation has been accepted for inclusion in WVU Graduate Theses, Dissertations, and Problem Reports collection by an authorized administrator of The Research Repository @ WVU.

For more information, please contact researchrepository@mail.wvu.edu. 


\title{
Characterization and Applications of Pulsed Millisecond Glow Discharge Sources
}

\author{
Cris L. Lewis \\ Dissertation Submitted to the \\ Eberly College of Arts and Sciences \\ at West Virginia University \\ in partial fulfillment of the requirements \\ for the degree of \\ Doctor of Philosophy \\ in \\ Chemistry \\ Fred L. King, Ph.D., Chair \\ Ronald B. Smart, Ph.D. \\ Aaron Timperman Ph.D. \\ Earl E. Scime, Ph. D. \\ Paul W. Jagodzinski, Ph.D. \\ Morgantown, West Virginia \\ 2000
}

Keywords: Glow Discharge, Plasma, lonization, Copyright 2000 Cris L. Lewis 


\section{Abstract \\ Characterization and Applications of Pulsed Millisecond Glow Discharge Sources Cris L. Lewis}

Most modern analytical techniques are derived from coupled methods involving a chromatographic separation followed by mass spectrometric detection. These techniques are employed in modern laboratories in the form of gas and liquid chromatography mass spectrometric systems. Chromatography methods separate and concentrate different chemical species based on phase partitioning. Once separated, the sample is analyzed mass spectrometrically, where further information and identification of the analyte may be deduced based on its inherent mass-to-charge ratio. However, following chemical separation and previous to any mass spectrometric detection, the analyte most be ionized. To date there are numerous possible methods for ionization, however, they can be classified into hard and soft ion sources.

The common ionization method is electron ionization $(\mathrm{EI})$ in which the compound is atomized or fragmented. This method readily provides both elemental and structural information, because compounds of the same class will have similar elemental compositions and fragmentation patterns. In contrast, soft ionization, such as chemical ionization $(\mathrm{Cl})$, yields molecular weight information because the unfragmented molecule is ionized and detected intact. It is easy to see that these two methods provide complementary information. To date, no ion source has been developed that grants both $\mathrm{El}$ and $\mathrm{Cl}$ characteristics simultaneously and independently of one another.

In these investigations the pulsed glow discharge source has demonstrated an ability to permit the separation of electron-ionized species from 
Penning ionized species. Penning ionization is a relatively soft ionization process, where analyte molecules are ionized by collisions with metastable argon atoms. In pulsed glow discharge plasmas these two processes are separated both temporally and spatially. The analytical advantage of the separation of these two processes can be illustrated in the discrimination of isobaric interferences using time-gated detection, an example would be the determination of ${ }^{40} \mathrm{Ca}^{+}$from ${ }^{40} \mathrm{Ar}^{+}$. Further exploitation of these temporal regimes can be applied to molecular analysis, where concurrent molecular and structural information can be obtained. 


\section{Acknowledgments}

I would like to take this opportunity to thank my advisor Professor F. L. King his guidance over the course of this decade. Through his patience and tutelage I have learned independence and the systematic approach of being a scientist. Through his confidence in me I have grown more independent and self reliant in my ideas and abilities. I would also like to thank the Dr. Vahid Majidi and the staff of the Chemistry Science and Technology Division 9 (CST-9) at Los Alamos National Laboratory, where a significant part of this work materialized.

Also, I would like to thank my committee members, for their advice and suggestions on the preparation of this thesis. The staff and faculty for their help and knowledge passed on to me over these few years. I would also like to make a special acknowledgement to Donald Feathers and Allen Burns for their patience, professional skills and engineering feats. And how can I forget my graduate peers for making this possibly the most enjoyed time of my life.

Foremost, I would like to express my appreciation to my parents Oswald and Barbara Lewis for their encouragement and continued support throughout this and all of my life's endeavors. Without their love and encouragement I would not be where I am today. 


\section{Table of Contents}

CHARACTERIZATION AND APPLICABILITY OF PULSED MILLISECOND

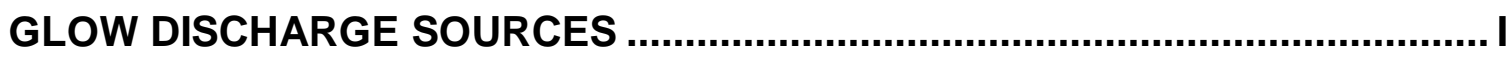

ABSTRACT

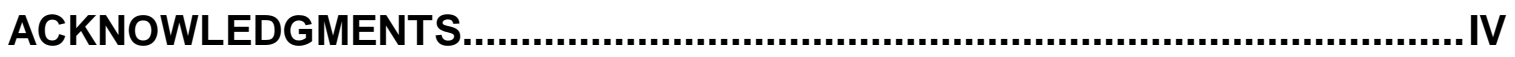

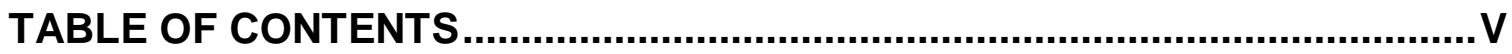

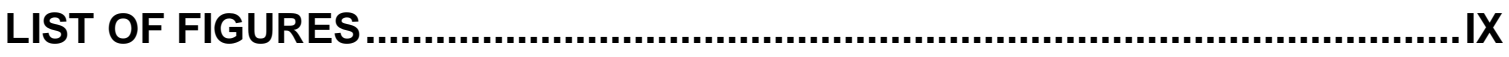

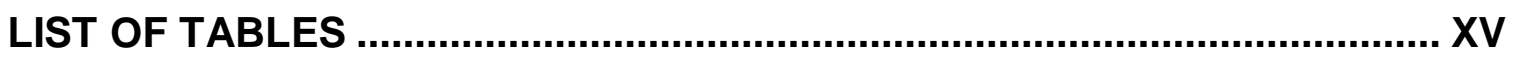

1 INTRODUCTION ........................................................................................ 1

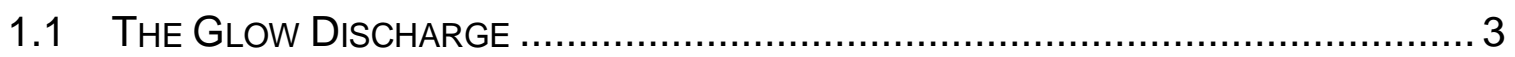

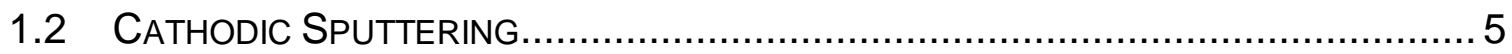

1.3 EXCITATION AND IONIZATION PROCESSES ............................................. 7

1.4 Pulsed Operation of THE Glow Discharge ........................................... 11

2 INSTRUMENTATION ................................................................................. 15

2.1 PuLSED GLOW Discharge ION SOURCE ................................................... 15

3 SPECTRAL, SPATIAL, AND TEMPORAL CHARACTERIZATION OF A

MILLISECOND PULSED GLOW DISCHARGE: OPTICAL

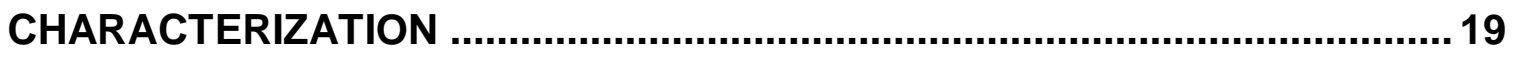

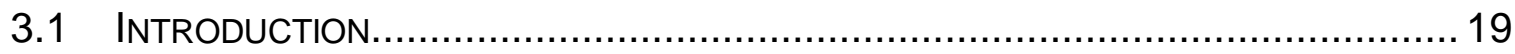




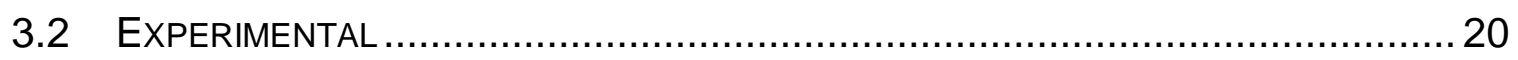

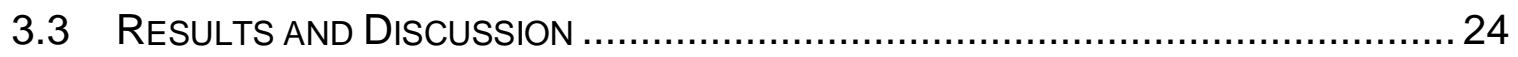

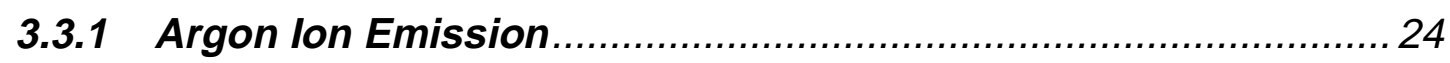

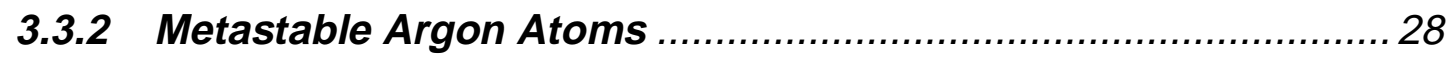

3.3.3 Sputtered Analyte Emission: Copper Atom Transitions ............. 35

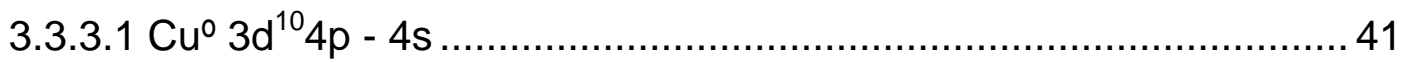

3.3.3.2 Cu High Energy States.......................................................... 41

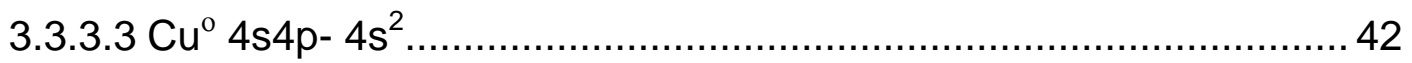

3.3.3.4 Afterpeak-to-plateau emission intensity ratio .............................. 43

3.3.3.5 Spatial Maps of Atomic Emission and Absorbance of Sputtered

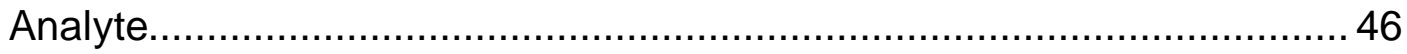

3.3.3.5.1 Ground State Copper Atom Absorbance Maps.................... 46

3.3.3.5.2 Copper Atom Emission Maps............................................ 49

3.3.4 Classification of Copper Ion Electronic States.......................... 54

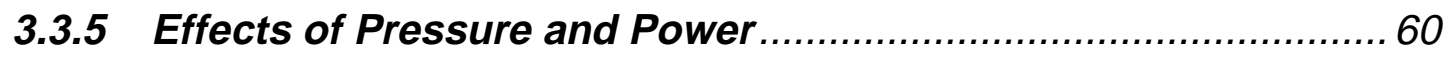

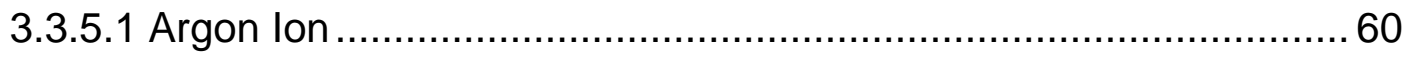

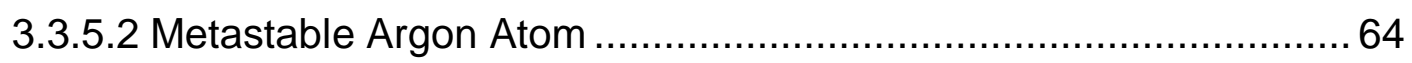

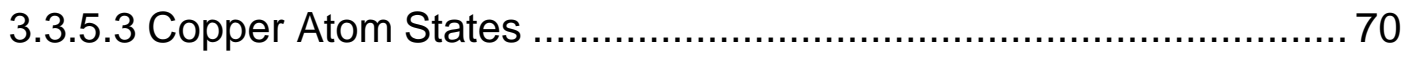

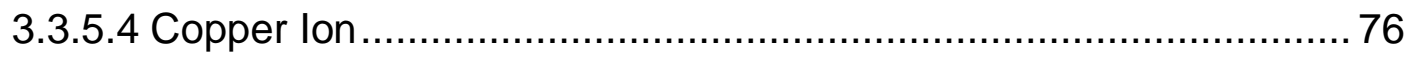

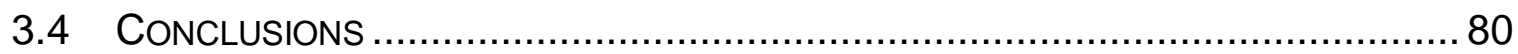

4 SPECTRAL, SPATIAL, AND TEMPORAL CHARACTERIZATION OF A MILLISECOND PULSED GLOW DISCHARGE: DIFFUSION EFFECTS ........... 82

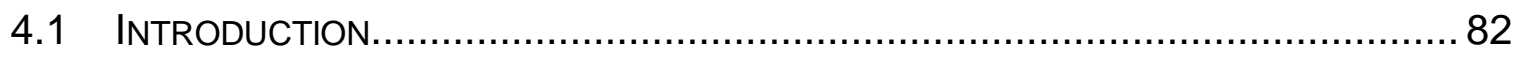




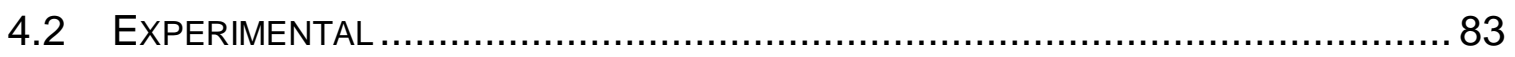

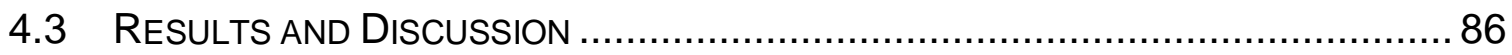

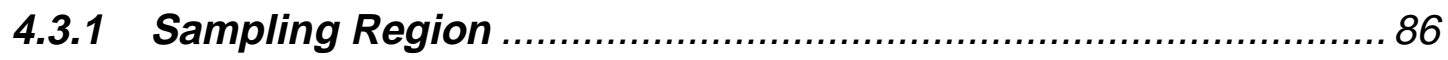

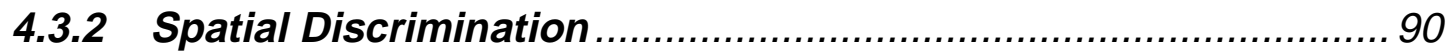

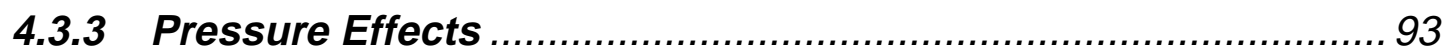

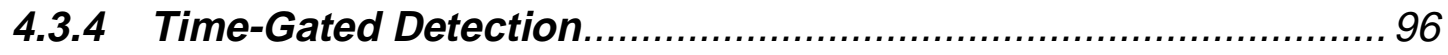

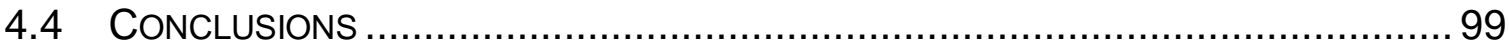

5 TEMPORAL SEPARATION OF ${ }^{40} \mathrm{CA}^{+}$FROM ${ }^{40} \mathrm{AR}^{+}$IN TIME GATED GLOW DISCHARGE USING MASS SPECTROMETRY ...........................................100

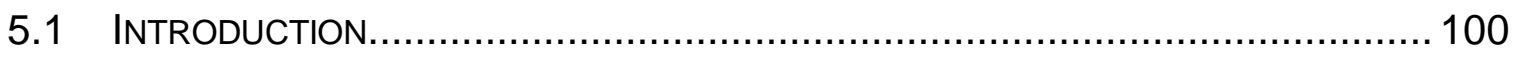

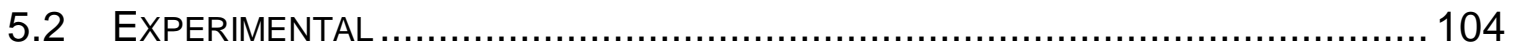

5.2.1 Sample Preparation ................................................................ 107

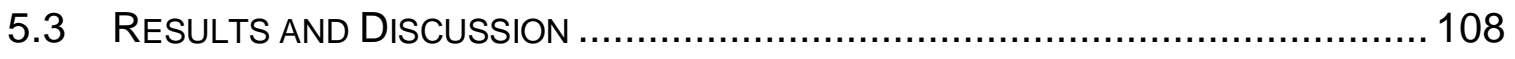

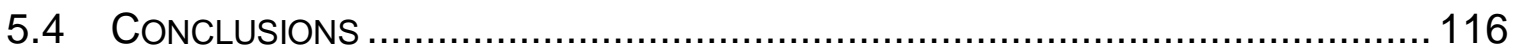

6 DETECTION OF VOLATILE ORGANIC COMPOUNDS USING THE PULSED GLOW DISCHARGE ION SOURCE COUPLED WITH A TIME-OF-

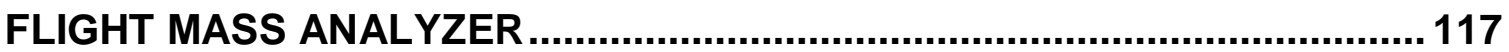

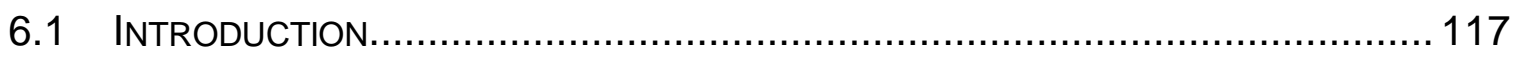

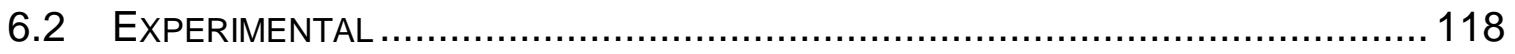

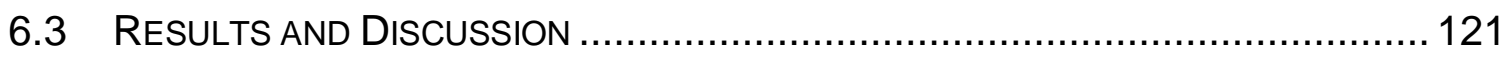

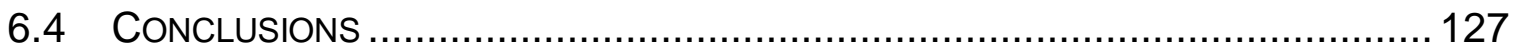




\section{CHARACTERIZATION OF A PULSED GLOW DISCHARGE LASER}

ABLATION SYSTEM USING OPTICAL EMISSION ......................................128

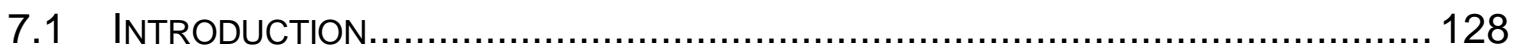

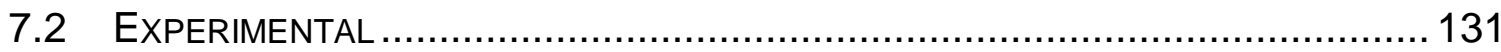

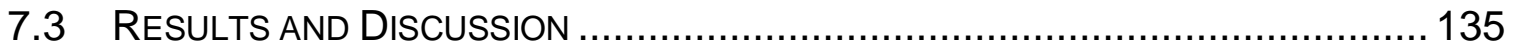

7.3.1 Part I. Transport of Ablated Material ............................................ 140

7.3.2 Part II. Glow Discharge Metastable Distributions............................ 144

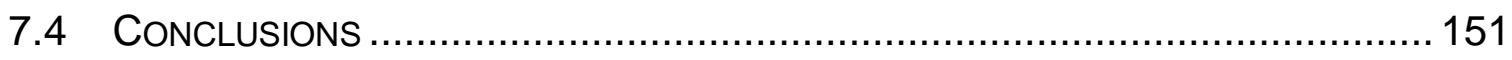

VITA 


\section{List of Figures}

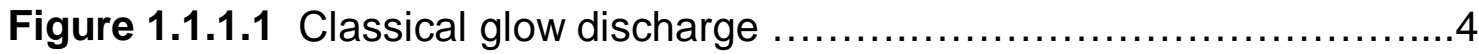

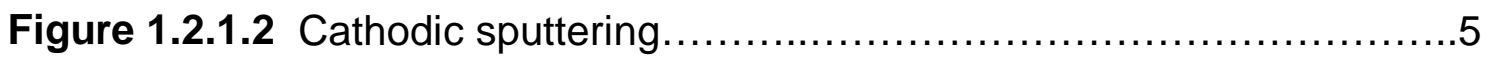

Figure 1.3.1.3 Collisional ionization processes..............................10

Figure 1.4.1.4 Temporal ion signals of pulsed plasmas.......................13

Figure 2.1.1.1 The glow discharge ion source...............................16

Figure 2.1.1.2 Diagram of a direct insertion probe...........................17

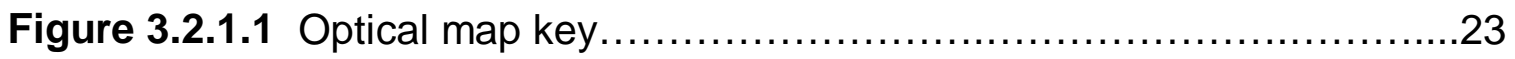

Figure 3.3.1.1 Two-dimensional plot of argon ion emission monitored at different wavelengths as a function of distance ....................................26

Figure 3.2.1.2 Temporal emission profiles of argon ion monitored at $476.4 \mathrm{~nm}$, at different wavelengths as a function of distance ..........................26

Figure 3.2.1.3 Two-dimensional maps argon ion emission monitored at 476.4

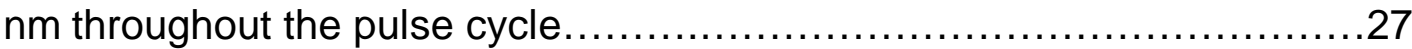

Figure 3.3.2.1 (A) Two-dimensional plot of metastable argon atom emission intensity, $811.5 \mathrm{~nm}$, as a function of distance, with (B) the collected emission

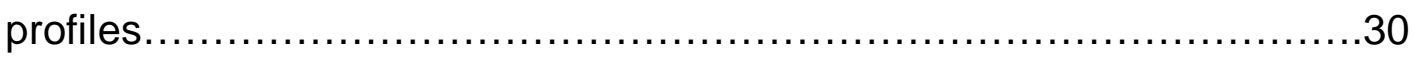

Figure 3.3.2.2 (A) Two-dimensional plot of metastable argon atom absorption intensity, $811.5 \mathrm{~nm}$, as a function of distance, with (B) the collected absorption profiles as a function of distance.

Figure 3.3.2.3 Two-dimensional maps metastable argon emission at $811.5 \mathrm{~nm}$ throughout the pulse cycle. 
Figure 3.3.2.4 Two-dimensional maps metastable argon absorption at $811.5 \mathrm{~nm}$ throughout the pulse cycle................................................

Figure 3.3.3.1 Energy level diagram highlighting copper atoms transitions of interest labeled $A$ through $F$ .38

Figure 3.3.3.2 Temporal emission profiles of copper atom transitions depicted in

Figure 3.3.3.2, labeled A - C 39

Figure 3.3.3.3 Temporal emission profiles of copper atom transitions depicted in

Figure 3.3.3.2, labeled D - F 40

Figure 3.3.3.4 Calculated afterpeak-to-plateau ratio as a function of increasing distance for various copper atom transitions. 45

Figure 3.3.3.5 Two-dimensional absorption maps of the copper atom population monitored throughout the pulse cycle 48

Figure 3.3.3.6 Two-dimensional emission maps of a low-lying copper atom transition, $3.82 \mathrm{eV}$, monitored throughout the pulse cycle .52

Figure 3.3.3.7 Two-dimensional emission maps of a high-lying copper atom transition, $7.18 \mathrm{eV}$, monitored throughout the pulse cycle .53

Figure 3.3.4.1 Energy level diagram highlighting copper ion transitions of interest labeled $\mathrm{A}$ and $\mathrm{B}$. .55

Figure 3.3.4.2 Temporal profiles and emission maps of copper ion transitions of interest labeled from Figure 3.3.4.1 .56

Figure 3.3.4.3 Temporal profiles and emission maps of copper ion emission monitored at $224.7 \mathrm{~nm}$ 
Figure 3.3.5.1 Two-dimensional emission maps of argon ion emission, 476.4 $\mathrm{nm}$, at a constant power of 1.5 watts and pressures of $(\mathrm{A}) 0.5$, (B) 0.8 , and

(C) 1.0 Torr 62

Figure 3.3.5.2 Two-dimensional emission maps of argon ion emission, 476.4 $\mathrm{nm}$, at a constant pressure of 0.8 torr and powers of $(\mathrm{A}) 0.5,(\mathrm{~B}) 1.3$, and $(\mathrm{C})$ 2.3 watts. 63

Figure 3.3.5.3 Two-dimensional emission maps of metastable argon atom, $811.5 \mathrm{~nm}$, at a constant power of 1.5 watts and pressures of $(A) 0.5,(B) 0.8$, and (C) 1.0 Torr 66

Figure 3.3.5.4 Two-dimensional raw emission maps of metastable argon atom, $476.4 \mathrm{~nm}$, at a constant power of 1.5 watts and pressures of $(A) 0.5,(B) 0.8$, and (C) 1.0 torr 67

Figure 3.3.5.5 Two-dimensional emission maps of metastable argon atom, $811.5 \mathrm{~nm}$, at a constant pressure of 0.8 torr and powers of $(\mathrm{A}) 0.5,(\mathrm{~B}) 1.3$, and (C) 2.3 watts. 68

Figure 3.3.5.6 Two-dimensional raw emission maps of metastable argon atom, $811.5 \mathrm{~nm}$, at a constant pressure of 0.8 torr and powers of $(A) 0.5,(B) 1.3$, and (C) 2.3 watts 69

Figure 3.3.5.7 Two-dimensional emission maps of a low-lying copper atom transition, $3.82 \mathrm{eV}$, at a constant power of 1.5 watts and pressures of $(\mathrm{A}) 0.5$, (B) 0.8 , and (C) 1.0 torr 72 
Figure 3.3.5.8 Two-dimensional emission maps of a high-lying copper atom transition, $7.18 \mathrm{eV}$, at a constant power of 1.5 watts and pressures of $(\mathrm{A}) 0.5$, (B) 0.8 , and (C) 1.0 torr 73

Figure 3.3.5.9 Two-dimensional emission maps of a low-lying copper atom transition, $3.82 \mathrm{eV}$, at a constant pressure of 0.8 torr and powers of $(\mathrm{A}) 0.5$,

(B) 1.3 , and (C) 2.3 watts. 74

Figure 3.3.5.10 Two-dimensional emission maps of a high-lying copper atom transition, $7.12 \mathrm{eV}$, at a constant pressure of 0.8 torr and powers of $(\mathrm{A}) 0.5$, (B) 1.3 , and (C) 2.3 watts. .75

Figure 3.3.5.11 Two-dimensional emission maps of copper ion emission, 224.7 $\mathrm{nm}$, at a constant power of 1.5 watts and pressures of $(\mathrm{A}) 0.5,(\mathrm{~B}) 0.8$, and (C) 1.0 torr 78

Figure 3.3.5.12 Two-dimensional emission maps of copper ion emission, 224.7 $\mathrm{nm}$, at a constant pressure of 0.8 torr and powers of $(\mathrm{A}) 0.5,(\mathrm{~B}) 1.3$, and $(\mathrm{C})$ 2.3 watts. 79

Figure 4.3.1.1 Temporal ion profiles of argon ion at increasing distances......87

Figure 4.3.1.2 Temporal ion profiles of sputtered analyte, ${ }^{56} \mathrm{Fe}^{+}$, at increasing

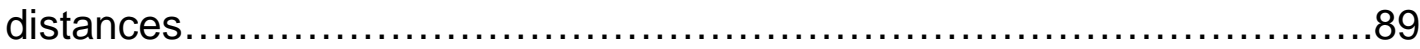

Figure 4.3.2.1 Overlapping ion profiles of sputtered analyte and background gas with increasing distances. 92

Figure 4.3.3.1 Overlapping ion profiles of sputtered analyte, ${ }^{56} \mathrm{Fe}^{+}$, at increasing distances for (A) 0.5 and (B) 0.8 torr .94 
Figure 4.3.3.2 Overlapping ion profiles of background gas, ${ }^{40} \mathrm{Ar}^{+}$, at increasing distances for (A) 0.5 and $(\mathrm{B}) 0.8$ torr...... .95

Figure 4.3.4.1 Time-gated detection of an iron sample taken during the plateau, $4.8 \mathrm{~ms}$, and afterpeak $8.0 \mathrm{~ms}$. 97

Figure 4.3.4.2 Temporal ion profiles of ${ }^{56} \mathrm{Fe}^{+}$and applied power pulses at very large distances .98

Figure 5.3.1.1 A) Temporal ion profiles and B) mass spectrum acquired at

$4.8 \mathrm{~ms}$ 110

Figure 5.3.1.2 A) Temporal ion profiles and B) mass spectrum acquired at $6.6 \mathrm{~ms}$

Figure 5.3.1.3 Average spectrum of five coadded scans obtained for $0,50,100$, 150, and 200 ppm Ca doped iron standards using the modulated (A) dc glow discharge and gate delay at $7.0 \mathrm{~ms}$, and (B) rf glow discharge source using a delay gate positioned at $6.8 \mathrm{~ms}$ 113

Figure 5.3.1.4 Calibration curves for calcium standards using a $(\mathbf{A})$ dc glow discharge, and (B) rf glow discharge source. 115

Figure 6.3.1.1 Illustration of the glow discharge pulse sequence and temporal regions of interest 121

Figure 6.3.1.2 Prepeak spectra, taken at $0.3 \mathrm{~ms}$, (a) with and (b) without the addition of $\mathrm{p}$-xylene. 123

Figure 6.3.1.3 Comparison (a) electron ionization spectra of a NIST p-xylene with the (b) prepeak spectrum of $p$-xylene using the glow discharge.......124 
Figure 6.3.1.4 Afterpeak spectra, taken at $6.5 \mathrm{~ms}$, (a) with and (b) without the

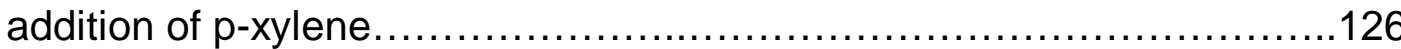

Figure 7.2.1.1 Experimental setup for the laser ablation glow discharge measurements. 134

Figure 7.3.1.1 a) Temporal profile of the $2 \mathrm{~ms}$ glow discharge power pulse, with respect to the monitored copper atom emission without (b) power application to the glow discharge source and (c) with the application of voltage.......136

Figure 7.3.1.2 a) Temporal emission profiles of copper atom emission, 368.7 $\mathrm{nm}$, monitored at series of laser delays (b) in the absence and (c) presence of the applied to the glow discharge source. 138

Figure 7.3.1.3 Afterpeak intensity as a function of laser target distance at various laser delays and pressures. 143

Figure 7.3.2.1 Emission temporal profiles of copper atom emission for a series of pressure at given cathode distance. 146

Figure 7.3.2.2 Afterpeak intensity as a function of cathode distance at various pressures. 148

Figure 7.3.2.3 Afterpeak temporal position as a function of cathode distance at various pressures 148

Figure 7.3.2.4 Expanded views of copper atom emission profiles at increasing cathode distance 150 


\section{List of Tables}

Table 3-1 Argon ion emission intensities taken during the

plateau and afterpeak...........................................25

Table 3-2 Copper atom emission intensities taken during the

plateau and afterpeak............................................

Table 6-1 Operating conditions of the time of flight mass analyzer ..........121 


\section{Introduction}

Since the dawn of time, mankind has pondered upon the nature of the universe in which he lives. Early Greek philosophers began this quest of knowledge through a series of hypotheses involving the composition of matter. It was concluded that all matter was comprised of one basic element and differed only by the relative amount of this element present. Later Empedocles theorized not one but four elements, which took the notorious shapes of earth, wind, fire and water. However a philosopher named Democritius found these explanations lacking substance, and envisioned not one or four, but several different elements. He reasoned that matter consisted of tiny invisible particles that he called "atoms" 1,2 .

Since experimentation has caught up with Democritius's vision, mankind has identified and characterized over 105 elements. Generally, characterization and identification of the elements has primarily been accomplished by optical spectroscopy. These techniques measure and interpret electromagnetic radiation that is absorbed or emitted by atomic species that are characteristic of an element's identity. Such techniques include atomic absorption spectrometry, atomic emission spectrometry, and atomic fluorescence spectrometry.

An alternative approach to these optical techniques lies in mass spectrometry, in which analytes are atomized, ionized, and then identified based on their characteristic mass-to-charge ratio $(\mathrm{m} / \mathrm{z})$. Generally ionization is accomplished through pseudo-controlled processes that ionize gas phase atoms by the removal or addition of an electron. The mass-to-charge ratio is the ratio of 
the mass of the atom or molecule to the number of deficient or surplus electrons that have been lost or gained during ionization. The ions are separated according to their mass-to-charge ratio using electrostatic or magnetic fields. The principal advantage of this type of analysis is that there are far fewer isotopes than electronic transitions. Based on the type of sample and the information needed, a mass spectrometrist determines the appropriate ionization source and mass analyzer combination.

In the realm of elemental analysis, glow discharge sources have become recognized as stable atom and emission sources for the spectrometeric determination of solid elemental composition ${ }^{3}$. A glow discharge is a plasma formed by the electrical breakdown of an inert gas at low pressures. Species within the plasma are excited or ionized through a series of collisional events. Traditionally, glow discharges were used as line sources for atomic spectroscopy measurements. Ironically, however, the roots of glow discharge sources coincide with the development of the first mass spectrometer by J.J. Thompson. ${ }^{4}$ In the 1970s Coburn and Kay revitalized Thompson's earlier experiment when they successfully monitored the ions produced by a glow discharge source using a modern quadrupole mass analyzer to obtain information regarding a sample's elemental composition. ${ }^{5}$ Later investigators in the 1980 s would refine this technique for the direct trace elemental analyses of solid samples. 


\subsection{The Glow Discharge}

A classical description of a glow discharge involves two electrodes immersed in a low-pressure inert gas medium of $0.1-10$ torr. The application of a dc potential across the two electrodes results in the breakdown of the inert gas, and the development of an electrical discharge or plasma. This plasma is a result of electrons within the medium accelerating toward the positively charged anode. During this migration, collisions with gas species may occur and result in ionization/excitation through a process known as electron ionization. Once formed, positively charged ions begin to accelerate toward the negatively biased cathode. Subsequent collisions during this migration may propagate more electrons and ions. The ultimate fates of these ions are collisions with the cathode surface, producing photons, ions, secondary electrons, and the release of neutral atoms. Used primarily as an ion source for solids analysis, glow discharges have also been used for solution residue analysis and as a detection device for gas chromatography. ${ }^{6}$ In most analytical configurations the sample to be analyzed serves as the cathode while the anode consists of the discharge chamber.

The two characteristic spatial regions existing within the glow discharge, the cathode dark space and the negative glow, are shown in Figure 1.1.1.1 The cathode dark space is termed such because of its low level of luminosity. In regions near the cathode surface nearly all the potential drop between the two electrodes occurs. This potential drop corresponds to the electric field "repulsive push" (attractive pull) of electrons (positively charged ions) from the negatively 
charged cathode surface. This field extends out from and rapidly diminishes at increasing distance from the cathode. Because of the push, the kinetic energy of electrons in this region is too high for significant cross sections for collisional excitation to exist; hence, no excitation and no luminescence. By contrast, the negative glow region is defined by its high luminosity, this region is essentially field free allowing charged particles to diffuse freely. Energetic electrons that have been slowed though elastic collisions possess a significant cross section for the collisional excitation of atoms or recombination with ions to generate photons. These two regions spatially decouple atomization and excitation/ionization processes as well as define their occurrence.
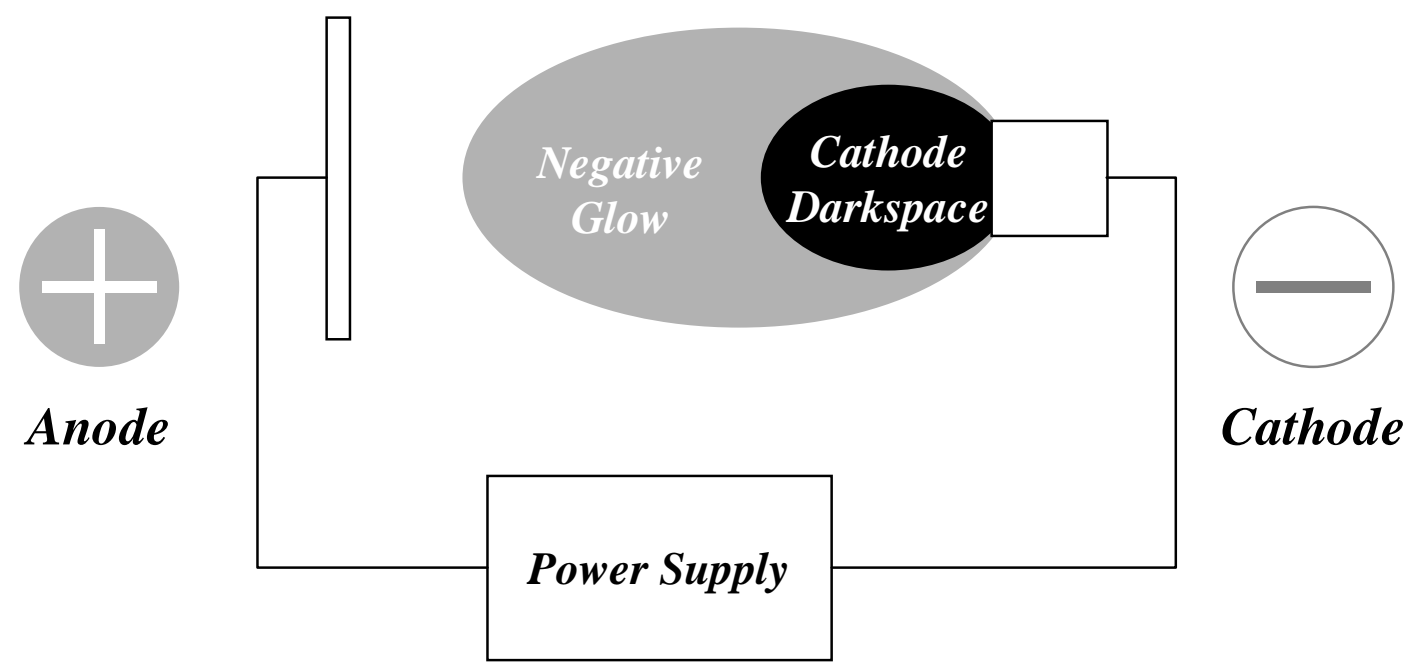

Figure 1.1.1.1 Illustration of a simple glow discharge device. 


\subsection{Cathodic Sputtering}

Analyte atom production occurs through the bombardment of the cathode surface with highly energetic positive ions. Positive ions formed by the discharge process acquire kinetic energy as they migrate across the cathode darkspace. Upon impact with the cathode surface, an ion's kinetic energy is transferred into the cathode lattice. If this energy is great enough to exceed lattice binding energy, particles are ejected, or "sputtered", from the surface as illustrated in Figure 1.2.1.1. The population of sputtered species consists largely of neutral atoms with a small percentage of secondary ions and secondary electrons. Sputtered ions quickly redeposit, while secondary electrons propagate the discharge processes. Neutral atoms, however, diffuse across the cathodic dark space and into the negative glow where they can undergo a variety of excitation or ionization processes.

Great analytical importance lies in the fact that the sputtered atom population must be representative of the sample's atomic composition. This relation relies on the establishment of a sputtering equilibrium, termed preburn period, prior to any measurements. Within this time, any differential

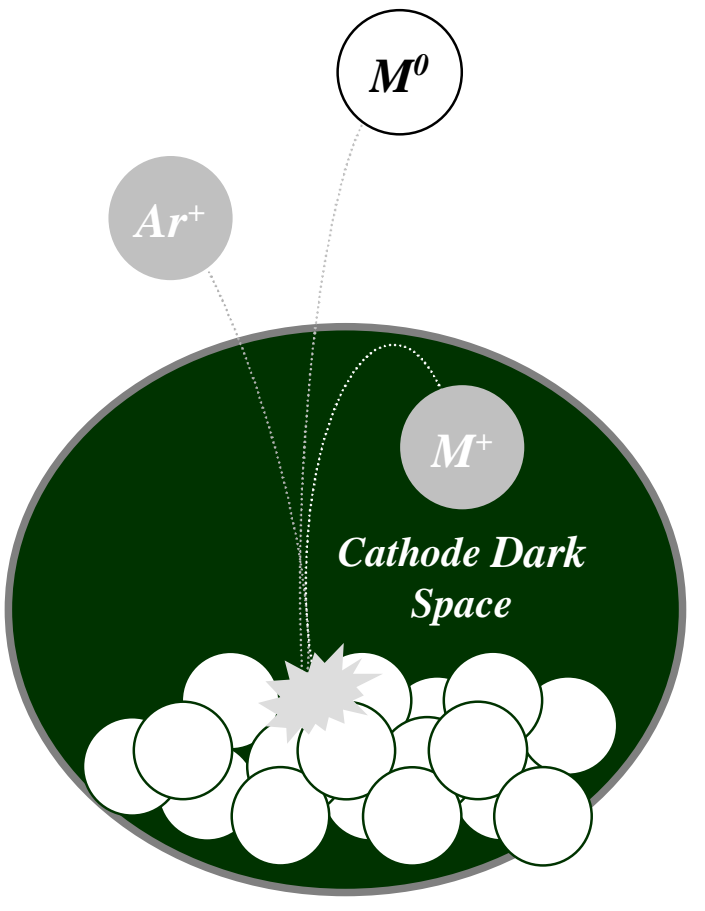

Figure 1.2.1.1.Cathodic sputtering 
sputtering of major cathodic components is offset by an enrichment of lower sputter yield components on the surface. Within a short period of time, an equilibrium will develop between preferentially sputtered material and the enriched sample surface. Continued sputtering will release the enriched surface atoms. 


\subsection{Excitation and lonization Processes}

It has been well documented that atomization and excitation/ionization processes occur in distinct spatial regions of the glow discharge ${ }^{7}$. Atomization occurs inside the cathode dark space at the cathode surface, whereas excitation and ionization occur in the negative glow region. Species within the negative glow transfer energy through collisions that may result in excitation, or in some cases ionization of one or more of the collision partners.

Upon power initiation electrons and ions accelerate and quickly acquire kinetic energy as a result of the electric field established between the cathode and the surrounding anode. The magnitude of the kinetic energy acquired is a function of discharge parameters, such as the pressure of the discharge gas and the magnitude of applied potential. The pressure defines the number of collisions a species will undergo during this migration. The applied potential defines the kinetic energy that can be obtained by the charged particles. When species collide there are two types of collision processes that can occur, elastic and inelastic. Elastic collisions redistribute kinetic energy between the collision partners. This process is responsible for the thermalization or dampening of a species net kinetic energy. In contrast, an inelastic collision partitions a portion of the resultant collision energy into one or more of the collision partners as potential or internal energy. If this energy is great enough, excitation or ionization of one of the collision partners is possible. Considerable importance is placed on collisions resulting in ionization during mass spectrometric analyses ${ }^{8}$. Primarily there are two types of inelastic collisions responsible for the generation of more 
than $90 \%$ of all excited and ionized species within glow discharges: electron ionization and Penning ionization ${ }^{9}$.

Inelastic collisions involving highly energetic electrons colliding with a gas phase species can result in electron excitation and ionization of the atom or molecule involved in the collision, Figure 1.3.1.1 (i). As the electron's kinetic energy exceeds than the lowest ionization potential of its collision partner, ionization becomes important. The average energy of the electron population found within glow discharges is $2-4 \mathrm{eV}$. Of this population only a small fraction (.15\%) are actually capable of ionizing the common discharge gas, of argon, having an ionization potential of $15.76 \mathrm{eV}$.

Recombination of discharge ions with thermal electrons yields long-lived excited species, known as metastables atoms. Metastable atoms are incapable of relaxing to a ground state by emission of a photon because the required transition is quantum mechanically forbidden. Relaxation is dependent upon inelastic collisions that are required to release this internal energy. Collisions with a species allows the potential energy within the metastable atom to be released. This can result in ionization of the collision partner when its ionization potential is less than the energy of the metastable state. This process is known as Penning ionization, Figure 1.3.1.1 ii. Penning ionization may account for 40$80 \%$ of all sputtered atom ionization in analytical glow discharges plasmas. ${ }^{10}$ In argon, the metastable states of ${ }^{3} \mathrm{P}_{2}$ and ${ }^{3} \mathrm{P}_{0}$ occur with energies at $11.52 \mathrm{eV}$ and $11.72 \mathrm{eV}$. This energy is great enough to ionize a majority of the elements within the Periodic Table without ionizing may of the atmospheric impurities present in 
the discharge. This affords some discrimination is afforded between analyte and background impurity specific in GDMS.

Other collisional processes that can be responsible for sputtered atom ionization in the negative glow region are depicted in Figure 1.3.1.1. Collisions involving ionized species may transfer an electron to the target species, resulting in a kinetically energetic neutral and a thermalized ion. These processes can be between identical element species having identical ionization potentials and is known as symmetric charge exchange (iii); or can be between different ion/neutral pairs where the ionization potential of the target is less than that of its collisional partner, known as nonsymmetric (iv) charge exchange. Both symmetric and nonsymmetric charge exchange account for only a small percentage of the total ion population in analytical GD plasmas. Other collisions involving ionized species may produce polyatomic ions. Associative (v) and three-body (vi) collisions may account for only a fraction of the total ion population, but are the origin of many isobaric interferences found in glow discharge mass spectrometry. 


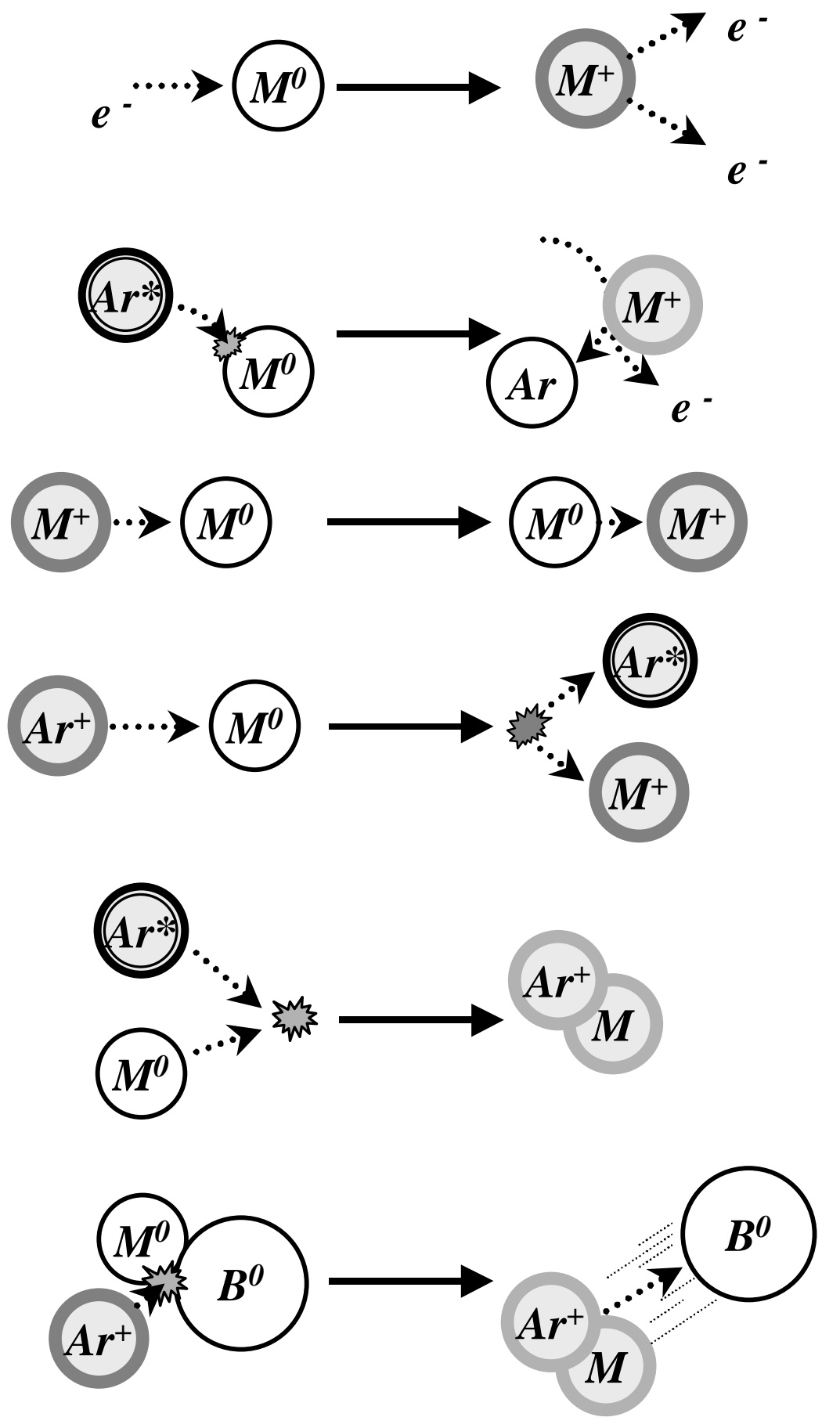

(i)

Figure 1.3.1.1 Collisional ionization processes occurring within the negative glow include (i) electron ionization, (ii) Penning ionization, (iii) resonant charge exchange, (iv) nonresonant charge exchange, (v) associate ionization, and (vi) and three body collisions. Adapted from reference $^{9}$ 


\subsection{Pulsed Operation of the Glow Discharge}

Steady state discharges rely on a constant potential being applied to the cathode at all times. As ions and high-energy neutrals bombard the sample, the cathode begins to heat. Such heating leads to a higher resistance and an annealing effect. These results can lead to sample cracking, discharge arcing, or the preferential sputtering of the sample matrix. ${ }^{11}$ Methods to alleviate resistive heating in steady state discharges are limited to working under lower potentials, changing cathode geometry, or water-cooling the cathode. An alternative to applying a steady or continuous potential is to modulate the potential. By applying potentials in modulated cycles consisting of short pulses, the cathode is allowed to cool and higher instantaneous voltages and currents can be applied. Higher instantaneous voltages and currents increase the sputtering yield and excitation/ionization. Harrison and coworkers were the first to apply this technique in mass spectrometric analysis ${ }^{16}$. They even discovered that there were characteristic temporal phenomena for both sputtered and background gas species.

Typically, modulated glow discharge plasmas employ a square wave potential. Current work in this laboratory employs a $25 \%$ duty cycle consisting of $5 \mathrm{~ms}$ of applied power, on time, followed by $15 \mathrm{~ms}$ of no applied power, off time. During the $5 \mathrm{~ms}$ of applied power sputtering and ionization/excitation processes evolve to mimic steady state discharges. In the $15 \mathrm{~ms}$ of power termination that follows, the sample is allowed to cool while sputtered species are removed by the vacuum system. As the applied power reaches a steady state condition, a sharp 
increase in signal can be seen for all background and contaminant gas species. This sharp increase, termed a prepeak, rapidly reaches a maximum within $0.5 \mathrm{~ms}$ of power initiation before decaying to a plateau value. The plateau region for both sputtered and nonsputtered species closely mimics steady state plasma conditions and behavior. Once power has been terminated, certain background species diminish to the baseline value. In contrast, sputtered species show no prepeak behavior, but do show a large maximum, termed an afterpeak, approximately 2-3 ms after power termination. The explanation of prepeak and afterpeak behavior relates to the dominant ionization events that take place throughout the pulse sequence, Figure 1.4.1.1.

When power is initiated, ionization is dominated by electron impact; electrons colliding with and ionizing gas phase species. The absence of ionized sputtered material is explained by the fact that at this time no sputtered neutrals yet exist. lons that sputter away the cathode must first be formed before the sputtering process can begin. This also explains the delay in sputtered ion population. Only atoms and molecules already in the gas phase exhibit prepeak behavior. As more discharge gas ions form and sputter the cathode, the sputtered ion population climbs and reaches a steady state value. Meanwhile the prepeak declines over the next $2 \mathrm{~ms}$ to a steady state value. This decline corresponds with a decrease in the fraction of ionization arising from electron impact. Competing collisional ionization and recombination processes now become significant to the overall chemistry of the plasma. 
At this point and until power termination, the glow discharge mimics steady state conditions. Both sputtered and nonsputtered species have reached a steady state or "plateau" value, for which the time regime is known by. Neutral sputtered species are ionized in the negative glow by electron impact, resonant and nonresonant ionization, and Penning ionization. Once power is terminated, all electron impact ionization ceases as the electrons thermalize. Upon cooling,

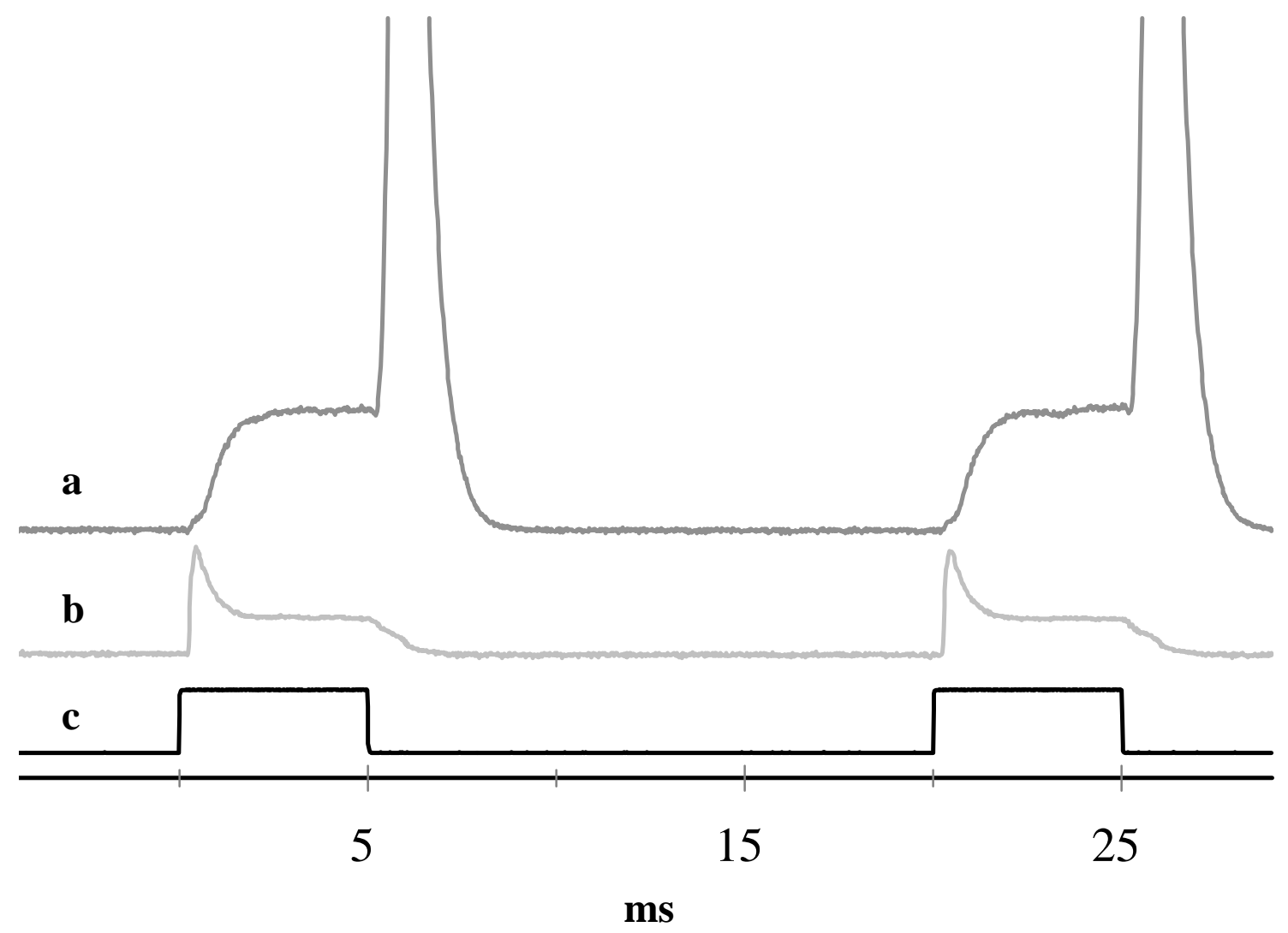

Figure 1.4.1.1 Pulsed profiles of sputtered material(a), the discharge gas argon (b), and the applied power pulse (c).

thermal electrons recombine with discharge gas ions increasing the metastable population. It is significant that the formation of metastable atoms is not an instantaneous process. The time required for higher energy electrons to slow or 
"cool" explains why the afterpeak intensity maximizes .5 - $3 \mathrm{~ms}$ after pulse termination. In the absence of electron ionization, and with this enhancement of metastable atoms, Penning ionization now becomes the dominant ionization process. The metastable energies (11.5 and $11.72 \mathrm{eV}$ ) of argon are too low to ionize neutral argon atoms, water or other contaminant gas species. Only gas phase atoms having ionization potentials below the metastable energy will exhibit afterpeaks. Atomic absorption studies have shown that the metastable argon atom population peaks within $2 \mathrm{~ms}$ of power termination, coinciding with the increase in sputtered ion population. ${ }^{12}$

The analytical utility of modulated plasmas with time-gated detection to exploit enhanced Penning ionization provides increased sensitivity. Such methods use gated detection in selected regions of temporal profiles to capture analytical signals at their maximum while background signals are at their minimum ${ }^{13}$. Recently, modulated plasma research has been extended to the application of microsecond pulsed plasmas ${ }^{14}$. Because microsecond plasmas can achieve even higher instantaneous powers and greater duty cycles, they should provide greater degrees of sputtering, excitation and ionization. 


\section{Instrumentation}

Glow discharges reached greatest analytical utility in the late 1960 s as

emission sources for trace elemental determination of solid samples ${ }^{15}$. The universal utility of the glow discharge as both an emission and ion source has been demonstrated on a variety of optical mass and spectrometers instruments. Modulated or pulsed plasmas have been shown to have analytical utility when used with quadrupole ${ }^{16}$, ion trap, ${ }^{17}$ and time of flight instruments. ${ }^{18,19}$

\subsection{Pulsed Glow Discharge lon Source}

The glow discharge source consists of a discharge chamber, a direct insertion probe (DIP) and a sample introduction chamber. The simplicity of its design facilitates its versatility and ease of coupling to different mass analyzers or optical systems. The discharge chamber itself is a six-way high vacuum stainless steel cross (MDC, Hayward, CA). Side ports on the cross are equipped with a probe inlet, ion exit orifice, and gas inlet and outlet. The remaining ports are fitted with stainless steel conflat flanges or suprasil optical view ports (Herareus Quartz, Duluth, GA). The probe inlet consisted of a ball valve fitted with a $1 / 2$ " adapter that allowed direct probe insertion without breaking the system vacuum, Figure 2.1.1.1. The ion exit orifice used in mass spectrometric measurements was positioned $180^{\circ}$ with respect to the probe inlet, while view ports were positioned orthogonally for optical monitoring. Pressure in the discharge chamber was monitored through one of the gas outlets by a thermocouple pressure gauge (Teledyne-Hastings, Hampton, VA). 


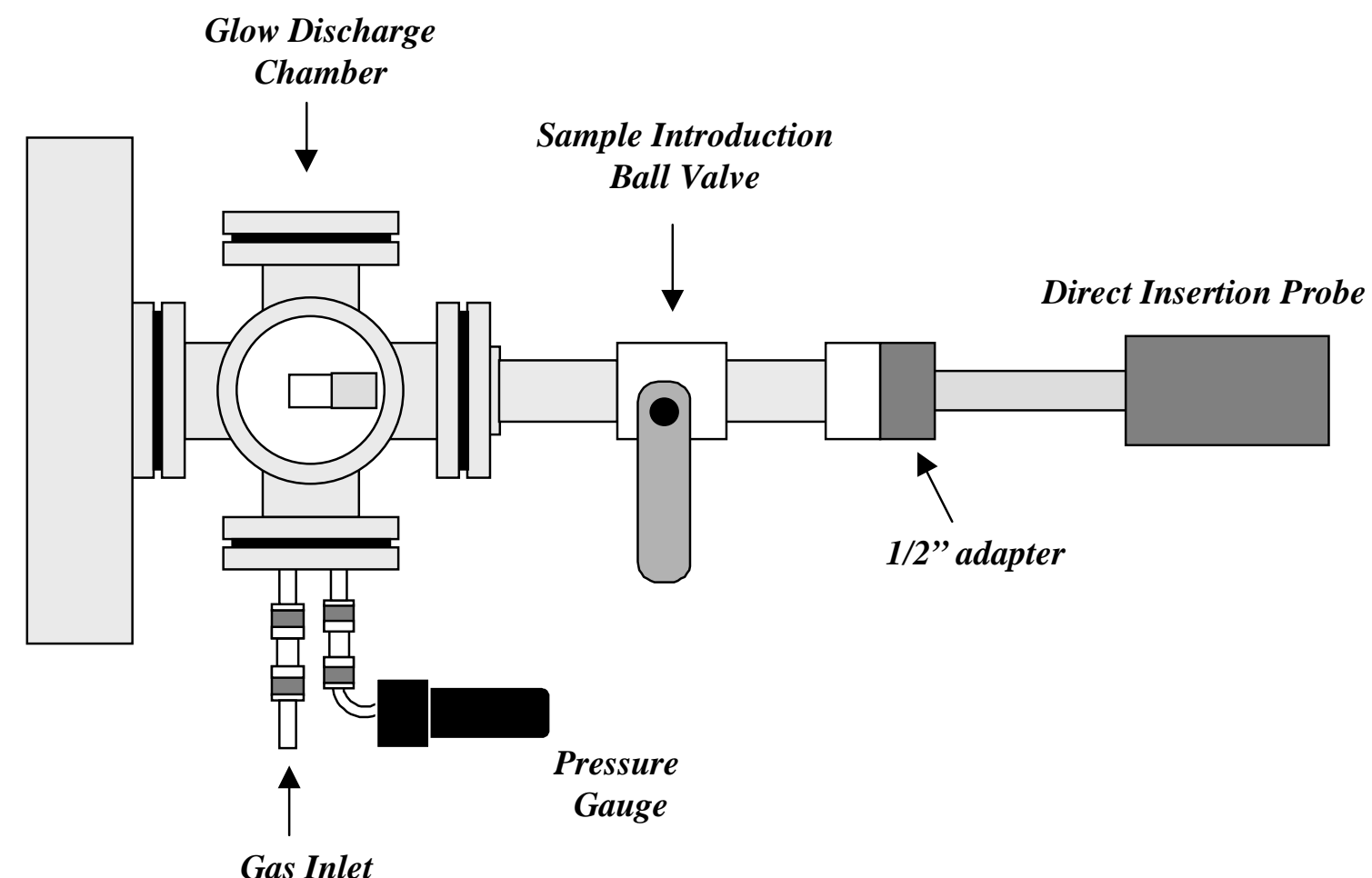

Figure 2.1.1.1 The glow discharge ion source

Direct insertion probes (DIP) differ in design according to the potential which they carry; rf or dc. The rf's ability to flow through nonconducting samples may be advantageous for certain analyses, but it poses certain insulating challenges over its dc counterpart.

The dc probe body was constructed from a $1 / 2$ " stainless steel tube, shown in Figure 2.1.1.2. A copper wire running the length of the probe was silver soldered to a high-pressure feedthrough and MHV connector located at each end. A silicone coated woven fiberglass tube insulated the internal wire and feedthrough from the probe body. The rf probe required an N-type connector to supply power to the probe. Additional modifications include the replacement of the copper wire 
with an oxygen free copper rod surrounded by glass tubing to provide insulation.

It is critical that dead spaces and shielding are employed to prevent rf leakage.

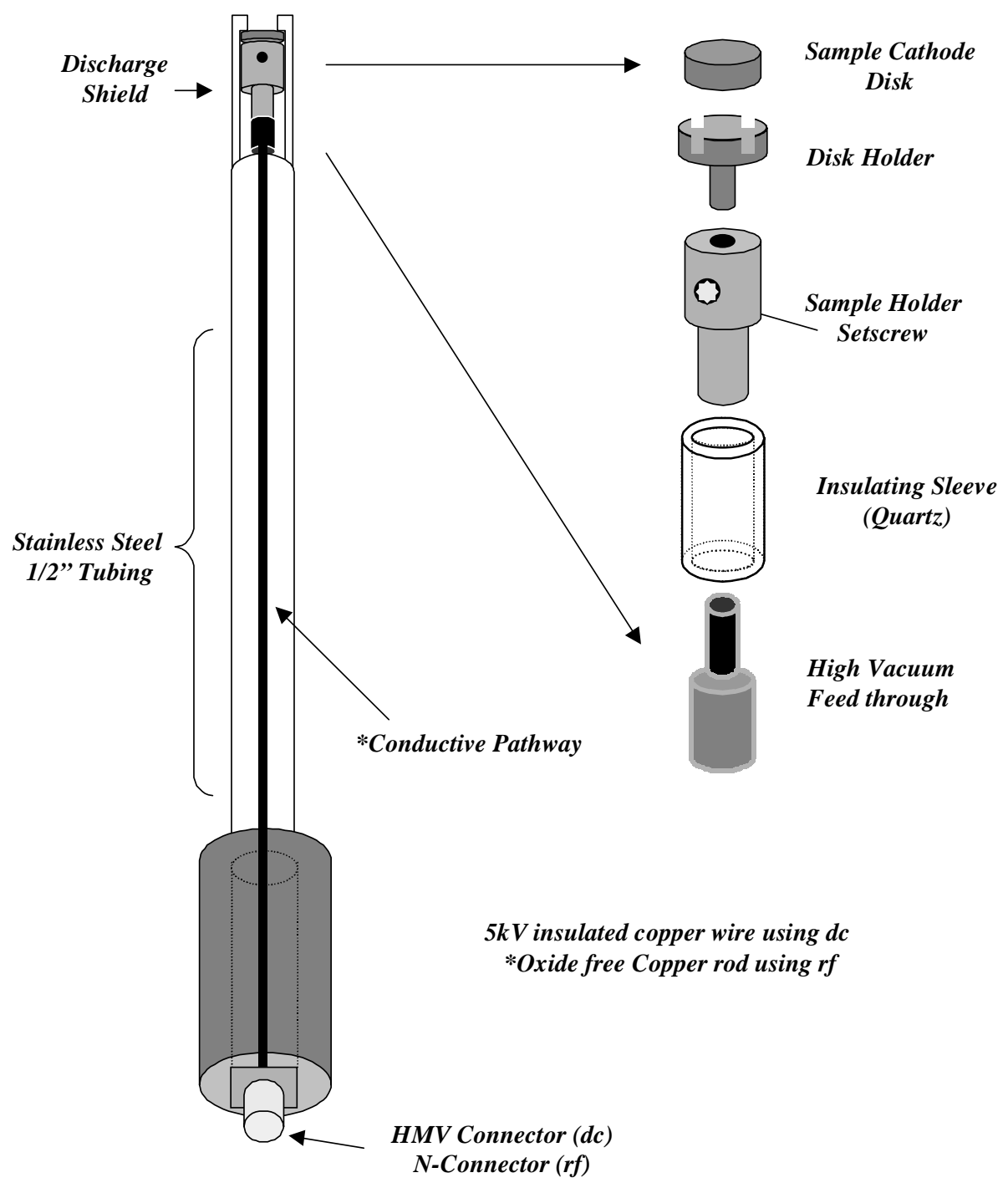

Figure 2.1.1.2 Construction of the dc direct insertion probe (DIP) and the rf probes differ in the conductive pathway. Also the rf DIP uses insulating glass sleeves inserted around the copper rod (not pictured). 
Glow discharge samples are either machined or pressed into the traditional pin or disk geometry. They are then placed in the mounting assembly on the end of the DIP probe. The mounting assembly consisted of a copper connector that was placed over the center of the probe feedthrough. A small quartz tube was placed at the base of the connector to prevent the connector from arcing. The assembly is tapped with a setscrew holding the pin or sample disk holder in place. A machined ceramic jacket is placed over and around the mounting assembly to shield against the discharge. When using an rf potential the ceramic jacket was wrapped in aluminum foil to provide a ground to the probe body and prevent the ceramic jacket from being sputtered.

The dc glow discharge was powered by a fast operational power supply (OPS-3500, Kepco, Flushing, NY). The power supply's output was driven and controlled by a pulse generator that allowed for control of the applied power level, frequency, and duty cycle. Radio frequency power was supplied by a $13.56 \mathrm{MHz}$ rf generator (Model RF 10-S, RF Plasma Products, Inc., Marlton, NJ) and coupled through an automatic matching network (Model AM-10, RF Plasma Products Inc., Marlton, NJ). The internal pulsing mode of the generator permitted control of the duty cycle, frequency and applied power. 


\section{Spectral, Spatial, and Temporal Characterization of a Millisecond Pulsed Glow Discharge: Optical Characterization}

\subsection{Introduction}

Pulsed operation of the analytical glow discharge source affords unique analytical advantages as well as alleviating plasma instability arising from resistive overheating of the sample cathode. ${ }^{20}$ Such operation of pulsed glow discharge sources consists of an applied square wave power pulse followed by a period of power termination. The width of the applied power pulse may vary from several microseconds $(\mu \mathrm{s})$ to milliseconds $(\mathrm{ms})$ at frequencies up to $100 \mathrm{~Hz} .^{21,12}$ A principal analytical advantage is the creation of time regimes in which analyte signal is enhanced and background signal is suppressed. Time gated detection permits this advantage to be exploited.

Illustrations of the analytical utility of pulsed glow discharge sources coupled with time-gated mass spectrometric detection include; 1) the temporal resolution between signals of ions arising from the discharge working gases and that from sputtered analyte ${ }^{16,22}$; and 2) the determination of molecular weight and structure of organic molecules. ${ }^{23}$ These examples rely on the temporal characteristics of the dynamic gas phase reactions that occur in these pulsed glow discharge sources. The current work shows that further advantages may be gained from exploitation of the spatial characteristics of these plasmas as well.

A typical ms-pulsed glow discharge operating sequence includes an applied power pulse of $5 \mathrm{~ms}$ followed by power termination for $15 \mathrm{~ms}$. During the 
$5 \mathrm{~ms}$ applied power pulse, gas phase species and sputtered species undergo a series of excitation and ionization processes. Of these processes, electron excitation and Penning ionization are dominant. ${ }^{9}$ Upon power termination, the electron excitation process halts, as the plasma changes from an excitation/ionization mode to a recombination mode. In this recombination mode, thermal electrons and plasma ions recombine to produce excited and metastable atoms. At this time in the pulse cycle, the enhancement of the metastable argon atom population leads to an increase in Penning ionization. ${ }^{10}$ This enhancement of Penning ionization not only leads to an increase in ionization of sputtered atoms but also leads to an increase in emission from the sputtered atoms. Because of these enhancements in analyte signals, this "afterpeak" time period is of great interest to the analyst. ${ }^{20}$

This chapter describes a series of optical investigations of ms-pulsed glow discharge species designed to elucidate processes throughout the pulse cycle. This is accomplished by monitoring emission and absorbance from several argon bath gas and sputtered analyte transitions. The differences in excitation and ionization mechanisms of these species and their spatial as well as temporal separation are the focus of the studies reported here.

\subsection{Experimental}

Detailed descriptions of the glow discharge source and optical spectrometry system employed here appear in previous paper. ${ }^{12}$ The glow discharge operated at a pulse rate of $50 \mathrm{~Hz}$ with a $25 \%$ duty cycle and an instantaneous applied power level of 1.5 watts. The operating pressure was 
maintained at 0.8 Torr with ultra pure argon gas (Airgas, Radnor, PA). The glow discharge sample cathode consisted of a $4 \mathrm{~mm}$ diameter copper disk (SRM 495, NIST Gaithersburg MD) that was mounted on the end of a direct insertion probe. Vertical positioning was obtained through careful adjustment of the direct insertion probe with respect to the center of the discharge chamber; whereas, horizontal positioning was obtained by use of an X-Y positioning stage on which the glow chamber was mounted.

Transient emission signals were recorded directly from the photomultiplier tube (Model R-928, Hamamatsu, Japan) by a 1-GHz oscilloscope (9370M, LeCroy, Chestnut Ridge, NY). Each digitized trace represented the summation of 100 discharge pulse cycles, each consisting of 2500 data points. Atomic absorbance measurements were collected using a copper-neon and boron-argon hollow cathode lamps operated at $16 \mathrm{~mA}$. The lamp output was modulated at 3 kHz by a mechanical chopper (197, EG\&G PAR, Princeton, NJ). The chopped beam was then focused through the glow discharge cell and plasma onto the entrance slit of the 0.64 m monochromator (ISA HR-640, Edison, NJ). Both the entrance and exit slits were fixed at $50 \mu \mathrm{m}$. Signal response from the photomultiplier tube was processed by a lock-in-amplifier (5210, EG\&G PAR, Princeton, NJ) locked to the chopper frequency. An oscilloscope was used to monitor the temporal output from the LIA and to digitize the resulting data.

Absorption measurements of the ground state copper atom and metastable argon atom populations were accomplished by monitoring the absorption of $324.75 \mathrm{~nm}$ and $811.53 \mathrm{~nm}$ emission from the appropriate hollow 
cathode lamp. Deconvolution of the $1 \mathrm{~ms}$ LIA time constant was accomplished, using a low pass filter approximation and a series of Fourier transforms, inside of a spreadsheet program (Excel, Microsoft, Seattle, WA) ${ }^{24}$ Following deconvolution, the data were baseline corrected and smoothed by repetitive moving averages of 10 points. The resultant digitized emission and deconvoluted absorbance traces were used to construct spatial maps of the glow discharge plasma consisting of such measurements collected at 368 spatial positions throughout the plasma, Figure 3.2.1.1. 


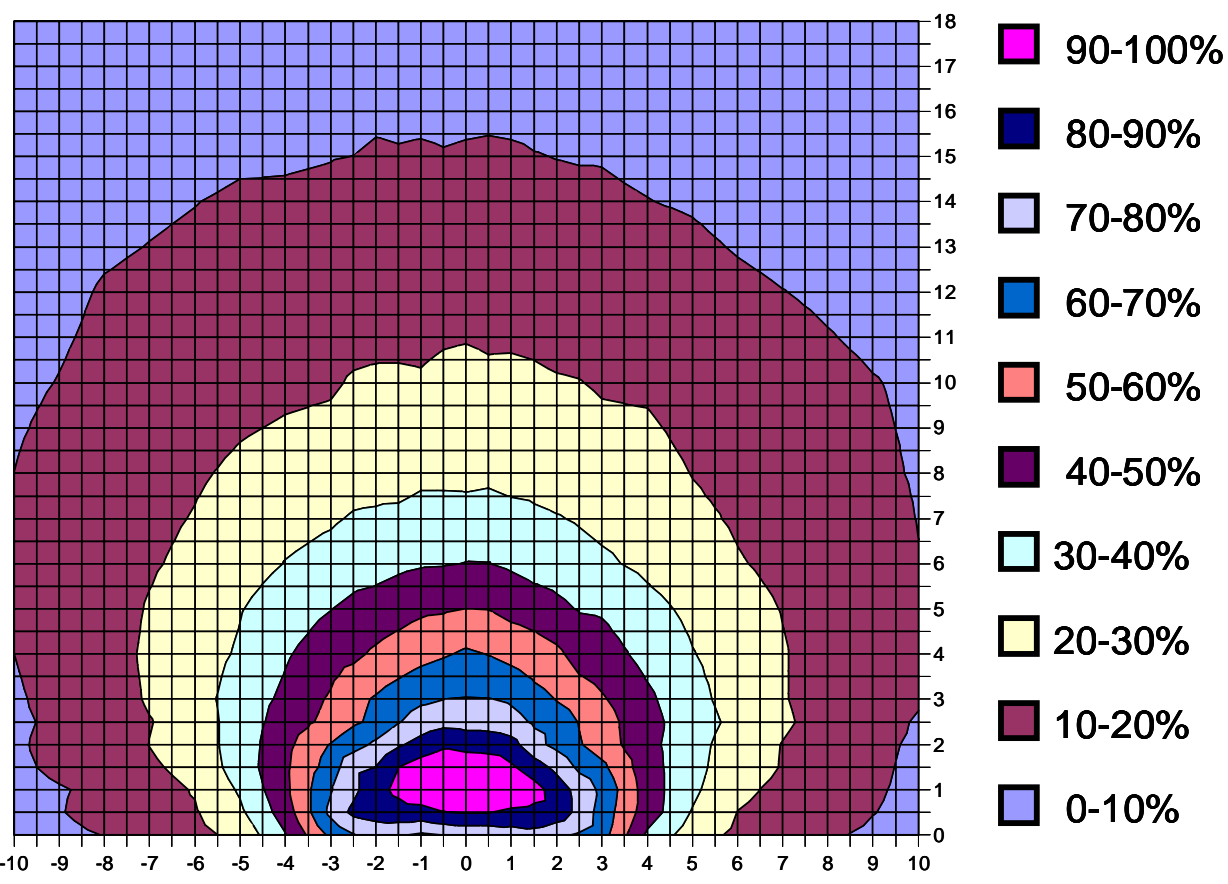

\section{Observation Points}

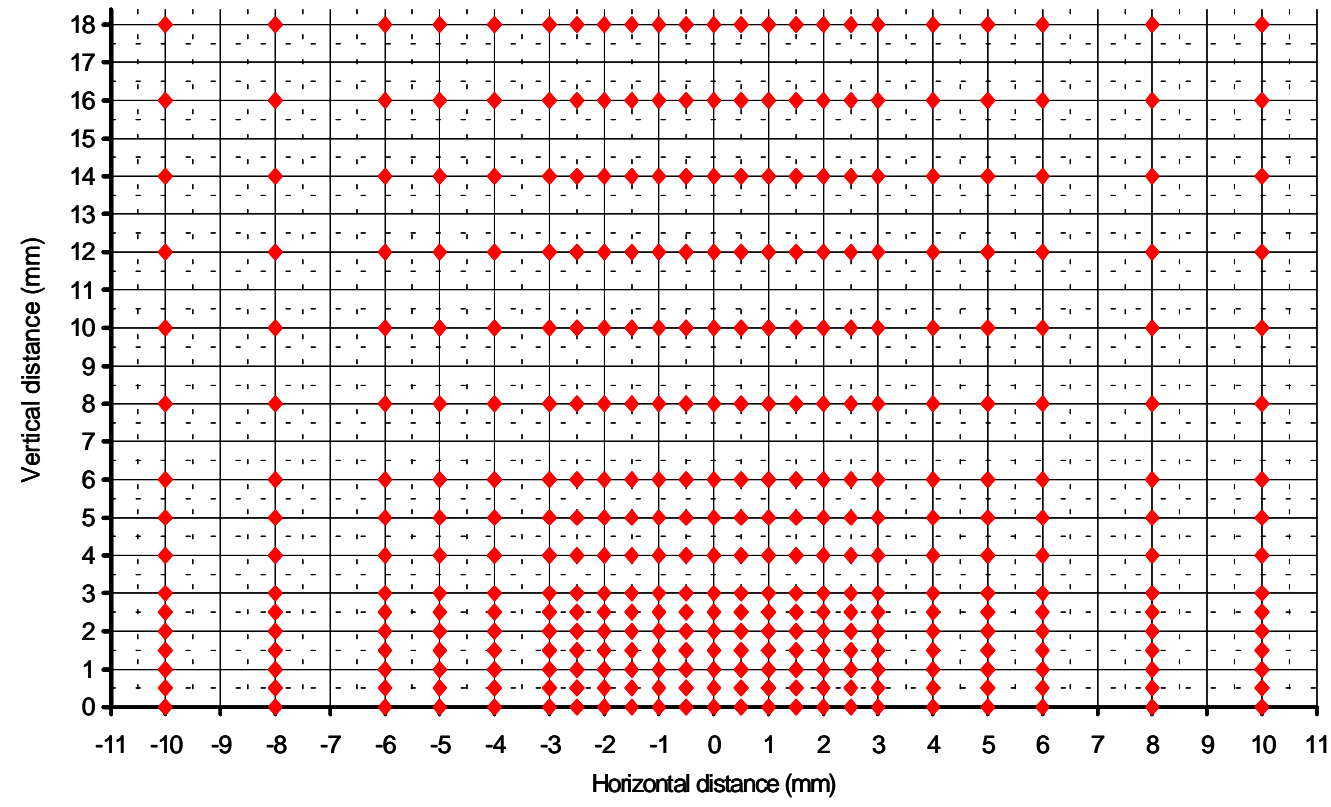

Figure 3.3.2.1 Emission and Absorbtion maps key read in units of $10 \%$ of maxmium intensity, while aborbance units are in $10 \%$ of maxim absorbance values. The lower diagram depicts where the 368 measurements were made. 


\subsection{Results and Discussion}

\subsubsection{Argon Ion Emission}

A series of argon ion emission lines were monitored at increasing distance from the cathode into the negative glow, Table 3-1. In general, argon ion emission was found to maximize 2.0 to $3.0 \mathrm{~ms}$ into the pulse cycle at a distance of $2 \mathrm{~mm}$ from the cathode surface, Figure 3.3.1.1. Each emission profile exhibited emission only during the plateau time regime, that is none exhibited a prepeak or afterpeak (Figure 3.3.1.2). This observation is consistent with previous pulsed glow discharge studies. The most probable mechanism of argon ion emission would be a two-step process involving ionization followed by electron excitation. The absence of the characteristic prepeak reported in previous mass spectrometric studies of ${ }^{40} \mathrm{Ar}^{+}$supports this mechanism. The absence of argon ion emission once power was terminated arises because of the high-energy electron population needed for excitation has decayed.

Temporal argon ion emission maps, Figure 3.3.1.3, were constructed by monitoring emission, at $476.4 \mathrm{~nm}$, corresponding to an electronic transition 19.76 $\mathrm{eV} \rightarrow 17.26 \mathrm{eV}$ above the argon ion ground state. These maps demonstrate that argon ion emission is localized around the cathode surface and radially decays as distance increases. Emission intensity reaches a plateau value within $3 \mathrm{~ms}$ of the glow discharge initation, this steady state continues until 0.10 ms past power termination. Upon power termination, argon ion emission decays without any noticeable diffusion. 


\begin{tabular}{|c|c|c|c|c|}
\hline \multirow{2}{*}{ Wavelength } & \multicolumn{2}{|c|}{$\begin{array}{c}\text { Glow Discharge Intensity } \\
\text { Monochramtor }\end{array}$} & \multicolumn{2}{c|}{$\begin{array}{c}\text { Energy } \\
\text { Levels } \mathbf{~ m s}\end{array}$} \\
\hline $\mathbf{n m}$ & Counts & $\mathbf{m V}$ & $\mathbf{E}_{\mathbf{L}} \mathbf{~ e V}$ & $\mathbf{E}_{\mathbf{H}} \mathbf{e V}$ \\
\hline 413.173 & 21540 & 113 & 18.43 & 21.43 \\
\hline 427.752 & 39010 & 227 & 18.45 & 21.35 \\
\hline 433.120 & 4840 & 40 & 16.75 & 19.61 \\
\hline 434.806 & 23310 & 110 & 16.64 & 19.49 \\
\hline 437.075 & 11130 & 66 & 18.66 & 21.49 \\
\hline 442.601 & 17445 & 94 & 16.75 & 19.55 \\
\hline 454.505 & 32635 & 189 & 17.14 & 19.87 \\
\hline 457.935 & 14080 & 81 & 17.26 & 19.97 \\
\hline 458.990 & 22355 & 133 & 18.43 & 21.13 \\
\hline 460.956 & 41600 & 221 & 18.45 & 21.14 \\
\hline 465.789 & 28070 & 158 & 17.14 & 19.80 \\
\hline 472.686 & 22900 & 129 & 17.14 & 19.76 \\
\hline 476.486 & 41630 & 280 & 17.26 & 19.87 \\
\hline 480.602 & 15215 & 80 & 16.64 & 19.22 \\
\hline 487.986 & 23905 & 152 & 17.14 & 19.68 \\
\hline 496.507 & 10045 & 61 & 17.26 & 19.76 \\
\hline
\end{tabular}

Table 3-1 Argon ion emission intensities taken at $5.0 \mathrm{~ms}$ into the pulse cycle at $2.0 \mathrm{~mm}$ from the cathode surface with the corresponding transitions high $\left(\mathrm{E}_{\mathrm{H}}\right)$ and low $\left(\mathrm{E}_{\mathrm{L}}\right)$ energy levels above the argon ion ground state. 


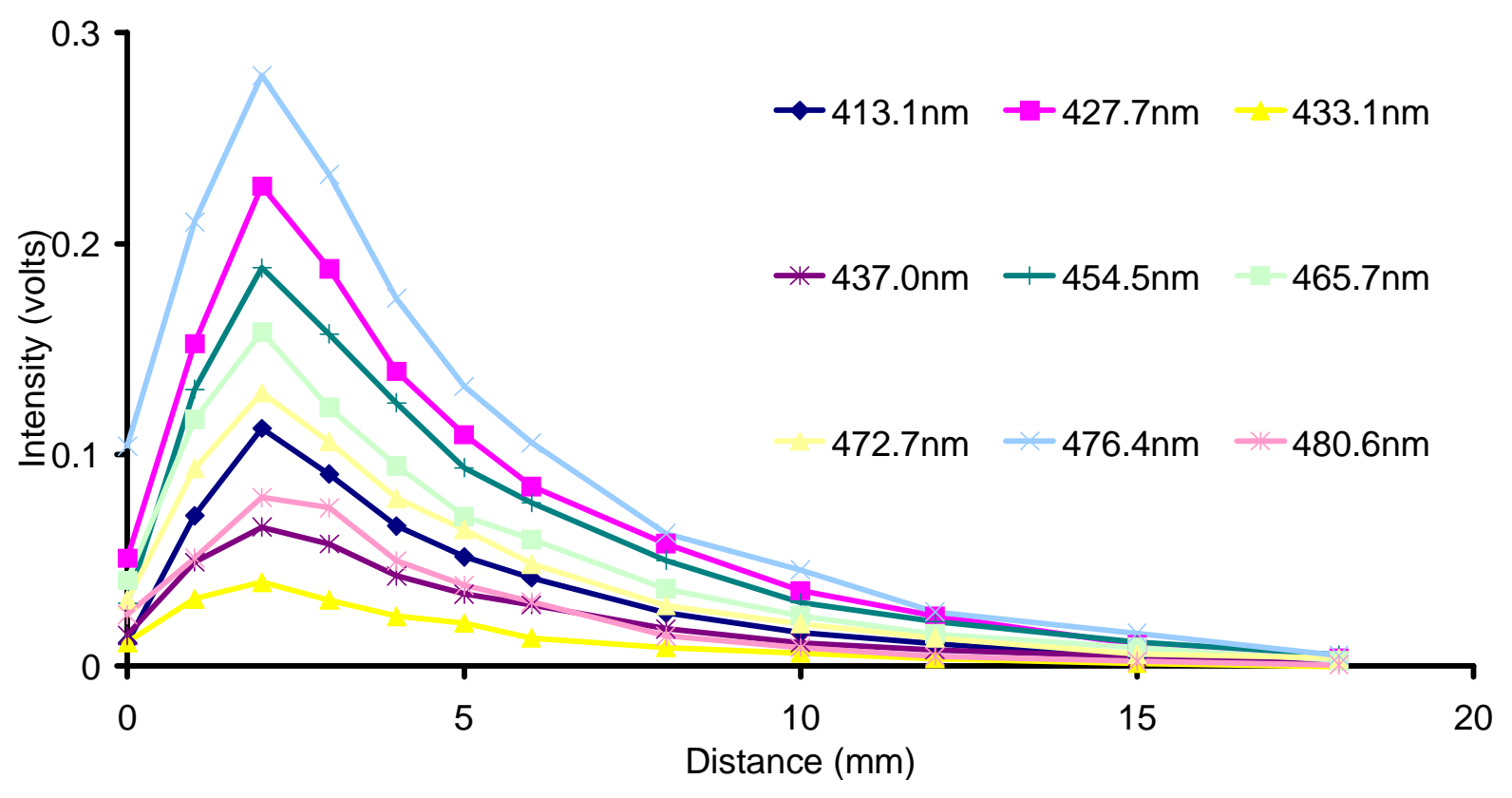

Figure 3.3.1.1 Two dimensional plots of argon ion emission monitored at different wavelengths as a function of cathode distance.

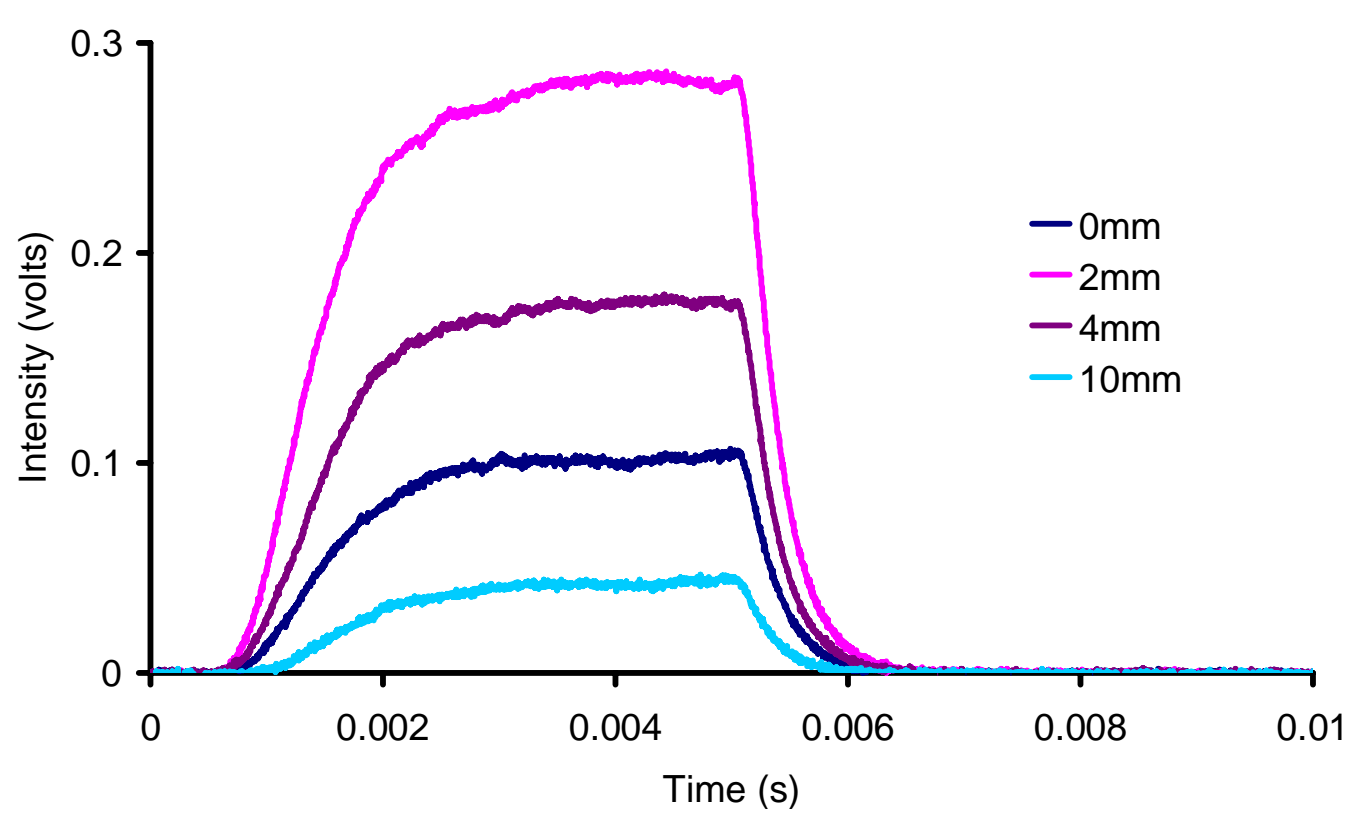

Figure 3.3.1.2 The temporal emission profiles of argon ion, monitored at $476.4 \mathrm{~nm}$ as a function of increasing distance from the cathode surface. 

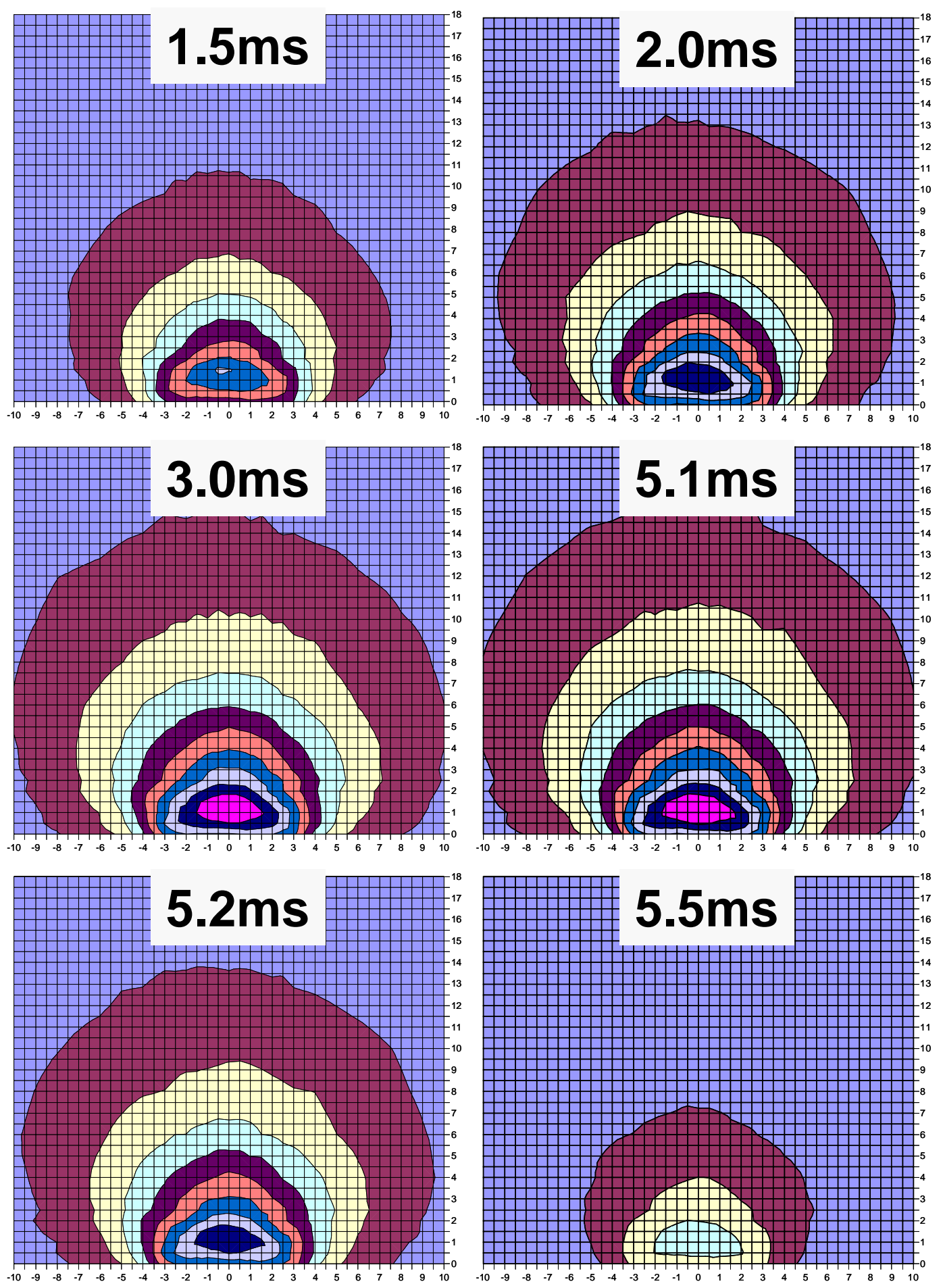

Figure 3.3.1.3 Two-dimensional maps of argon ion emission monitored at $476.4 \mathrm{~nm}$ throughout the pulse cycle. 


\subsubsection{Metastable Argon Atoms}

The significant role of the metastable argon atom species has been discussed previously as the source of Penning ionization. The metastable argon atom population was investigated by monitoring the ${ }^{3} \mathrm{P}_{2}$ state $(11.52 \mathrm{eV})$ at a wavelength of $811.5 \mathrm{~nm}$ using atomic absorption and emission measurements. This emission corresponds to radiative decay of the ${ }^{3} \mathrm{D}_{3}-{ }^{3} \mathrm{P}_{2}$ states occurring 13.08-11.55 eV above the atom ground state. Both emission and absorption temporal profiles demonstrate a large discrete maximum within $2 \mathrm{~mm}$ of the cathode surface, Figures 3.3.2.1 and 3.3.2.2. This maximum occurs during the plateau time regime, and at this distance no afterpeak is observed. Monitoring of both emission and absorption at increasing spatial distance demonstrates a decline in the large plateau population, and the subsequent formation of an afterpeak that maximizes 5 to $8 \mathrm{~mm}$ from the cathode surface. This different spatial behavior can be explained by considering the two different mechanisms of metastable production, i.e. excitation and recombination.

Close to the cathode surface metastable production is primarily through collisional excitation involving fast argon ions and atoms. ${ }^{25}$ At this distance, recombination of argon ions and electrons is considered to have a low probability. This is due to the low population of thermalized electrons in this area, because of the high electric field strength. Metastable production through recombination will occur in the region of greatest overlap between thermal electrons and argon ions. Such processes would be favored at greater distances, where the absence of cathode electronic field strength would allow for 
the thermalization of the electron population. This would lead to the conclusion that the afterpeak observed in these pulsed plasmas is predominately through recombination events. Both emission and absorption profiles demonstrate an apparent afterpeak within $8 \mathrm{~mm}$ of the cathode surface. The absorption and emission data collected suggest that this afterpeak population is only a fraction to half of the temporal maximum of the argon metastable population found throughout the pulse cycle.

Once formed, these metastable argon atoms can only release their potential energy $(11.52 \mathrm{eV})$ through collisions with other plasma species or through photon or electron de-excitation. The temporal position of this maximum occurs ca. 0.3 to $0.5 \mathrm{~ms}$ after power termination, and is the dominant ionization processes at this time. As will be discussed in the next section, this spatial and temporal population overlaps with the emission of certain analyte transitions suggesting a direct correlation between the two species. It is important to note, however, that even though the larger majority of the metastable population occurs close to the cathode surface, no appreciable afterpeak for the metastable population or sputtered species appears at this distance. This suggest that at close cathode distances the metastable population does not lead to sputtered atom ionization and is most likely de-excited by the high electron population in this area. 

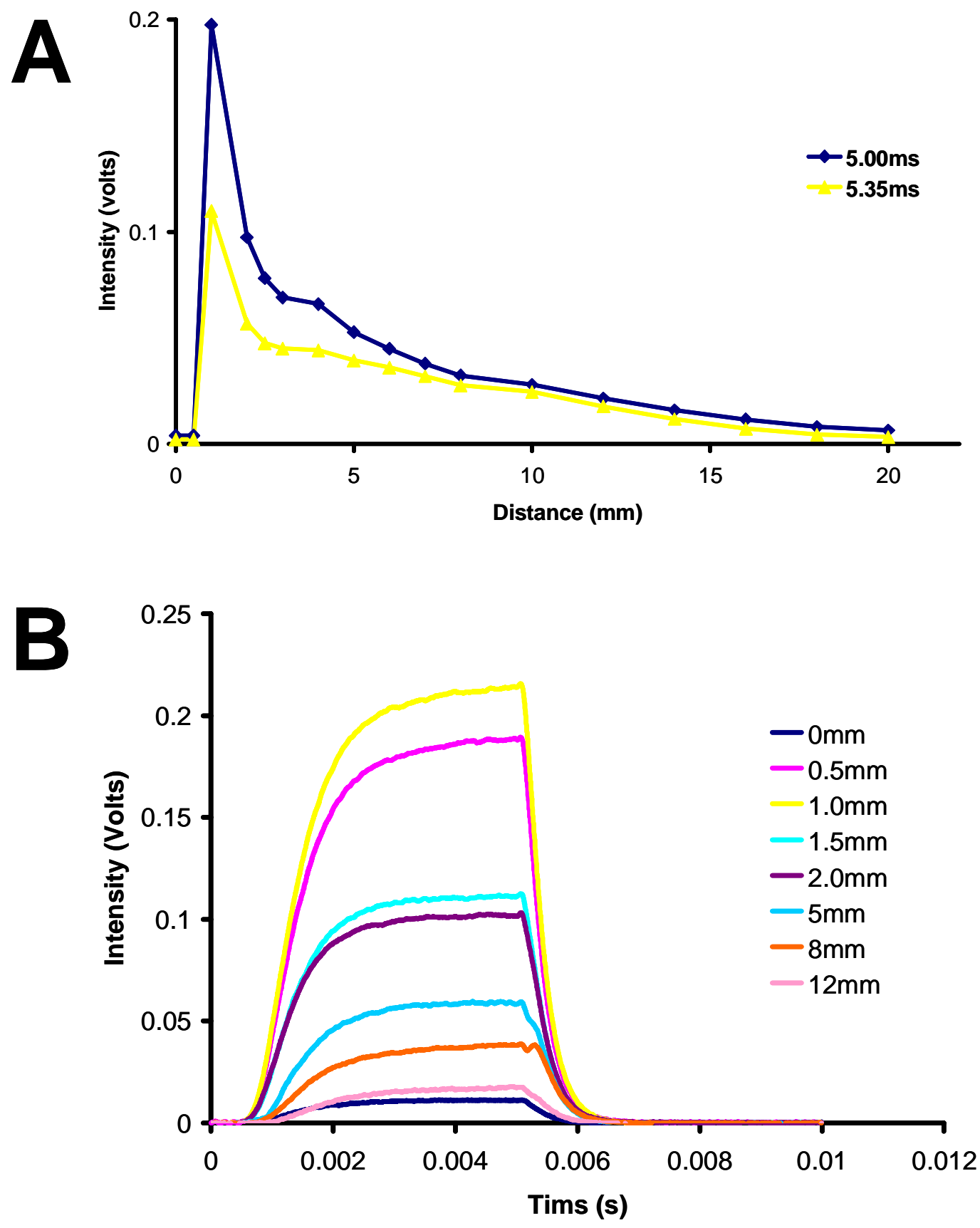

Figure 3.3.2.1 (A) The emission intensity as a function of distance during the plateau and afterpeak time regimes. (B) The temporal emission profiles, monitored at $811.5 \mathrm{~nm}$. 


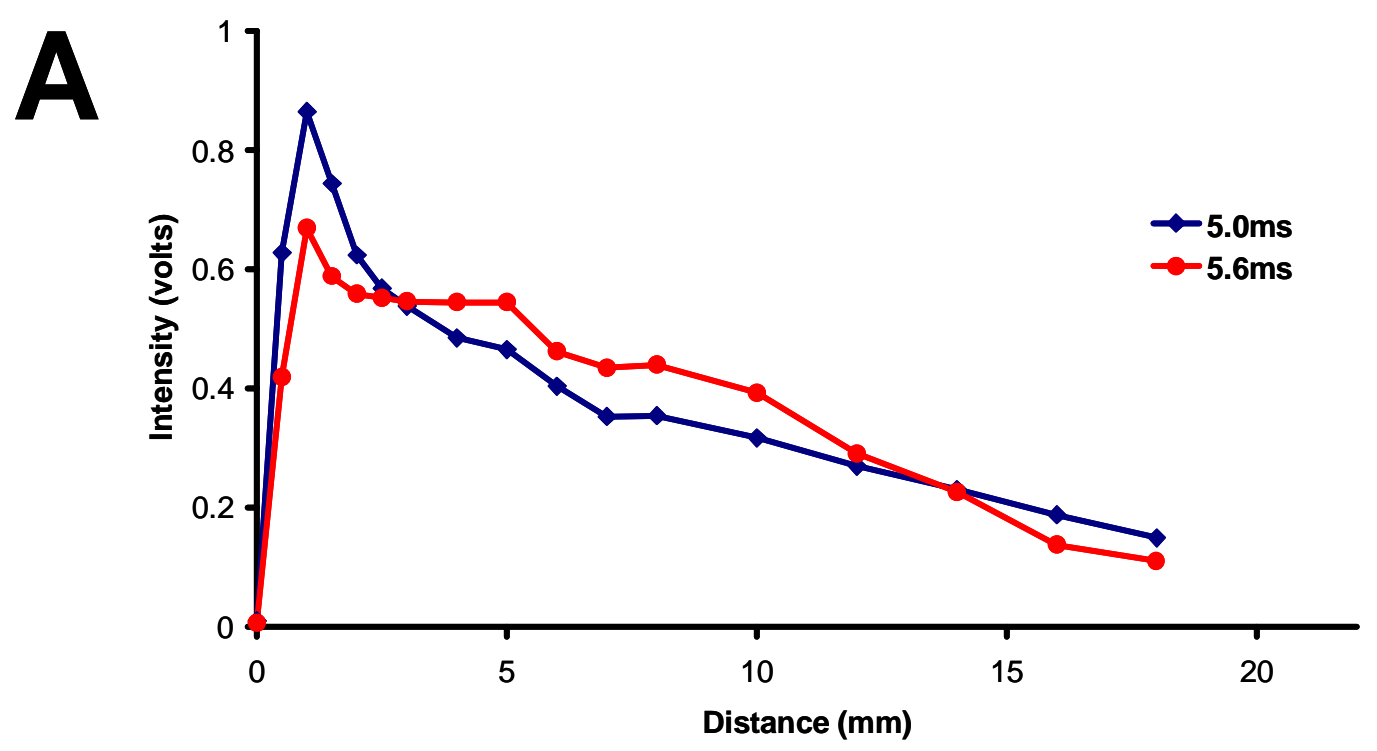

B

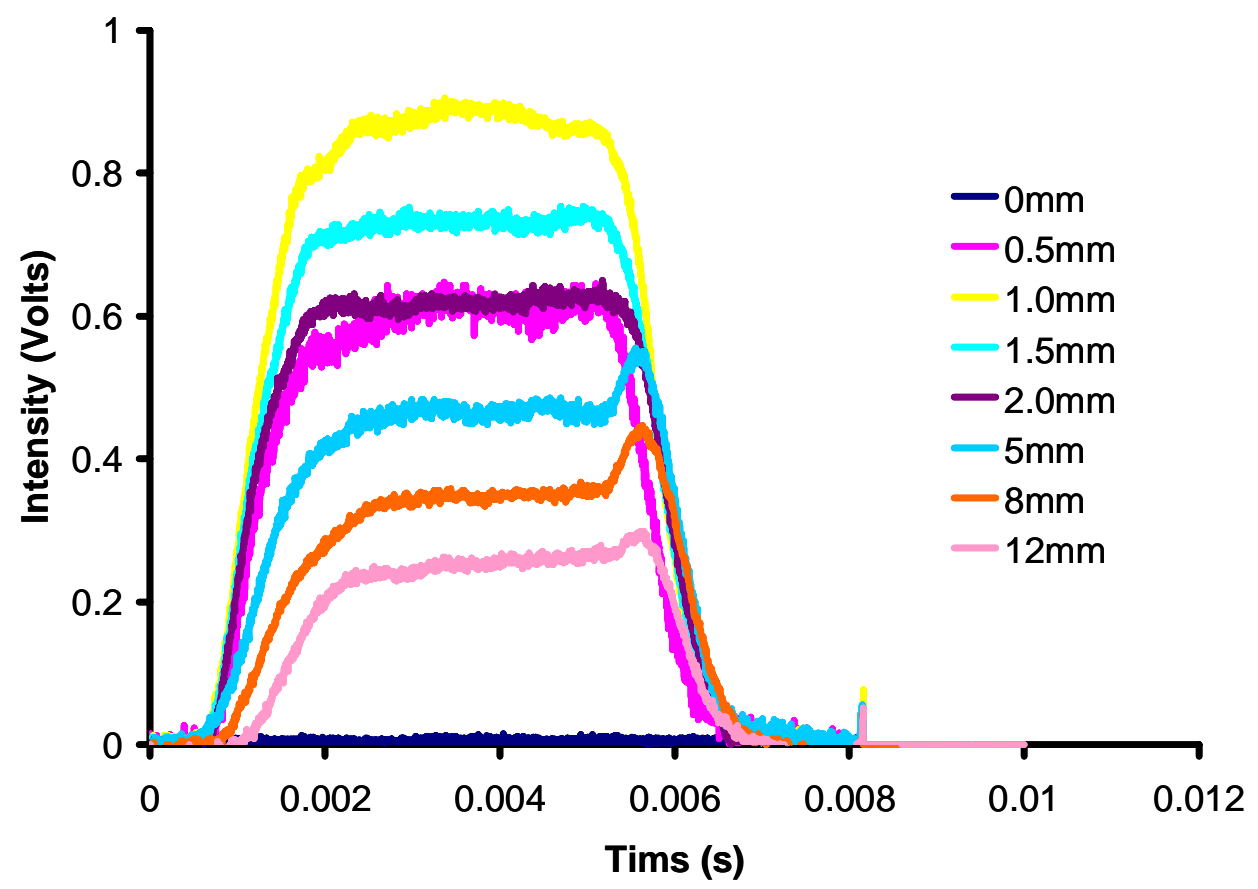

Figure 3.3.2.2 (A) The above plot illustrates the absorption of the metastable argon atoms as a function of distance during the plateau and afterpeak time regimes. (B)The temporal absorption profiles of the argon metastable, monitored at $811.5 \mathrm{~nm}$, at increasing distances. 
Two-dimensional emission and absorption maps, Figures 3.3.2.3 and 3.3.2.4, were constructed to demonstrate the relative changes in the metastable population throughout the pulse cycle. Both maps clearly show this discrete spatial maximum occurring approximately $1.5 \mathrm{~mm}$ from the cathode surface. This population reaches steady state conditions at $3.0 \mathrm{~ms}$ into the pulse cycle and remains until $5.1 \mathrm{~ms}$ past power termination for the emission maps, and until $5.5 \mathrm{~ms}$ for the absorption maps. Even though the absorption maps have been deconvouted, there still appears to be a slight shift in temporal position. Barring this discrepancy in absorption maps temporal position; both maps clearly show a shift in position of the metastable population after power termination. This spatial position occurs 5 - $8 \mathrm{~mm}$ from the cathode surface. For emission maps this maximum occurs between 5.3 to $5.5 \mathrm{~ms}$, at an intensity value of $20-30 \%$ of the emission temporal maximum. For the absorption maps this maximum occurs at $5.9 \mathrm{~ms}$ into the pulse cycle at $60-70 \%$ of the absorption pulse cycle temporal maximum. Once formed both emission and absorption maps show no particular diffusional behavior as it decays over the next 1 to $2 \mathrm{~ms}$. 

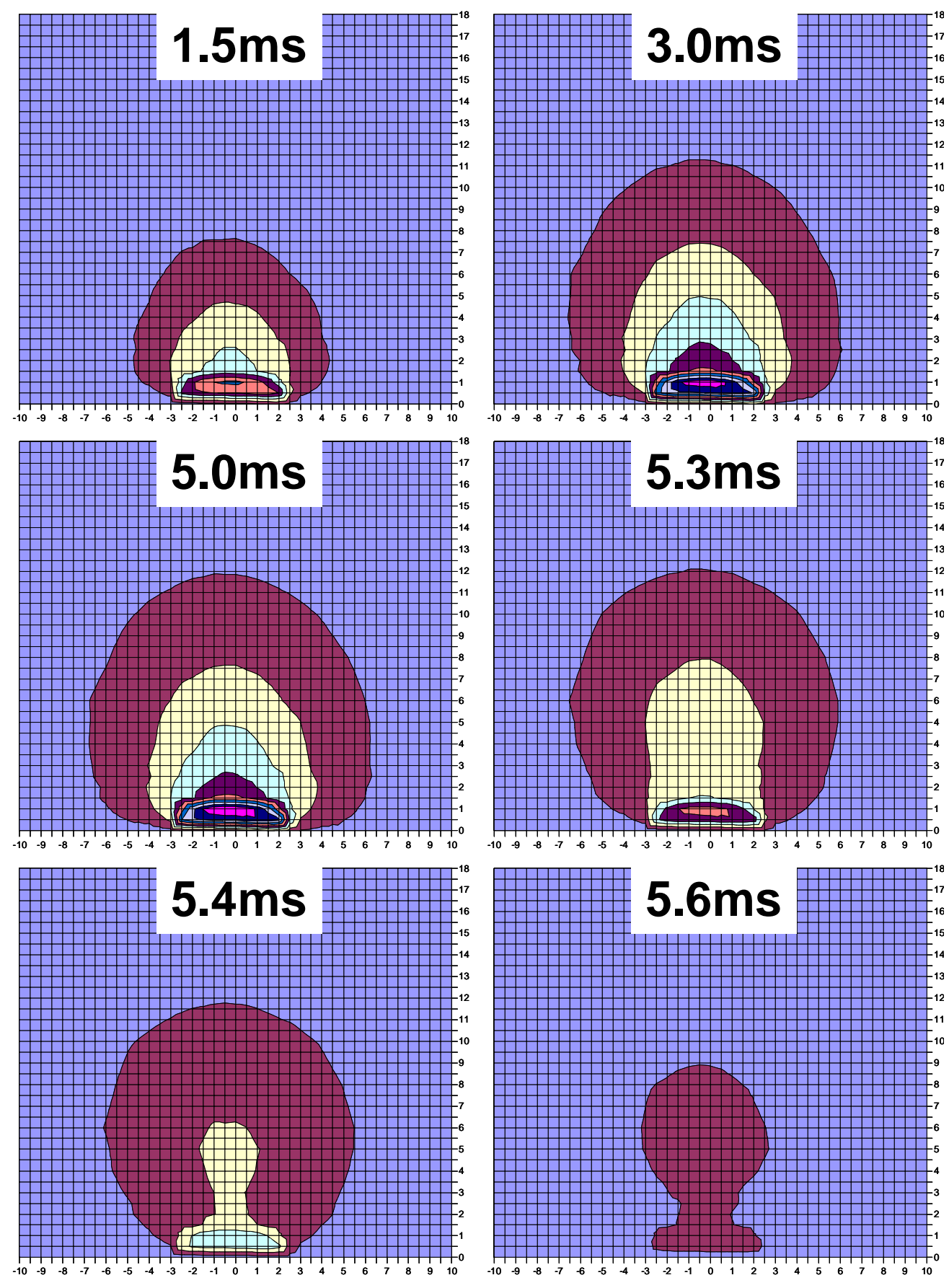

Figure 3.3.2.3 Two-dimensional emission maps of the formation of metastable argon atoms, ${ }^{3} \mathrm{P}_{2}$, monitored at $811.5 \mathrm{~nm}$. 

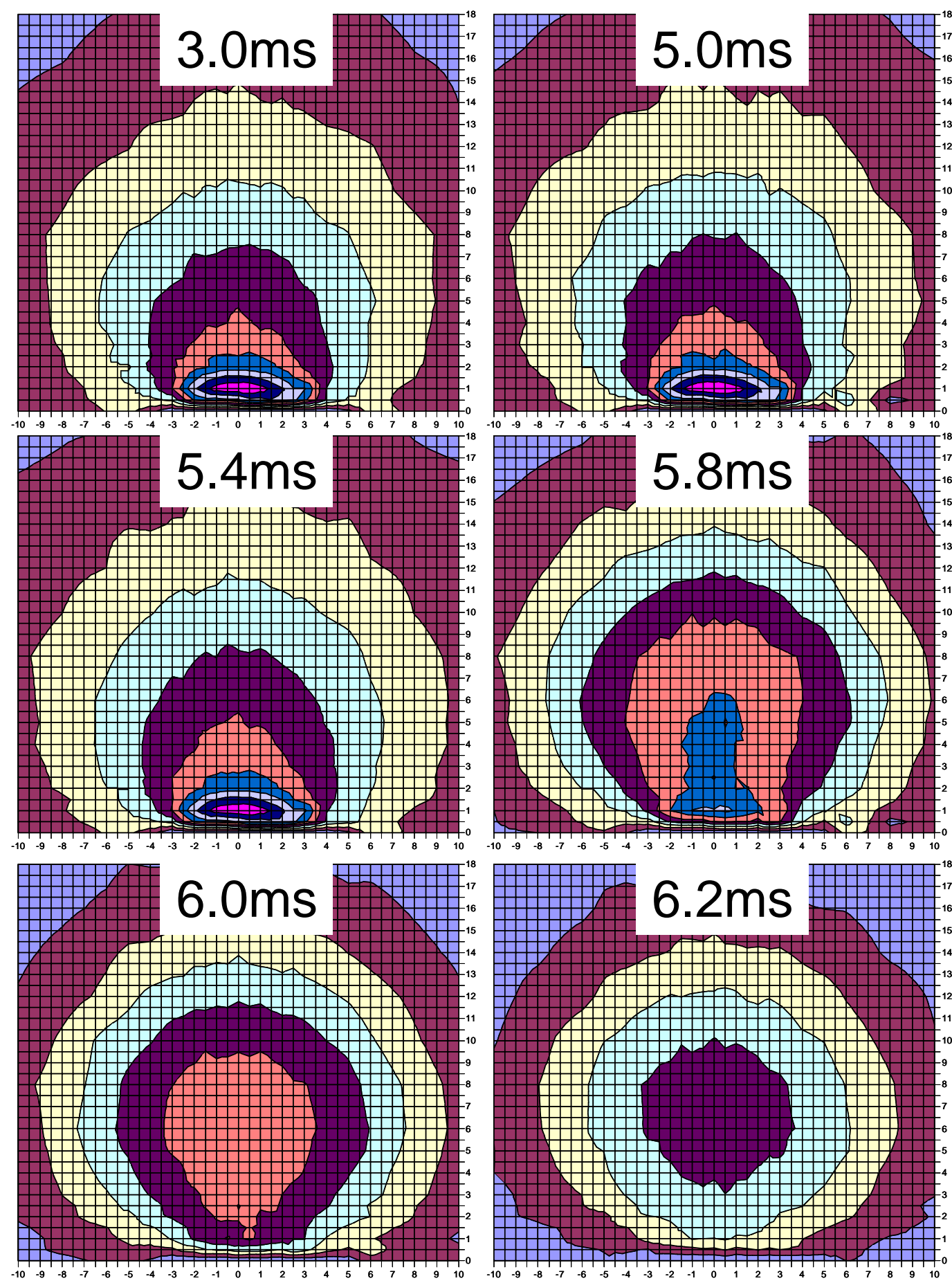

Figure 3.3.2.4 Two dimensional absorbance maps of the metastable argon atoms population monitored through out the pulse cycle using hollow cathode emission at $811.5 \mathrm{~nm}$. 


\subsubsection{Sputtered Analyte Emission: Copper Atom Transitions}

These studies examine the temporal behavior for a series of copper atom transitions at increasing spatial distances from the cathode in the negative glow. Each series of electronic transitions demonstrates the characteristic plateau and afterpeak behavior. In general, plateau intensities maximize 2.5 to $3.0 \mathrm{~ms}$ into the pulse cycle. At this time, and until power termination, the glow discharge exhibits a steady state behavior as if it were not being pulsed. This steady state behavior indicates the establishment of equilibrium between excitation, deexcitation, and recombination processes. When power is terminated, plasma processes are dominated by the recombination of analyte and discharge gas ions with thermalized electrons. The result of these recombination processes is the formation of excited or metastable atoms for both the analyte and discharge gas species. Thus, as stated previously, each copper atom transition exhibits an afterpeak, which is a surge in emission intensity ca. $0.3-0.6 \mathrm{~ms}$ after power termination. Because electron excitation is quenched at the end of the power pulse, the source of this emission is recombination of copper ions and electrons followed by radiative decay. Experimental data obtained for various electronic transitions for both plateau and afterpeak emission intensities are summarized in Table 3-2. This table, constructed using emission data taken at $6.0 \mathrm{~mm}$ from the cathode surface, is presented to be both illustrative and informative of typical results. The listed plateau intensity values in Table 3-2 consist of an average intensity taken 4.5 to $5.0 \mathrm{~ms}$ within the pulse cycle, whereas the afterpeak intensity values consist of an average taken 5.3 to $5.6 \mathrm{~ms}$; ca. $0.45 \mathrm{~ms}$ after 
power termination. When calculated, the ratio of the afterpeak-to-plateau intensity was found to vary as a function of the high-energy electronic state and the spatial position in the negative glow. This behavior can be explained by considering the various mechanisms leading to the population of the high-energy electronic states during the plateau and afterpeak time regimes. Generalizations regarding a series of electronic states are inferred from the 6 electronic regions depicted in the energy level diagram illustrated in Figure 3.3.3.1. The subsequent emissions originating from these six electronic states, Figure 3.3.3.2 and 3.3.3.3, represent emission behavior over different spatial regions in the negative glow. The mechanisms of the populating and depopulating processes of these various levels appeared previously in Bogaerts and Gijbels steady state model. ${ }^{26}$ 


\begin{tabular}{|c|c|c|c|c|c|}
\hline \multirow{2}{*}{ nm } & \multicolumn{3}{|c|}{ Intensity (mV) } & \multicolumn{2}{c|}{ Levels $_{(\mathbf{m})}$} \\
& Plateau & Afterpeak & Ratio & $\mathbf{E}_{\mathrm{L}}$ & $\mathbf{E}_{\mathrm{H}}$ \\
\hline \hline 223.85 & 1.92 & 12.22 & 6.36 & 1.64 & 7.18 \\
\hline 249.22 & 7.48 & 7.13 & 0.95 & 0.00 & 4.97 \\
\hline 261.84 & 9.26 & 25.67 & 2.77 & 1.39 & 6.12 \\
\hline 282.44 & 37.31 & 61.33 & 1.64 & 1.39 & 5.78 \\
\hline 307.38 & 4.44 & 5.89 & 1.33 & 1.39 & 5.42 \\
\hline 309.40 & 11.20 & 17.21 & 1.54 & 1.39 & 5.39 \\
\hline 319.41 & 9.62 & 11.57 & 1.20 & 1.64 & 5.52 \\
\hline 324.75 & 419.32 & 521.14 & 1.24 & 0.00 & 3.82 \\
\hline 327.40 & 269.29 & 341.30 & 1.27 & 0.00 & 3.79 \\
\hline 327.98 & 27.30 & 38.09 & 1.40 & 1.64 & 5.42 \\
\hline 329.28 & 35.88 & 45.85 & 1.28 & 1.39 & 5.15 \\
\hline 333.79 & 40.35 & 60.99 & 1.51 & 1.39 & 5.10 \\
\hline 338.54 & 2.36 & 13.67 & 5.78 & 3.79 & 7.45 \\
\hline 341.40 & 2.95 & 27.33 & 9.27 & 3.82 & 7.45 \\
\hline 348.16 & 4.84 & 39.97 & 8.25 & 3.79 & 7.35 \\
\hline 351.18 & 11.33 & 83.73 & 7.39 & 3.82 & 7.35 \\
\hline 353.04 & 23.75 & 40.02 & 1.69 & 1.64 & 5.15 \\
\hline 365.42 & 14.09 & 110.75 & 7.86 & 3.79 & 7.18 \\
\hline 368.74 & 18.13 & 184.69 & 10.19 & 3.82 & 7.18 \\
\hline 382.50 & 0.03 & 5.50 & 20.32 & 3.79 & 7.03 \\
\hline 386.18 & 2.24 & 10.81 & 4.84 & 3.82 & 7.03 \\
\hline 406.26 & 27.43 & 269.84 & 9.84 & 3.82 & 6.87 \\
\hline 406.32 & 57.04 & 366.80 & 6.43 & 3.82 & 6.87 \\
\hline 448.04 & 4.76 & 18.12 & 3.81 & 3.79 & 6.55 \\
\hline 510.55 & 84.60 & 112.64 & 1.33 & 1.39 & 3.82 \\
\hline 515.32 & 109.17 & 325.09 & 2.98 & 3.79 & 6.19 \\
\hline 521.82 & 170.75 & 500.75 & 2.93 & 3.82 & 6.19 \\
\hline 570.02 & 7.24 & 7.12 & 0.98 & 1.64 & 3.82 \\
\hline 578.21 & 11.87 & 13.85 & 1.17 & 1.64 & 3.79 \\
\hline 793.31 & 6.17 & 9.76 & 1.58 & 3.79 & 5.35 \\
\hline 809.26 & 11.02 & 18.65 & 1.69 & 3.82 & 5.35 \\
\hline & & & & & \\
\hline
\end{tabular}

Table 3-2 Copper atom plateau and afterpeak emission intensities taken at $6.0 \mathrm{~mm}$ from the cathode surface with the corresponding calculated afterpeak-to-plateau ratio and the transitions high $\left(E_{H}\right)$ and low $\left(E_{L}\right)$ energy levels 


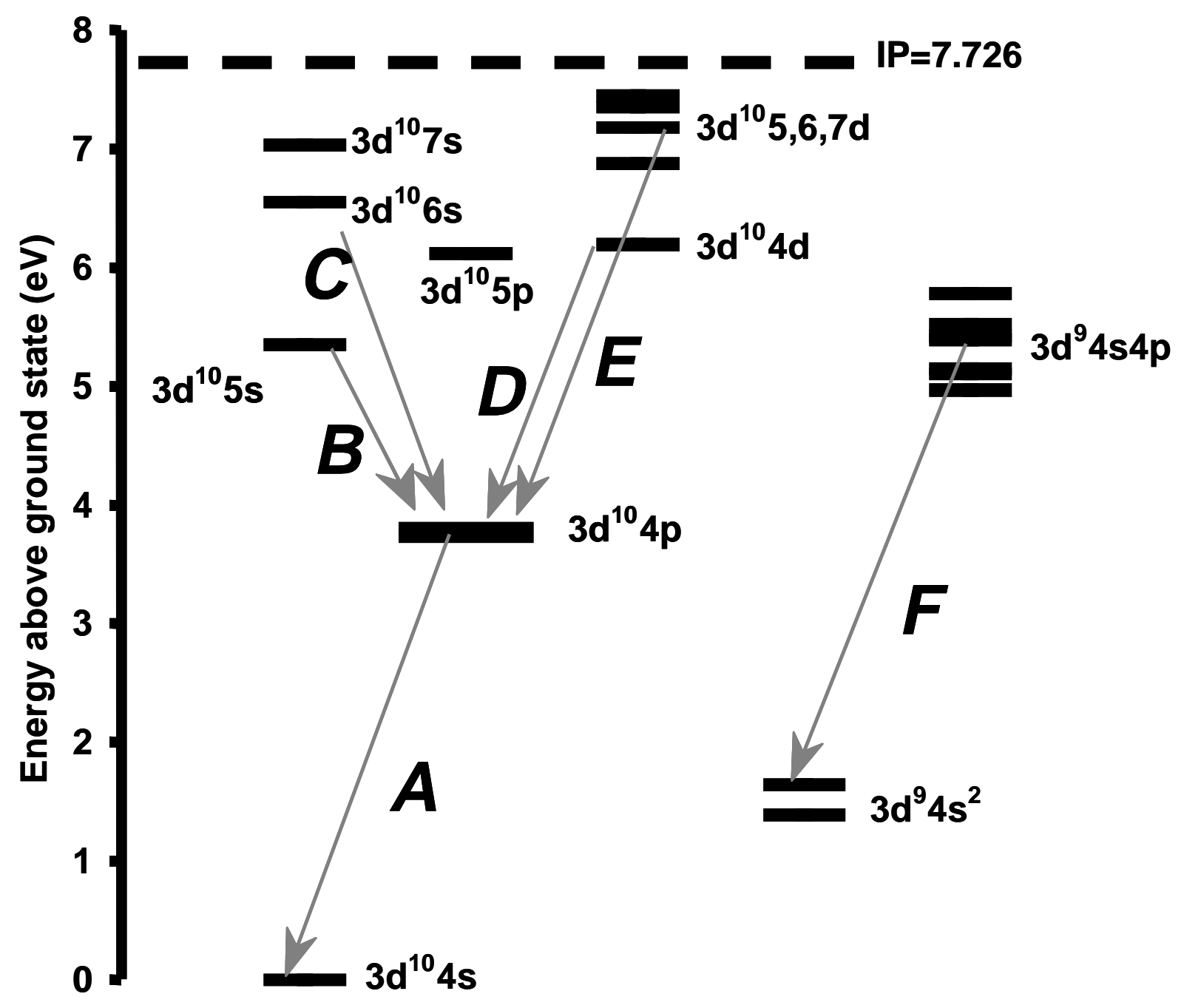

Figure 3.3.3.1 Energy Level diagram highlighting copper atom transitions of interest, labeled A through $\mathrm{F}$. 

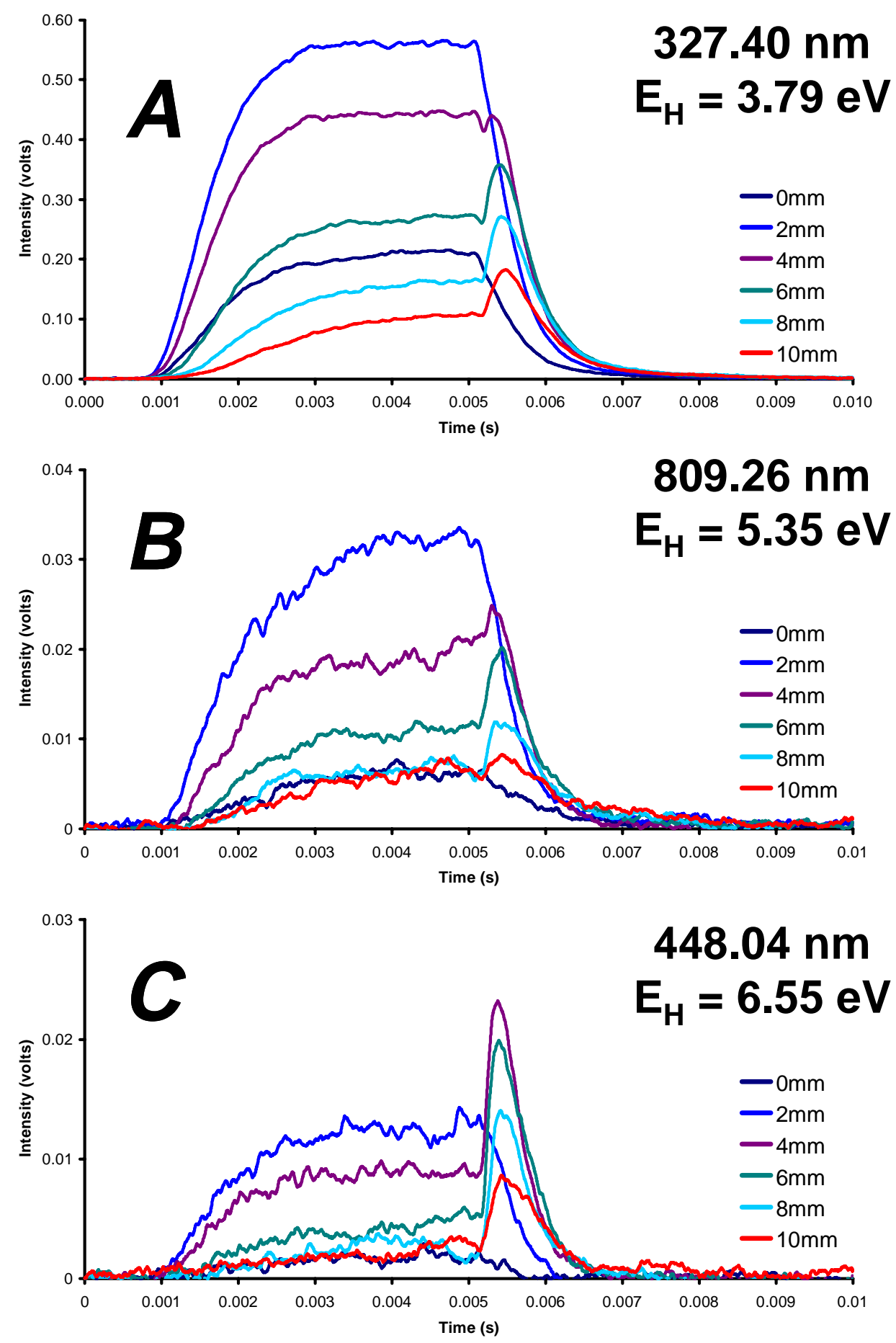

Figure 3.3.3.2 The temporal emission profiles of copper atom transitions as depicted in Figure 3.3.3.1, labeled A-C. Each transition demonstrates the change in the temporal emission profile moving vertically from the cathode surface in increments of $2 \mathrm{~mm}$. 

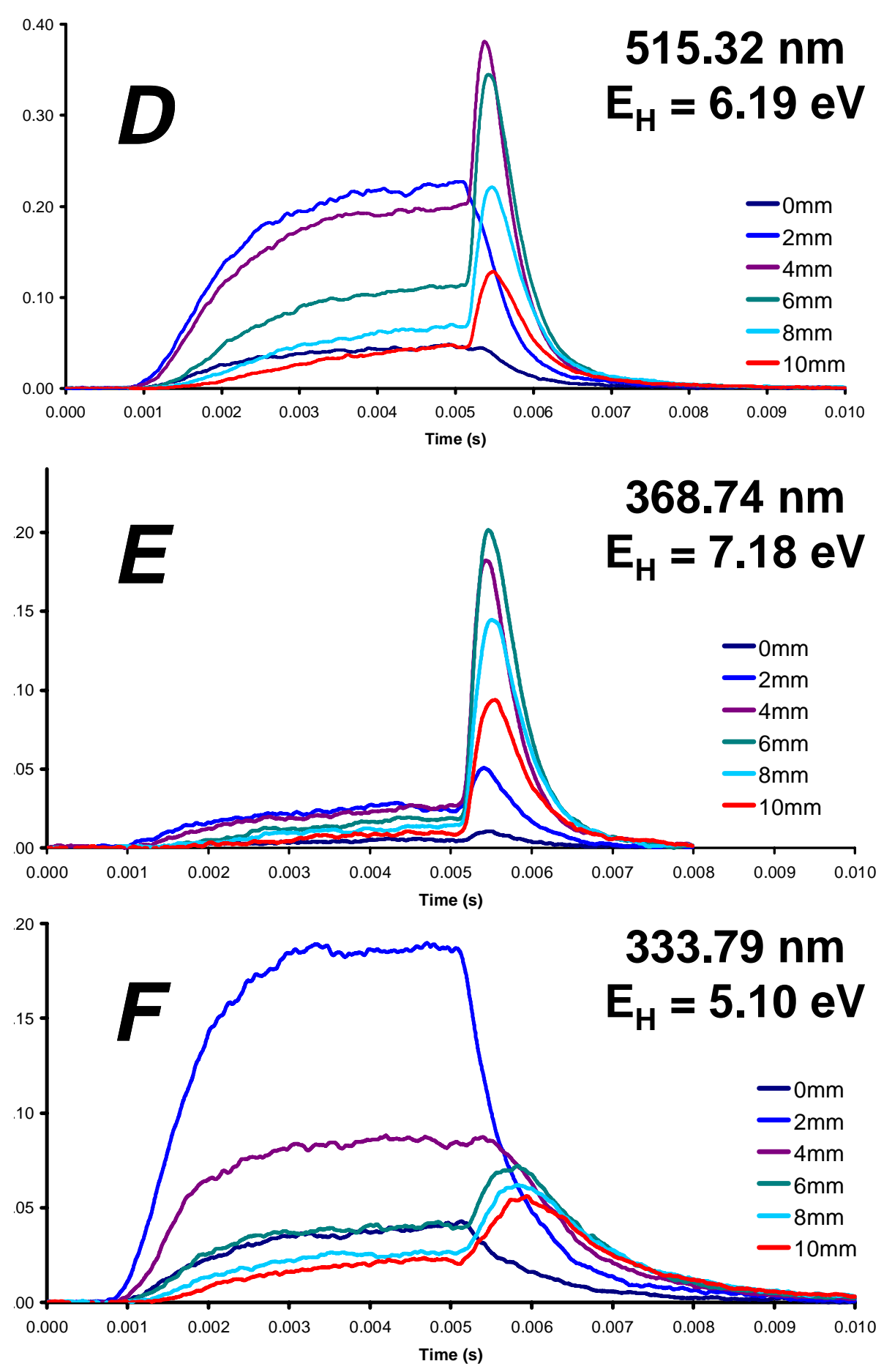

Figure 3.3.3.3 The temporal emission profiles of copper atom transitions as depicted in Figure 3.3.3.1 labeled D-F. Each transition demonstrates the change in the temporal emission profile moving vertically from the cathode surface in increments of $2 \mathrm{~mm}$. 


\subsubsection{Cu $3 d^{10} 4 p-4 s$}

Emission at $327.40 \mathrm{~nm}$ (Fig. 3.3.3.1) corresponds with the decay of the $3 d^{10} 4 p$ level to the atom ground state. This state occurs $3.79 \mathrm{eV}$ above the atom ground state with a transition probability of $1.37 \times 10^{-8} \mathrm{~s}^{-1} .{ }^{27}$ The large plateau intensity value evident in the temporal profiles of Figure 3.3.3.2 indicates that population of the $3 d^{10} 4 p$ level occurs predominantly through electron excitation. As previously mentioned, this plateau regime mimics steady state discharges. Emission throughout this temporal regime originates from species subject to electron excitation. The plateau intensity maximizes at a sampling distance of 2 $\mathrm{mm}$ from the cathode surface and decreases at greater distances. In contrast, the afterpeak emission maximum appears approximately $0.3 \mathrm{~ms}$ after power termination, and is not distinct until $4 \mathrm{~mm}$ from the cathode surface. Over the next $6 \mathrm{~mm}$, the afterpeak intensity decreases steadily but at a lower rate than the plateau intensity. Because the power was terminated and the lifetime is considered to be instantaneous, the most probable mechanism of populating this level is through recombination and radiative decay of higher energy states, most

notably through the $3 d^{10} 5,6$, and $7 d$ levels. The population of these levels will be discussed in the next section.

\subsubsection{2 $\mathrm{Cu}^{\circ}$ High Energy States}

The electronic states, labeled in Figure 3.3.3.2B through 3.3.3.3E, exhibit the effects of spatial and temporal position on emission intensity for electronic states of increasing energy. Each state decays into the $3 d^{10} 4 p$ level. The plateau intensity of each transition reaches an emission maximum within $2 \mathrm{~mm}$ of the 
cathode surface followed by a reduction in intensity at greater distances. This plateau spatial position coincides with the spatial maximum found in the $3 d^{10} 4 p-$ $4 s$ transition, with electron excitation of $\mathrm{Cu}^{\circ}$ being the primary mechanism. This electron excitation remains dominant until the plasma decays. During the afterpeak time regime, emission profiles increase to reach maxima at $5.3-5.5$ $\mathrm{ms}$, and $4-6 \mathrm{~mm}$ from the cathode surface. Upon power termination, thermalized electrons and copper ions recombine to produce highly excited copper atoms. These, once formed, radiatively decay to lower electronic states. This radiative relaxation cascade is the most probable mechanism by which the lower energy levels, such as the $3 d^{10} 4 p$, are populated once electron excitation halts in the afterpeak. This is consistent with previous reports that indicate the most probable production mechanism for copper ions in the afterpeak to be Penning ionization. ${ }^{12}$

\subsubsection{3 $\mathrm{Cu}^{0} 4 s 4 p-4 s^{2}$}

Emission originating from the radiative decay of $4 s 4 p$ states to the $4 s^{2}$ metastable copper atom states exhibits temporal afterpeak broadening as compared to other transitions studied. The reported transition probability of the $333.79 \mathrm{~nm}$ emission line, depicted in Figure 3.3.3.3F, is $3.80 \times 10^{5} \mathrm{~s}^{-1} .{ }^{27}$ This transition clearly has a much longer lifetime compared to the transitions previously discussed. During the plateau time regime, the major mechanism of excitation is electron excitation; whereas, the afterpeak intensity likely results from the radiative relaxation cascade from more highly excited copper atoms. The plateau and afterpeak intensity exhibit spatial maxima similar to that noted 
for the other electronic states discussed above. The temporal broadening is attributed to the longer natural lifetime of $0.0026 \mathrm{~ms}$, for this electronic state's decay to the copper atom metastable level.

\subsubsection{Afterpeak-to-plateau emission intensity ratio}

As alluded to in the above discussion, the ratio of copper atom afterpeakto-plateau emission intensity increases with increasing energy of the upper electronic energy level. Figure 3.3.3.4A is an illustration of this effect, using the ratio of afterpeak-to-plateau emission intensity (taken at $6 \mathrm{~mm}$ ) plotted as a function of the higher electronic energy level involved in a transition. This plot clearly shows that emission originating from higher electronic levels is heavily favored during the afterpeak time regime. Using the most intense transitions for various electronic levels observed, the ratio of afterpeak-to-plateau intensity is plotted as a function of distance, Figure 3.3.3.4B. At shorter distances, during both plateau and afterpeak time regimes, electron excitation dominates and the ratio is low. As distance increases, the ratio of afterpeak-to-plateau intensity increases with electronic energy levels. The most probable reason for this ratio increase is the production of copper ions by Penning ionization during the afterpeak time regime, followed by a loss in electron excitation. Plasma recombination processes in the afterpeak appear to maximize $6 \mathrm{~mm}$ from the cathode surface, this distance is needed for electrons to thermalize and is consistent with the spatial position of metastable argon atom population maxima observed in this laboratory. 
A closer inspection of Figures 3.3.3.4B and 3.3.3.4C, reveals a distinct jump in the calculated afterpeak-to-plateau ratio moving between electronic states of 5.78 and $6.12 \mathrm{eV}$. The explanation for this jump is that the electron excitation temperature during the plateau has a mean value greater than $5.78 \mathrm{eV}$ and less than $6.12 \mathrm{eV}$. This effect would limit the population of levels above 5.78 $\mathrm{eV}$ by electron excitation during the plateau time regime, leading to and increase in the calculated ratio. A previous report estimates electron temperatures in glow discharge plasmas to be on the order of $2-4 \mathrm{eV} .^{28}$ Future studies will examine the electron temperature inferred by this method for a variety of analyte materials. 


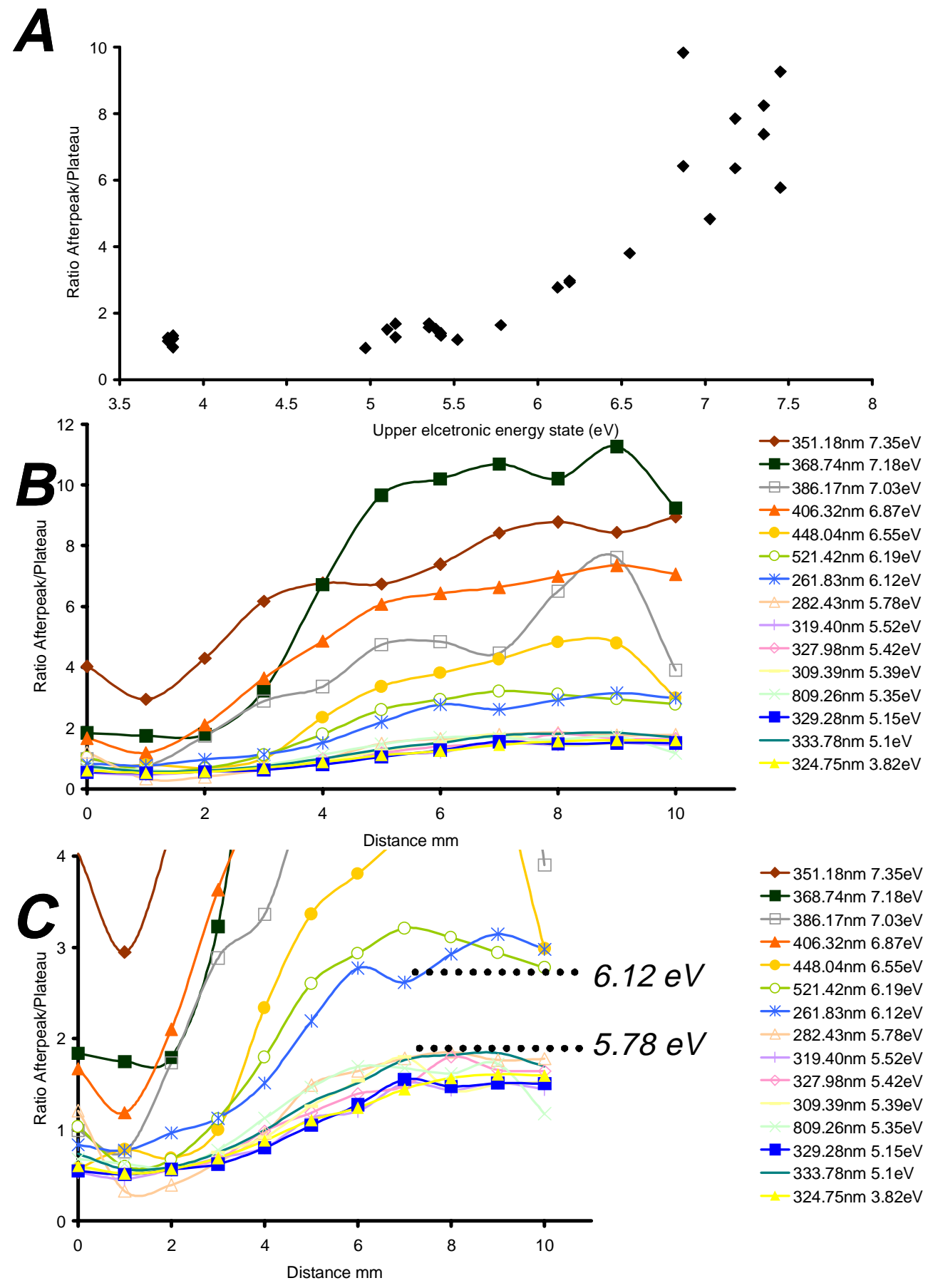

Figure 3.3.3.4 Calculated afterpeak to plateau ratio as a function of increasing; (A) transition energy above the ground state at $6 \mathrm{~mm}$, and (B) sampling distance above the cathode surface. Figure $C$ is an expanded view of Figure $B$ denoting a large jump in the calculated ratio. 


\subsubsection{Spatial Maps of Atomic Emission and Absorbance of Sputtered Analyte}

In order to clearly show the relative positions and temporal responses of the differing emission processes, spatial maps of atomic emission and absorbance were collected under the same glow discharge conditions over a series of times in the pulse cycle. Each map consists of 368 spatial positions extending $10 \mathrm{~mm}$ laterally and $18 \mathrm{~mm}$ horizontally from the cathode's surface. The low and highenergy emission maps were selected based on intensity values and constructed by monitoring the emission from the $324.75 \mathrm{~nm}, 3.82 \mathrm{eV}$, and $368.74 \mathrm{~nm}, 7.12$ $e V$ transition, corresponding to emissions from the $3 d^{10} 4 p$ and $3 d^{10} 6 d$ electronic states.

\subsection{Ground State Copper Atom Absorbance Maps}

The maps in Figure 3.3.3.5 illustrate the two-dimensional distribution of ground state copper atoms throughout the pulse cycle. The large rectangle of low absorbance extending $0.5 \mathrm{~mm}$ vertically and $\sim 4 \mathrm{~mm}$ horizontally from the origin is easily identifiable as the sample cathode and ceramic Macor shield. When power is applied, argon ions form through electron ionization and begin to sputter the cathode surface. At $1.5 \mathrm{~ms}$ into the pulse cycle the sputtering process has only just begun, over the next $3.0 \mathrm{~ms}$ the sputtered atom cloud extends outward, filling the glow discharge chamber. At this time a steady state population is reached that remains constant until $5.5 \mathrm{~ms}$ into the pulse cycle, $0.5 \mathrm{~ms}$ past power termination. During power application, the absorbance maximum is centered $1.5 \mathrm{~mm}$ above the edge of the cathode surface and radiates spherically outward. Each map exhibits a dip in absorbance intensity close to the cathode 
surface, which has been observed in previous absorption, fluorescence and modeling studies. ${ }^{29,30,31}$ As copper atoms sputter off the cathode surface they are subject to collisions with the argon bath gas. These collisions result in thermalization or a dampening of the initial kinetic energy that the sputtered atoms possessed upon leaving the cathode surface ${ }^{32}$ Once thermalized, the ground state copper atoms diffuse radially into the negative glow where they are subjected to collisional excitation and ionization processes. These maps demonstrate that a steady state population of copper atoms exists throughout the plateau and afterpeak time regime. Most notably, this shows that the spatial position of excitation and ionization of copper is not related to copper atom diffusion, but instead related to the diffusion of the collision partner, i.e. fast electron or metastable argon atom. 

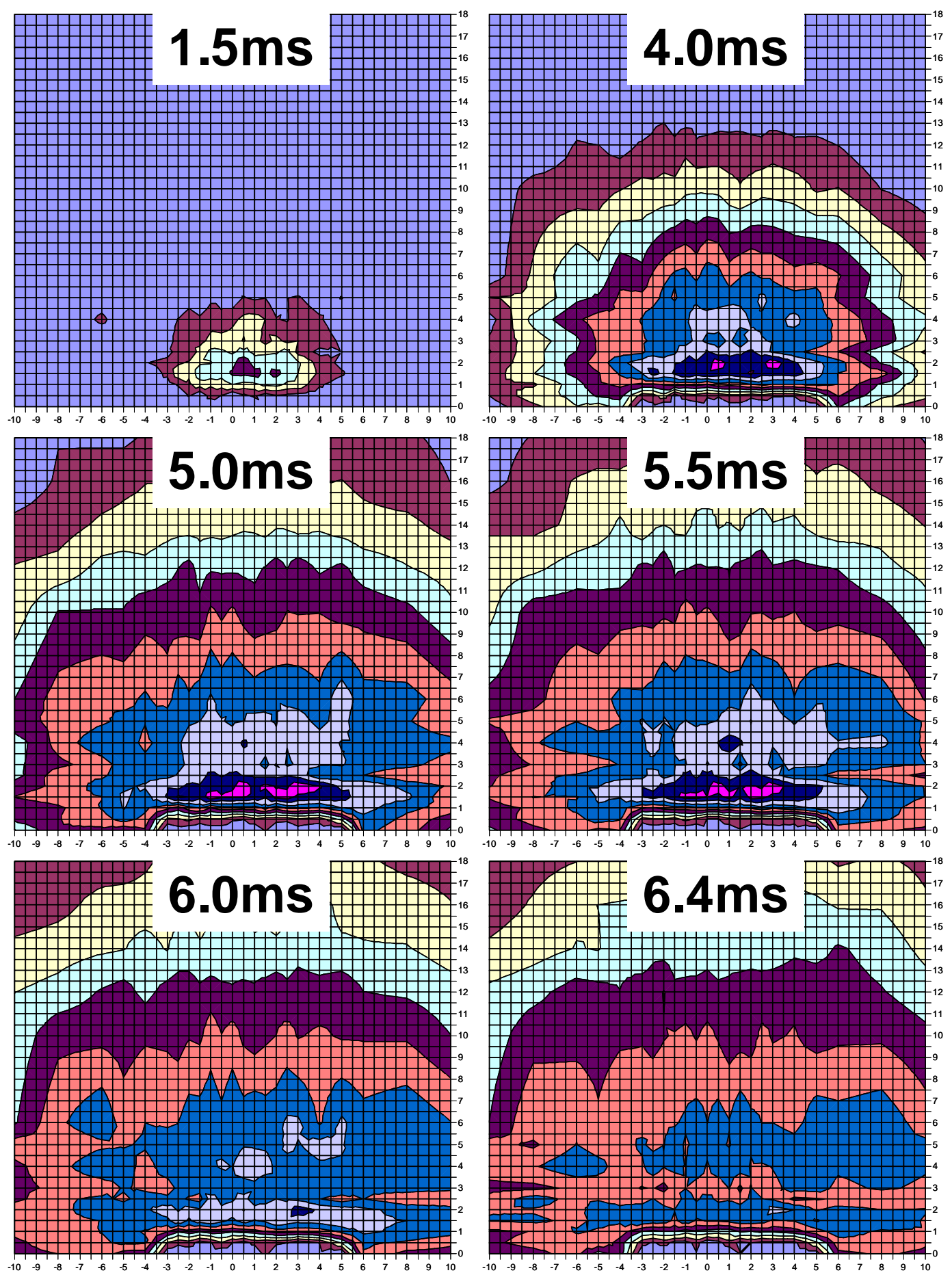

Figure 3.3.3.5 Two dimensional absorbance maps of the copper atom population monitored through out the pulse cycle using hollow cathode emission at $324.75 \mathrm{~nm}$. 


\subsection{Copper Atom Emission Maps}

The spatial and temporal responses of emission from the $3 d^{10} 4 p$ and $3 d^{10} 6 d$ electronic states are characterized in the maps shown in Figure 3.3.3.6 and 3.3.3.7. The low-lying electronic state of $3 d^{10} 4 p$ is $3.82 \mathrm{eV}$ above the copper atom ground state. In contrast, the $3 d^{10} 6 d$ electronic state is substantially higher at $7.18 \mathrm{eV}$. Each set of temporal maps is normalized to the highest intensity value during the pulse cycle for that particular transition, and relative changes to this maximum are reflected in multiples of $10 \%$ of its value. Both sets of maps show that the plateau intensity reaches a steady state value 3.0 to $4.0 \mathrm{~ms}$ into the pulse cycle. However, the relative intensity for each is quite different. At 3.0 ms into the pulse cycle the $3 d^{10} 4 p(3.82 \mathrm{eV})$ emission reaches 90 to $100 \%$ of its pulse cycle temporal maximum and extends radially outward. This emission sphere remains constant over the next $2.0 \mathrm{~ms}$ until $0.1 \mathrm{~ms}$ past power termination. Comparison with the $3 d^{10} 6 d(7.18 \mathrm{eV})$ emission, however, demonstrates a local maximum over the plateau regime that is only $20-30 \%$ of its temporal maximum. The relative positions of these two plateau maxima coincide with the edge of the ground state atom population observed in the previous absorption maps. As discussed in a previous section, electron excitation is the dominant excitation mechanism during the plateau time regime. Because of the relative magnitude of the energy states involved, electron excitation is the most probable mechanism for populating the $3 \mathrm{~d}^{10} 4 \mathrm{p}(3.82 \mathrm{eV})$ electronic state. Populating the $3 d^{10} 6 d(7.18 \mathrm{eV})$ electronic state at this time through electron excitation would require an electron of significantly higher energy or multiple 
excitation steps. Both temporal transition maps do not show significant changes in intensity until $5.1 \mathrm{~ms}$ into the pulse cycle. At this point, emission from the $3 d^{10} 4 p(3.82 \mathrm{eV})$ electronic state begins to decline from its temporal maximum. During this decline, the center of intensity shifts further away from the cathode into the negative glow. This change in position occurs over a $0.6 \mathrm{~ms}$ time period, with the center of the population maximum moving from $2.5 \mathrm{~mm}$ to $5.5 \mathrm{~mm}$ from the cathode surface. This shift in spatial position occurs with a reduction in intensity to $30-40 \%$ from the temporal maximum. Emission from the $3 d^{10} 6 d(7.18$ $\mathrm{eV}$ ) electronic state, however, demonstrates opposite behavior. Between 5.1 and $5.5 \mathrm{~ms}$, emission originating from this electronic state increases in both area and intensity reaching $90-100 \%$ of its temporal maximum. This temporal maximum is located $6 \mathrm{~mm}$ above the cathode surface and radiates outward in all directions. Absorbance measurements show no significant movement of the copper atom ground state population during this time. The relative shifts of these electronic transitions and changes in intensity must be attributed to changes in excitation processes.

The shift in the emission from $3 d^{10} 4 p(3.82 \mathrm{eV})$ electronic state can be explained by the radiative decay of higher electronic states, such as the $3 d^{10} 6 d$ $(7.18 \mathrm{eV})$ state, cascading to populate this level. Excitation to the $3 \mathrm{~d}^{10} 6 \mathrm{~d}(7.18$ $\mathrm{eV}$ ) state during the afterpeak time regime arises from recombination of copper ions with thermal electrons producing highly excited copper atoms. The spatial and temporal position at which this population maximizes matches metastable argon maps that have been previously produced. Metastable argon atoms are 
produced through a similar process where argon ions recombined with thermal electrons. Once formed, metastable argon atoms may lead to an increase the in copper ion ground state through Penning ionization. The afterpeak intensity of the $3 d^{10} 6 d(7.18 \mathrm{eV})$ continues to $5.6 \mathrm{~ms}$ and begins to collapse over the next 1.0 ms. The position of the collapse is localized ca. $6 \mathrm{~mm}$ and does not demonstrate any shift caused by atom diffusion. 

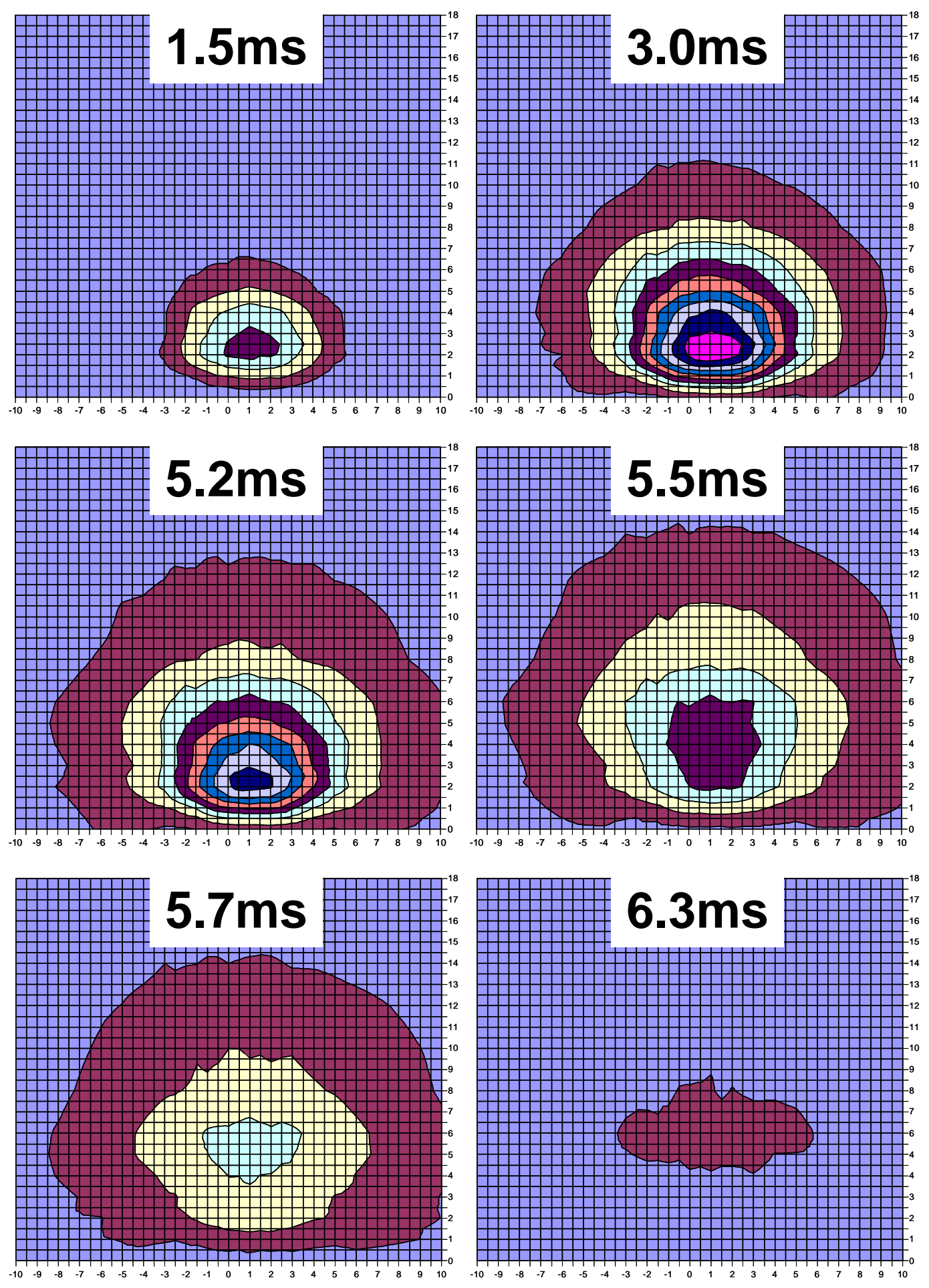

Figure 3.3.3.6 Two dimensional maps of a low-lying atom electronic state $3.82 \mathrm{eV}$ above ground state, monitored by emission at $324.75 \mathrm{~nm}$, through out the pulse cycle. 

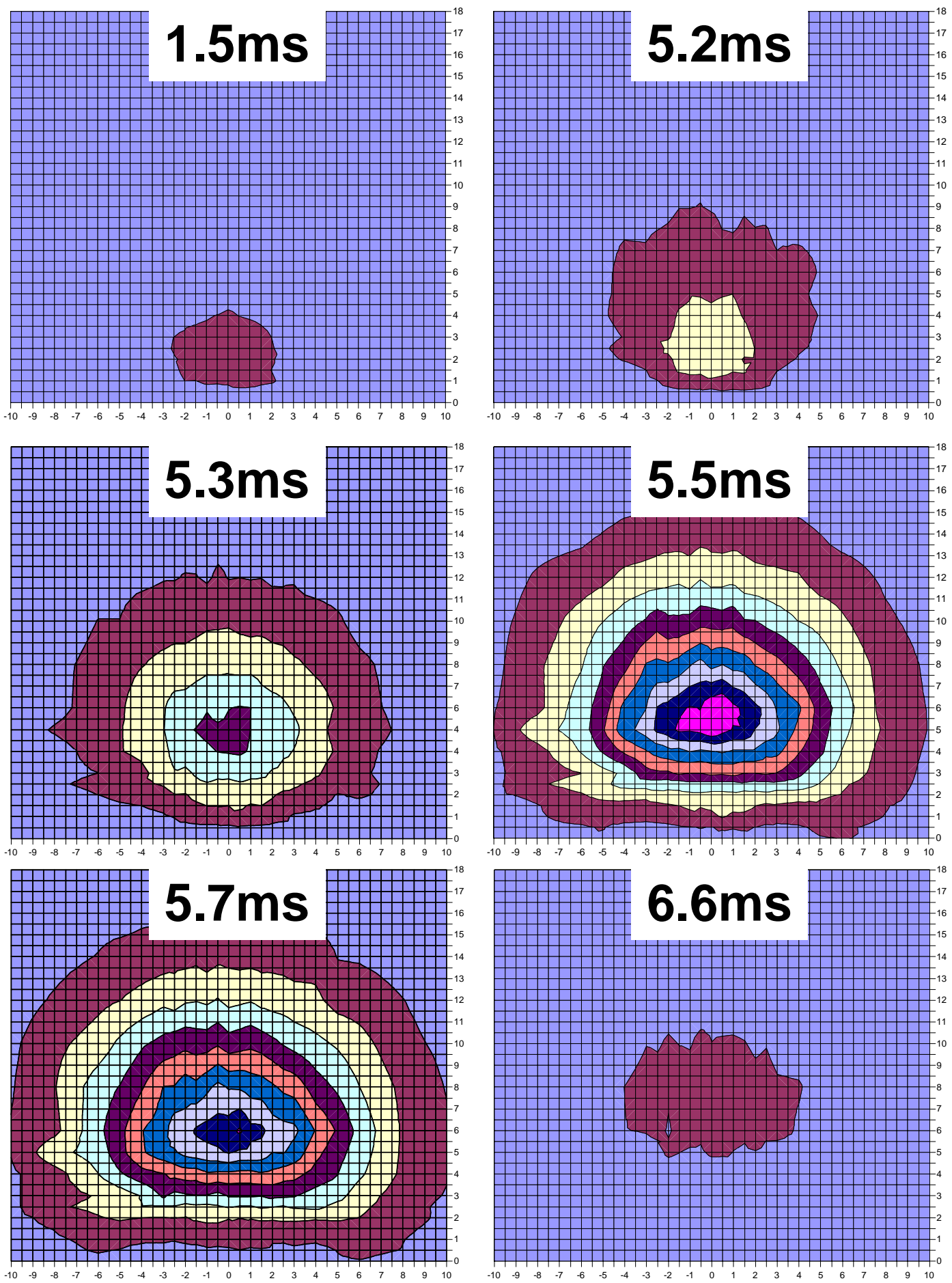

Figure 3.3.3.7 Two-dimensional maps of a high lying atom electronic state $7.18 \mathrm{eV}$ above the ground state, monitored by emission at $368.7 \mathrm{~nm}$,throughout the pulse cycle. 


\subsubsection{Classification of Copper Ion Electronic States}

An investigation of the copper ion lines was carried out in a similar manner as the copper atom transitions. The highest energy state that was observed occurred $10.99 \mathrm{eV}$ above the ion ground state. All transitions exhibit emissions only during the plateau time regime, that is none demonstrate the afterpeak characteristic observed in atom transitions. These results conflict with previous reports from this laboratory, by which cooper ion emissions were mistakenly assigned. Of the observed transitions the $224.70 \mathrm{~nm}$ line shows unique behavior when compared with all other electronic transitions collected and will be discussed separately. Generalizations regarding the remaining electronic states are inferred from the 2 electronic regions depicted in the energy level diagram illustrated in Figure 3.3.4.1. The subsequent emissions originating from these two electronic states are depicted in Figure 3.3.4.2 and represent emission behavior over different spatial regions in the negative glow. Each transition reaches the steady state, plateau, intensity within 2.0 to $3.0 \mathrm{~ms}$ into the pulse cycle. The relative position for each maximum depends once again on the highenergy electronic level of the transition. 


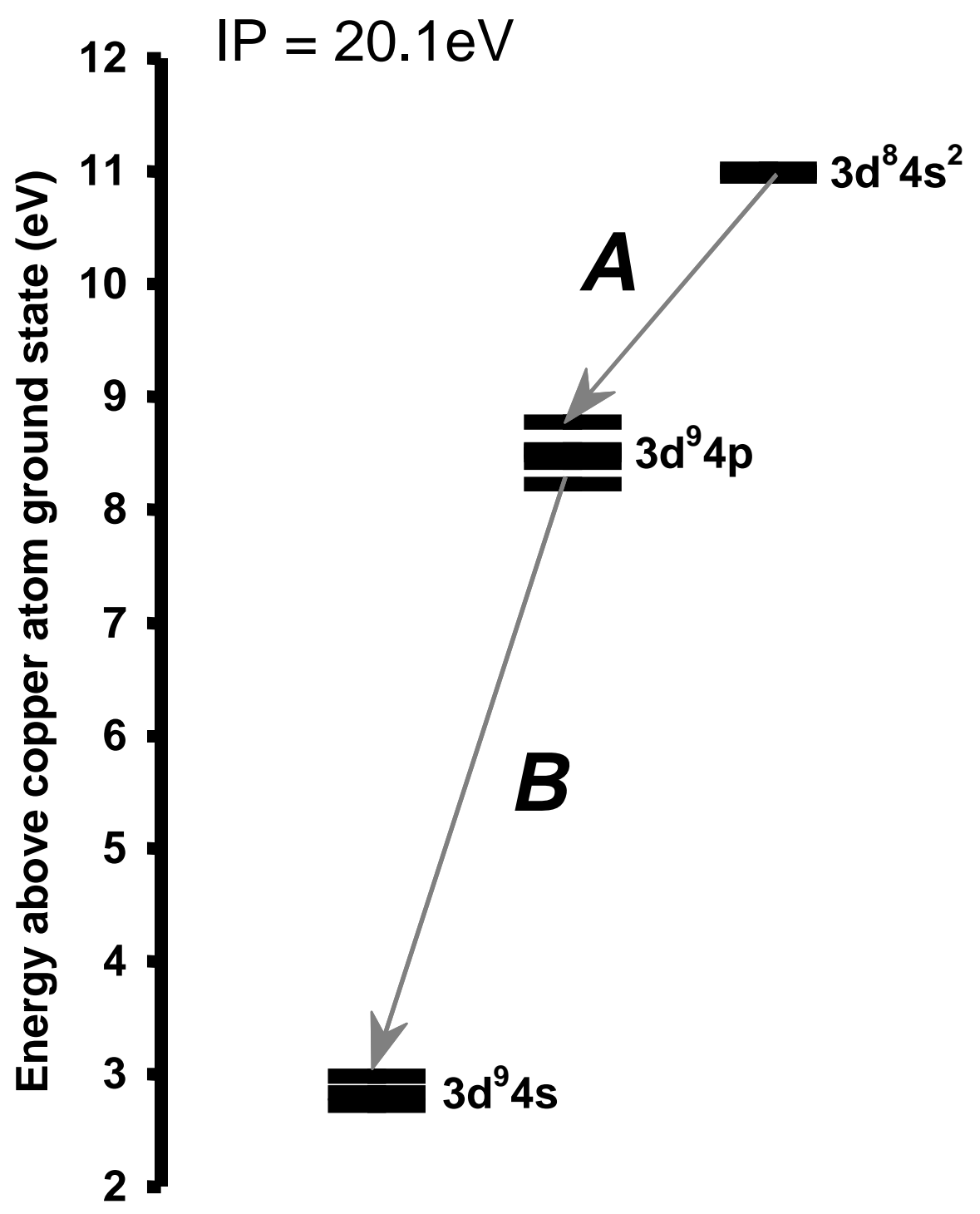

Figure 3.3.4.1 Energy level diagram highlighting copper ion transitions of interest labeled A though B (see Figure 3.3.4.2) 

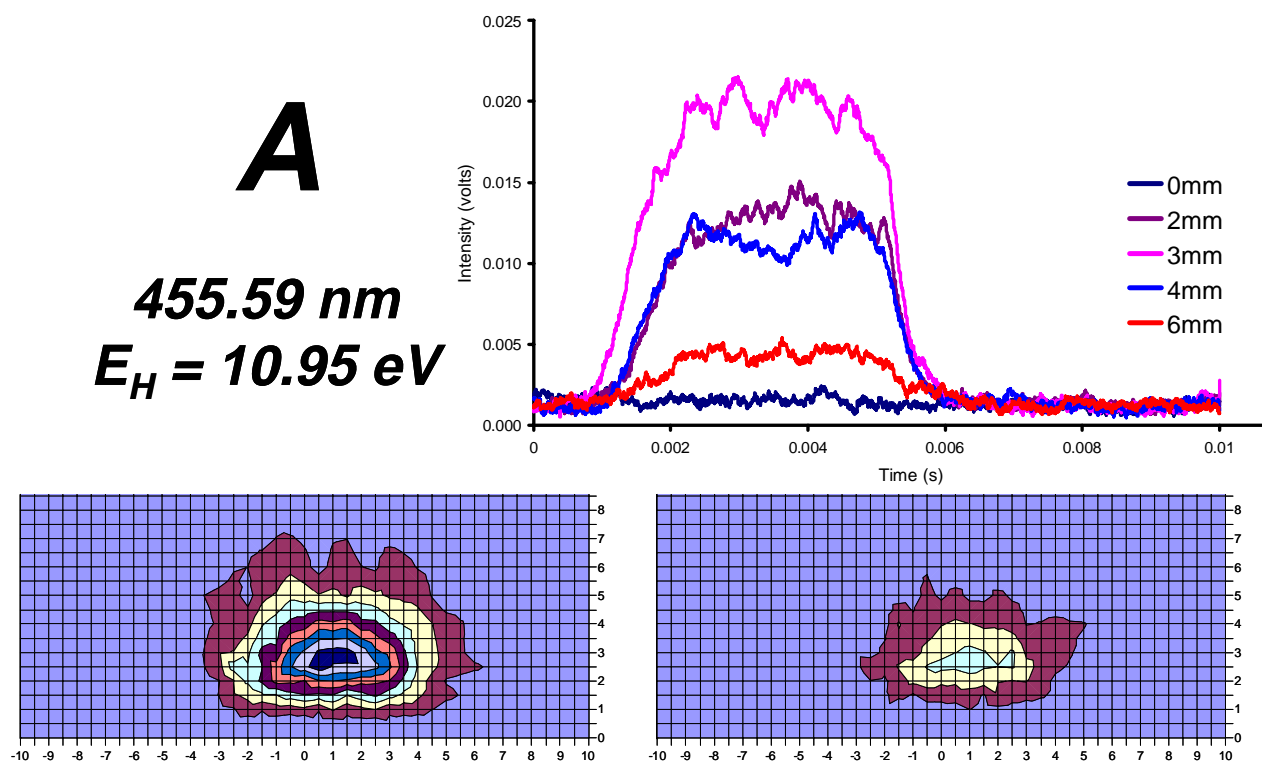

$5.0 \mathrm{~ms}$

$5.4 \mathrm{~ms}$

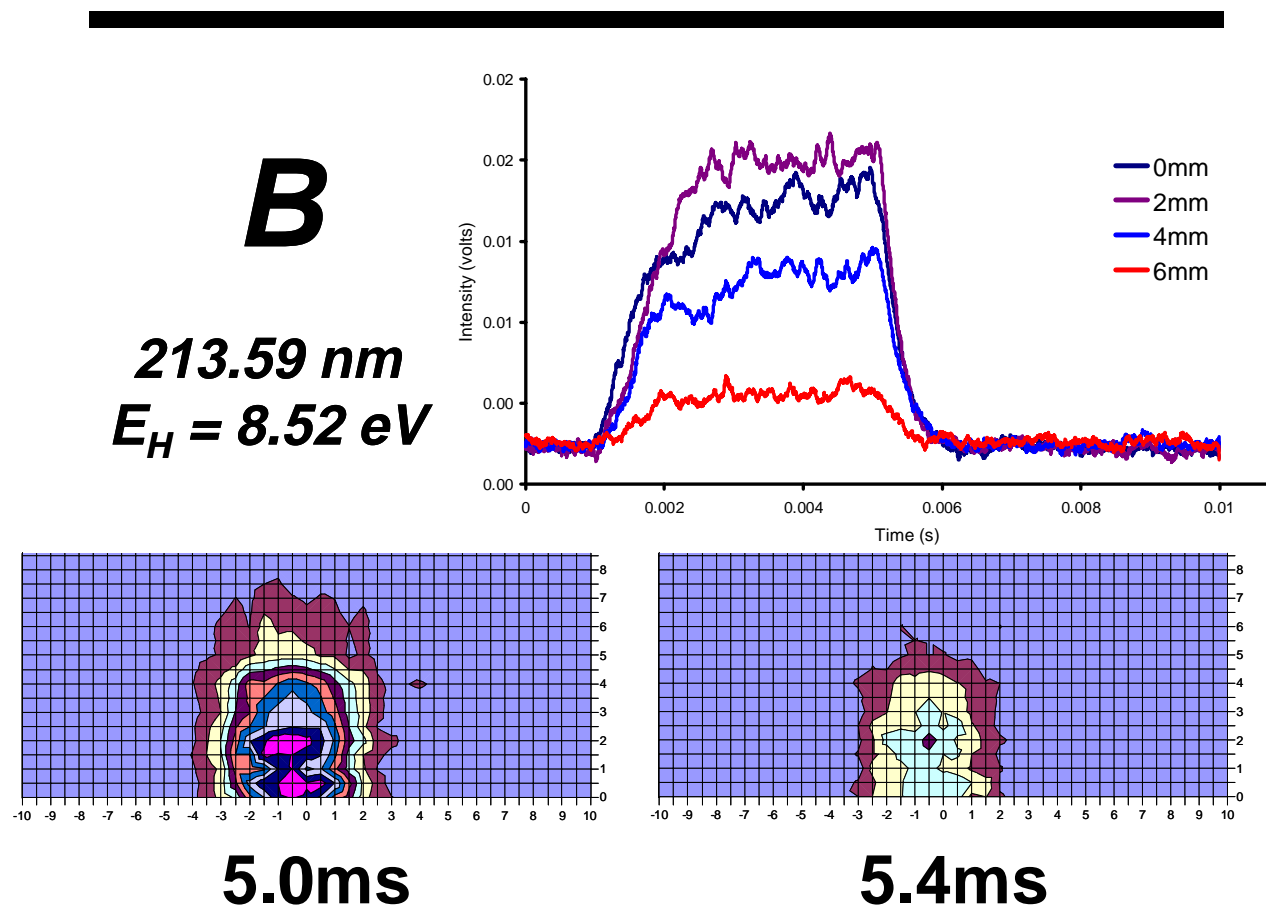

Figure 3.3.4.2 Temporal profiles of copper ion transition as depicted in Figure 3.3.4.1, labeled A and B. Each transition demonstrates the change in temporal emission profile moving vertically from the cathode surface in increments of $2 \mathrm{~mm}$. Below each are 2-dimensaonal emission maps taken at $5.0 \mathrm{~ms}$ and $5.4 \mathrm{~ms}$ 
The high-energy state, $3 d^{8} 4 s^{2}$, occurs $10.95 \mathrm{eV}$ above the ion ground state and is monitored by emission at $455.59 \mathrm{~nm}$, Figure 3.3.4.2A. The behavior of this line is indicative of the other $3 d^{8} 4 \mathrm{~s}$ transitions. The adjacent map, $5.0 \mathrm{~ms}$ into the pulse cycle, demonstrates a discrete maximum $3 \mathrm{~mm}$ from the cathode surface. This spatial distance overlaps well with argon ion emission measurements made in this laboratory. The processes most likely to populate these levels involve ionization followed by electron excitation. This two-step process explains the absence of any ion emission in the afterpeak because electron excitation processes have stopped. Previous reports on metastable argon atom populations during the plateau and steady state plasmas indicate a large population of metastables within $1-2 \mathrm{~mm}$ of the cathodes surface. In this report, electron excitation of copper atoms has been deduced to occur at slightly larger distances. It is uncertain, however, to what degree Penning ionization is involved in the ionization of copper atoms in this two-step mechanism. The adjacent temporal map at $5.4 \mathrm{~ms}$ demonstrates no appreciable change in spatial position during the plateau and afterpeak time regimes. The $3 d^{9} 4 p$ levels are characterized, Figure 3.3.4.2B, by the $213.60 \mathrm{~nm}$ transition that occurs $8.52 \mathrm{eV}$ above the ion ground state. The most probable mechanism for populating this level is ionization followed by electron excitation followed by radiative decay from the $3 d^{8} 4$ s levels. Each transition from the $3 d^{9} 4 p$ levels exhibits a broad spatial maximum occurring 1 - $2 \mathrm{~mm}$ from the cathode surface. This indicates that there is no significant diffusion during the afterpeak time regime. 
Emission from the $224.70 \mathrm{~nm}$ line, originating from the $3 \mathrm{~d}^{9} 4 \mathrm{p}$ level $(8.23$ $\mathrm{eV})$, exhibits a different behavior from that of any other copper ion electronic state studied, Figure 3.3.4.3. The mechanism and characterization of this transition has been reported previously as charge transfer between a metastable argon ion, $3 p^{52} \mathrm{P}_{3 / 2}(15.76 \mathrm{eV})$, and ground state copper atom. ${ }^{33,34}$ This process is very selective because the energy deficit between these two species is only $\Delta E$ $=-.02 \mathrm{eV}$. It is important to note that emission at $229.40 \mathrm{~nm}, 3 \mathrm{~d}^{9} 4 \mathrm{p}(8.23 \mathrm{eV})$, was not observed, since it to would be also be resonant with charge transfer. The spatial maximum of the $224.70 \mathrm{~nm}$ emission occurs $5 \mathrm{~mm}$ from the cathode surface at $5.0 \mathrm{~ms}$ into the pulse cycle. Unlike any atom or ion temporal profiles collected previously, the emission intensity during the $5.0 \mathrm{~ms}$ power pulse never reaches a steady state value. Because copper atom absorbance maps have demonstrated a steady state ground state population in the plasma within $4.0 \mathrm{~ms}$ of pulse initiation, the absence of steady state behavior here is attributed to the production of the metastable argon ion, $3 p^{52} \mathrm{P}_{3 / 2}(15.76 \mathrm{eV})$. No afterpeak behavior was observed for this copper ion transition, as was the case for other copper ion transitions studied. A further investigation of this transition is planned. 

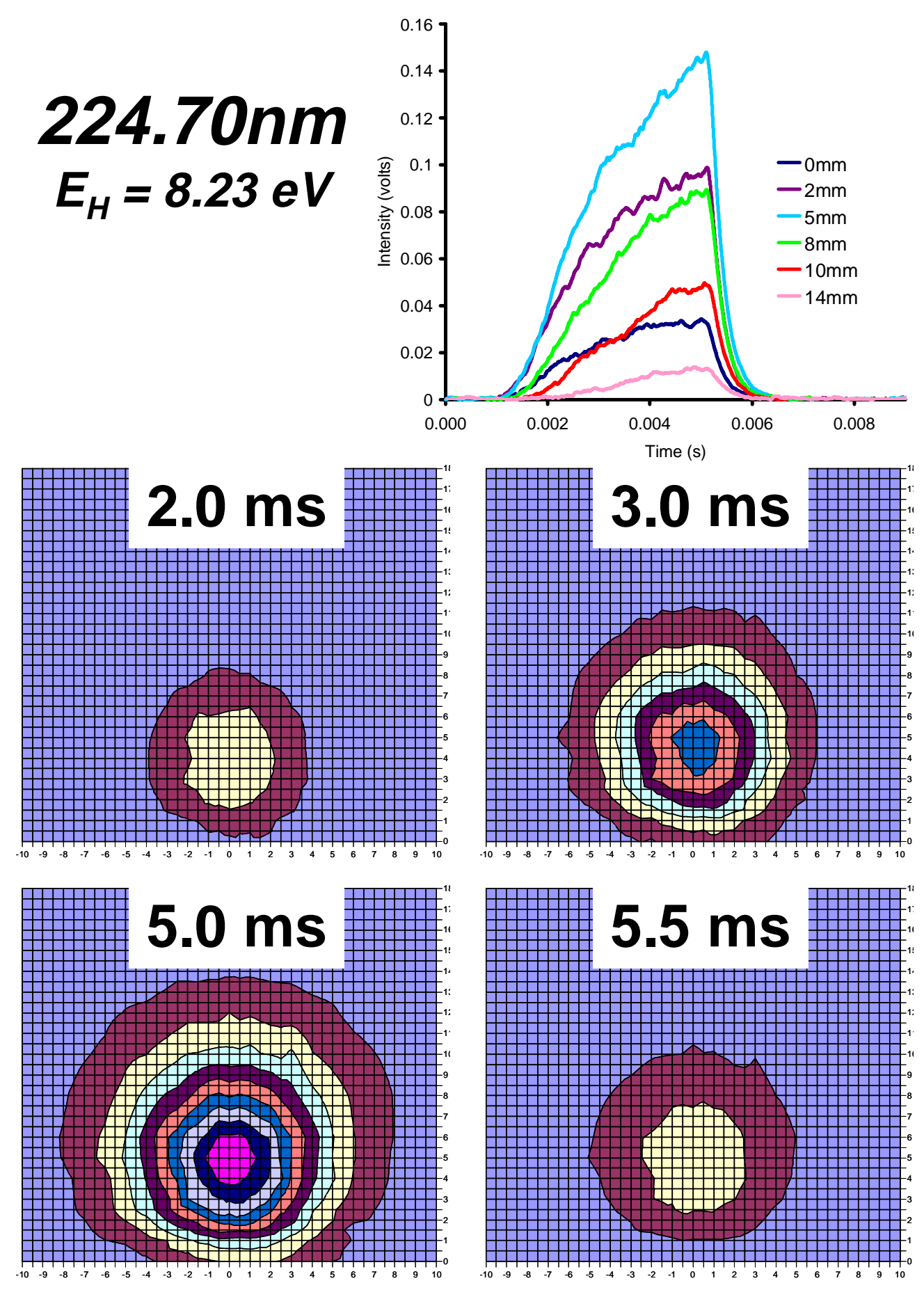

Figure 3.3.4.3 Temporal emission traces at increasing cathode distances of copper ion emission monitored at 224.7nm. Emission maps were constructed throughout the pulse cycle. 


\subsubsection{Effects of Pressure and Power}

Changes in both pressure and power affect excitation and ionization as well as sputtering processes. These two variables control the mean free path of both electrons and discharge species, as well as the energetics of argon ions bombarding the cathode surface. Changing either will affect the relative spatial and temporal position of excitation and ionization processes. A series of optical maps were constructed in order to investigate both power and pressure for discharge gas and sputtered species through out the pulse cycle. These studies were done independent of one another, and are meant to show the relative changes. Each pressure map was collected under a constant power of 1.5 watts under pressures of $0.5,0.8$ and 1.0 torr argon working gas. Power maps were collected under a constant pressure of 0.8 torr, with increasing pulse powers of $0.5,1.3$ and 2.3 watts.

\subsubsection{Argon lon}

Temporal argon ion emission maps were constructed by monitoring emission at $476.4 \mathrm{~nm}$, corresponding to an electronic transition $19.76 \mathrm{eV} \rightarrow 17.26$ $\mathrm{eV}$ above the argon ion ground state. Figure 3.3.5.2 demonstrates the confinement of emitting species with increasing pressure during the plateau, 5.0 $\mathrm{ms}$, and afterpeak, $5.5 \mathrm{~ms}$. As discussed earlier, the mechanism for populating this transition state is electron excitation of an argon ion species by high-energy electrons. This confinement is due to the decrease of the mean free path with increasing working gas pressure, as high-energy electrons are thermalized closer to the cathode surface. The spatial maps taken during the afterpeak at $5.5 \mathrm{~ms}$ 
show a reduction in emission intensity and no appreciable diffusion. The relative changes in emission intensity illustrate a much more rapid thermalization process at higher pressures.

The two-dimensional maps taken under constant pressure, Figure 3.3.5.2, demonstrate the effects of power on this transition. Working in the abnormal range of glow discharge, increasing power is accompanied by an increase in both current and voltage. This results in a larger population of argon ions and electrons traveling at higher velocities. These two effects result in the larger emitting radiance found in each map during the plateau time regime, $5.0 \mathrm{~ms}$. The position of the spatial intensity maximum appears unchanged at this time, and is centered ca. 2 - $3 \mathrm{~mm}$ from the cathode surface. Maps taken during the afterpeak time regime, $5.5 \mathrm{~ms}$, demonstrate no applicable diffusional effects as the emission intensity decays. 

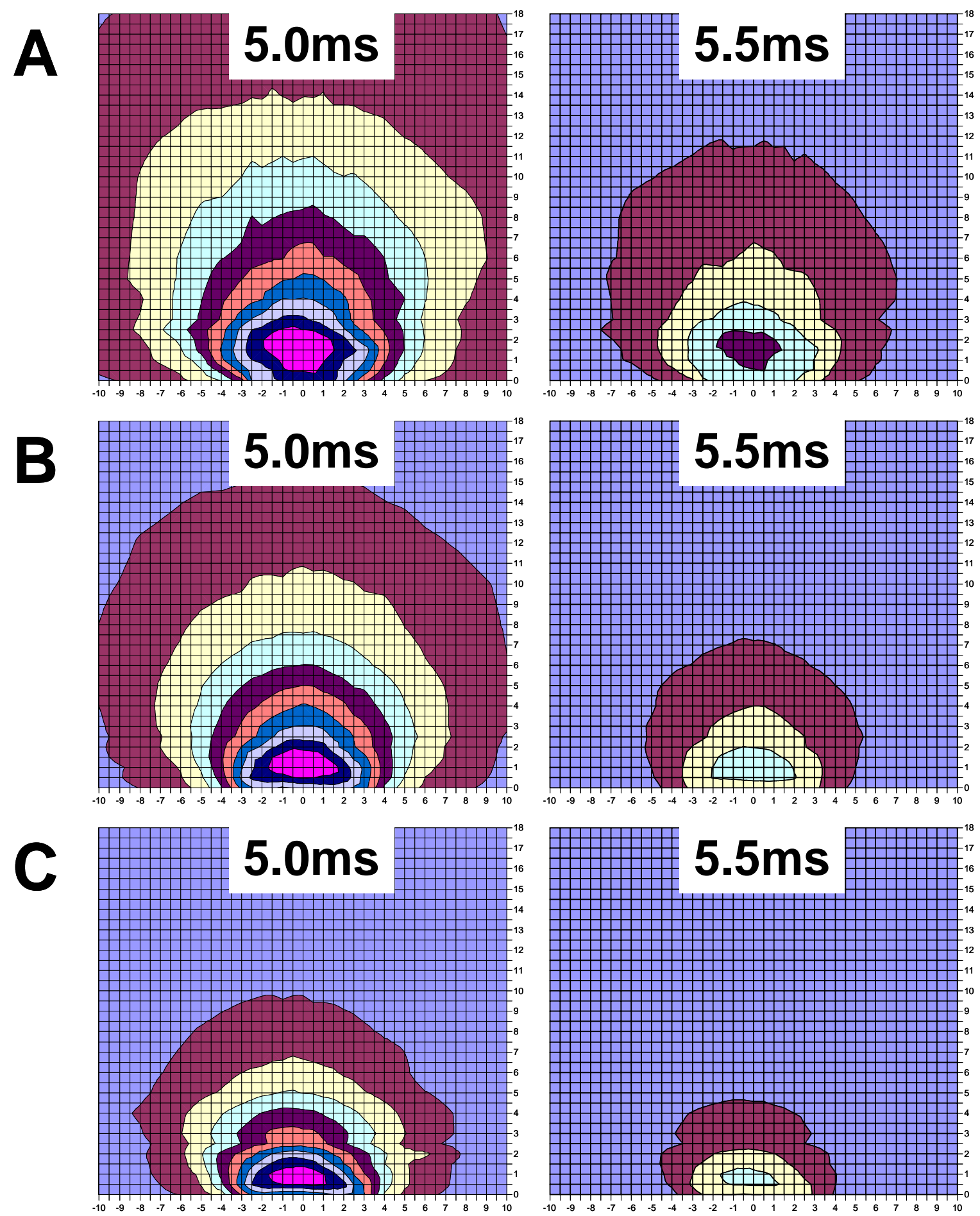

Figure 3.3.5.1 Two dimensional emission maps of argon ion monitored by emission at $476.4 \mathrm{~nm}$ at a constant power of 1.5 watts and pressures of $(A) 0.5$, (B) 0.8 , and (C) 1.0 torr. 

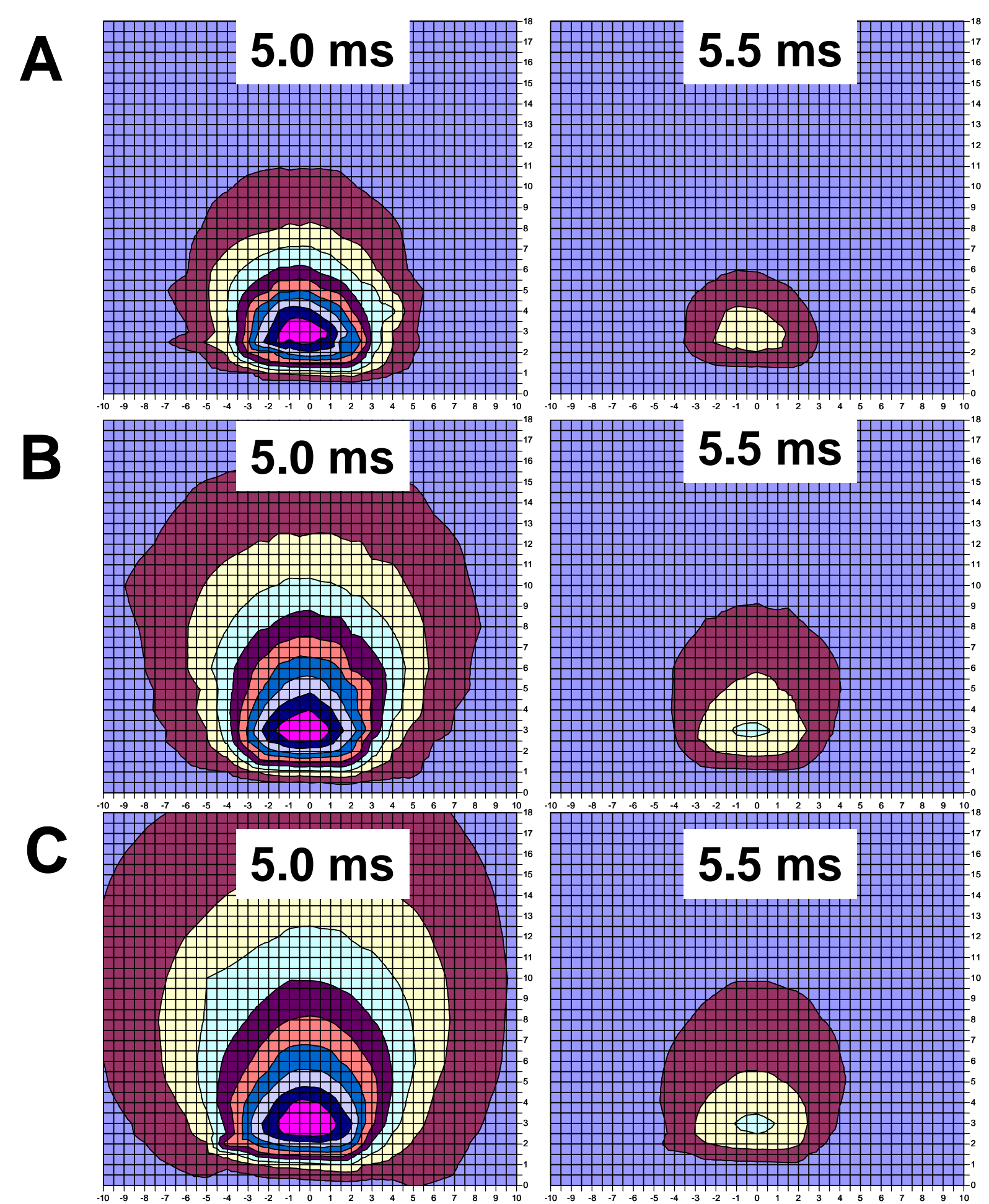

Figure 3.3.5.2 Two dimensional emission maps of argon ion monitored by emission at $476.4 \mathrm{~nm}$ at a constant pressure of 0.8 torr and power of $(A) 0.5,(B)$ 1.3 , and (C) 2.3 watts. 


\subsubsection{Metastable Argon Atom}

Power and pressure effects on the argon metastable population were studied by monitoring plasma emission at $811.5 \mathrm{~nm}$. The emission at $811.5 \mathrm{~nm}$ is the radiative decay of excited argon atoms to the metastable level. During all measurements, a large plateau value dominated emission and was located 0.5 to $1.5 \mathrm{~mm}$ from the cathode surface. For this reason two series of maps are presented, one in which the intensity is normalized throughout the pulse cycle and one which in which the spatial intensity is normalized at that particular time.

Pressure effects are presented in Figures 3.3.5.3 and 3.3.5.4 that show the normalized and raw pulse cycle maps. In order to maintain a constant power, decreasing pressure results in an increase in the applied voltage, and decrease in current. This results in a fewer number of charge carriers with an increased kinetic energy. The fewer charge carriers, argon ions, travel faster. This coupled with the longer mean free path suggests that recombination process at low pressures are a lower probability process. At very low pressures, emission intensity was found to dominate around the cathode surface, and no distinguishable afterpeak was observed. At this pressure the dominant mechanism for metastable argon atom production appears to be excitation and not recombination. The raw data map Figure 3.3.5.4, does show that emission from recombination processes occur at larger distances and at longer times. This small afterpeak is $\sim 1-5 \%$ of the emission intensity found during the plateau time regime. This large distance and longer time is due to the thermalization process of electrons, which have a higher kinetic energy and must travel further 
into the negative glow before losing sufficient energy to favor recombination. As pressure is increased, $0.8-1.0$ torr, a confinement the emission sphere is observed in both series of maps. This confinement is a result of electrons being thermalized closer to the cathode surface. The relative intensity changes between the three pressure maps illustrate that recombination process increase with pressure. The raw pulse cycle data exhibit a shift in the location of emission as processes change from excitation, in the plateau, to recombination processes, in the afterpeak.

As power is increased, emission during the both plateau and afterpeak time regimes increases in a linear fashion. The larger argon ion population present with increasing powers and currents can explain this emission increase. Figure 3.3.5.5 shows the normalized intensity throughout the pulse cycle. As alluded to in an earlier discussion the mechanism for populating this transition varies during the pulse cycle. During the plateau time regime the mechanism for populating the metastable level is through excitation, and this process occurs within $2 \mathrm{~mm}$ of the cathode surface. It can be seen that such emission is dominant through out the pulse cycle. During the afterpeak, metastable argon atom production arises through recombination processes, and its intensity is 20 $30 \%$ of the emission intensity maximum found during the plateau time regime.

Of these maps only the map taken at 2.3 watts demonstrates an enhancement of the metastable population at greater distances. Figure 3.3.5.6, the raw emission maps, demonstrates the relative changes in recombination processes as power is increased. 


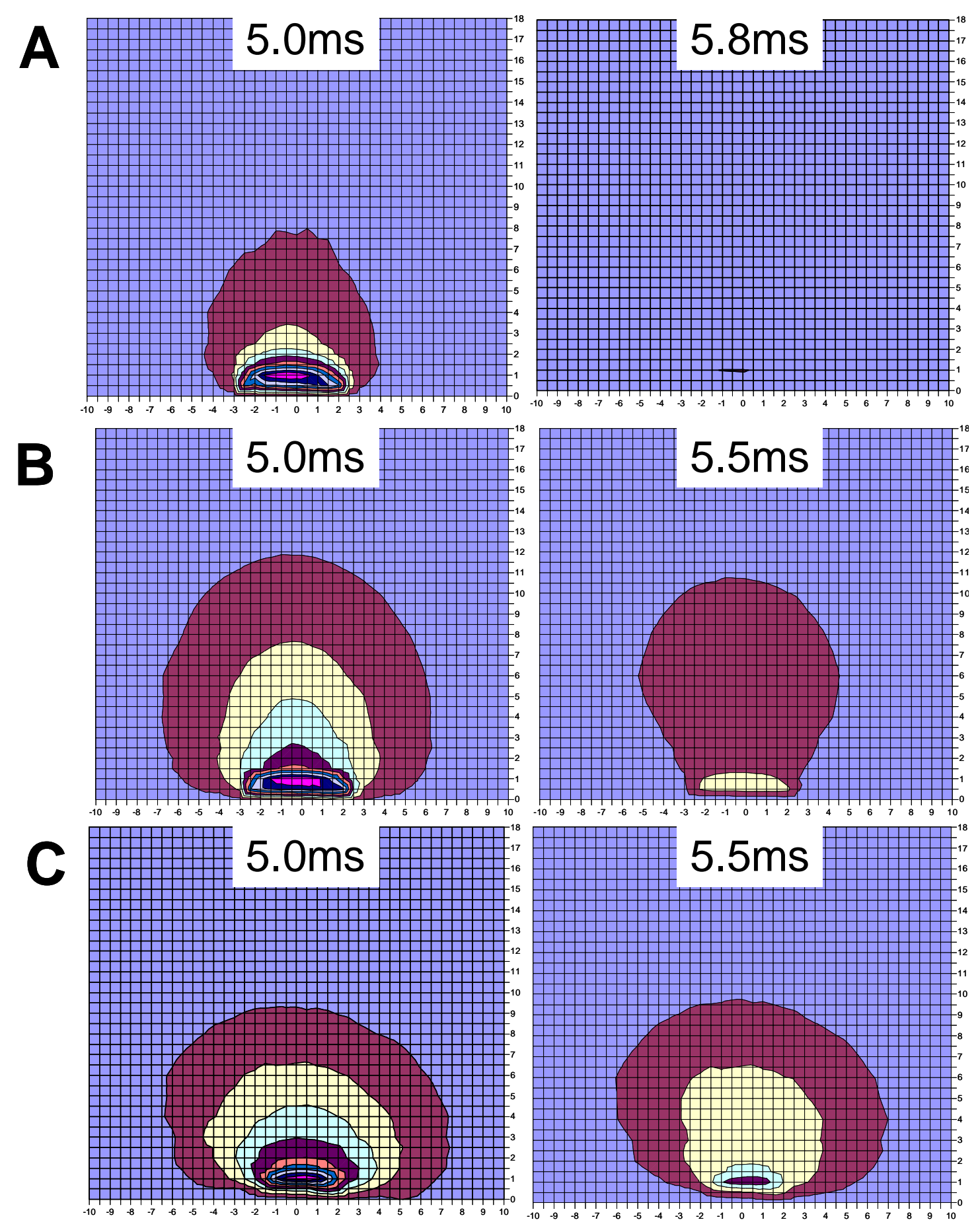

Figure 3.3.5.3 Two dimensional emission maps of metastable argon atom monitored by emission at $811.5 \mathrm{~nm}$ at a constant power of 1.5 watts and pressures of (A) 0.5 , (B) 0.8 , and (C) 1.0 torr. 


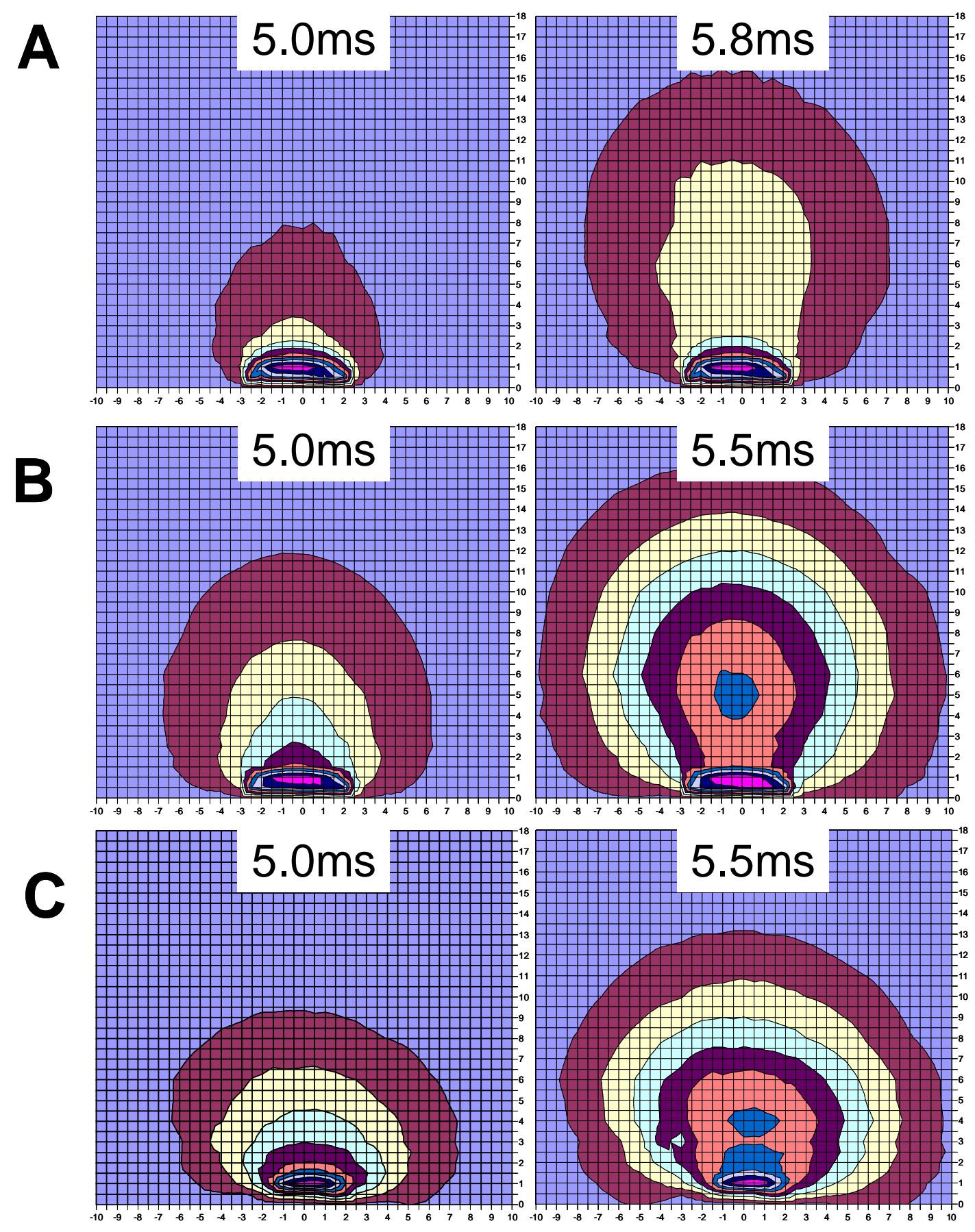

Figure 3.3.5.4 Two dimensional emission maps of metastable argon atom monitored by emission at $811.5 \mathrm{~nm}$ at a constant power of 1.5 watts and pressures of (A) 0.5 , (B) 0.8 , and (C) 1.0 torr. 

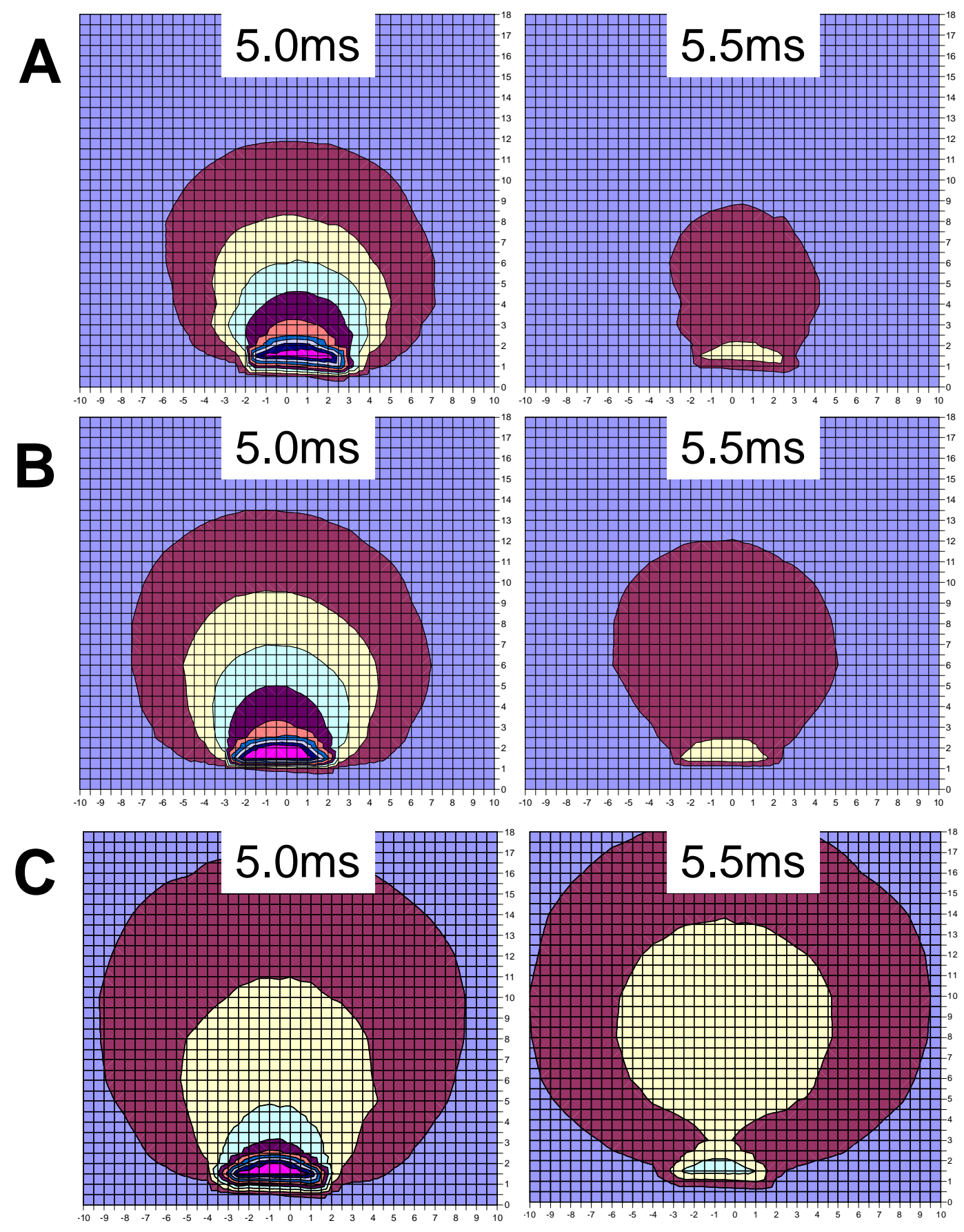

Figure 3.3.5.5 Two dimensional emission maps of argon atom metastable monitored by emission at $811.5 \mathrm{~nm}$ at a constant pressure of 0.8 torr and power of $(A) 0.5$, (B) 1.3, and (C) 2.3 watts. 

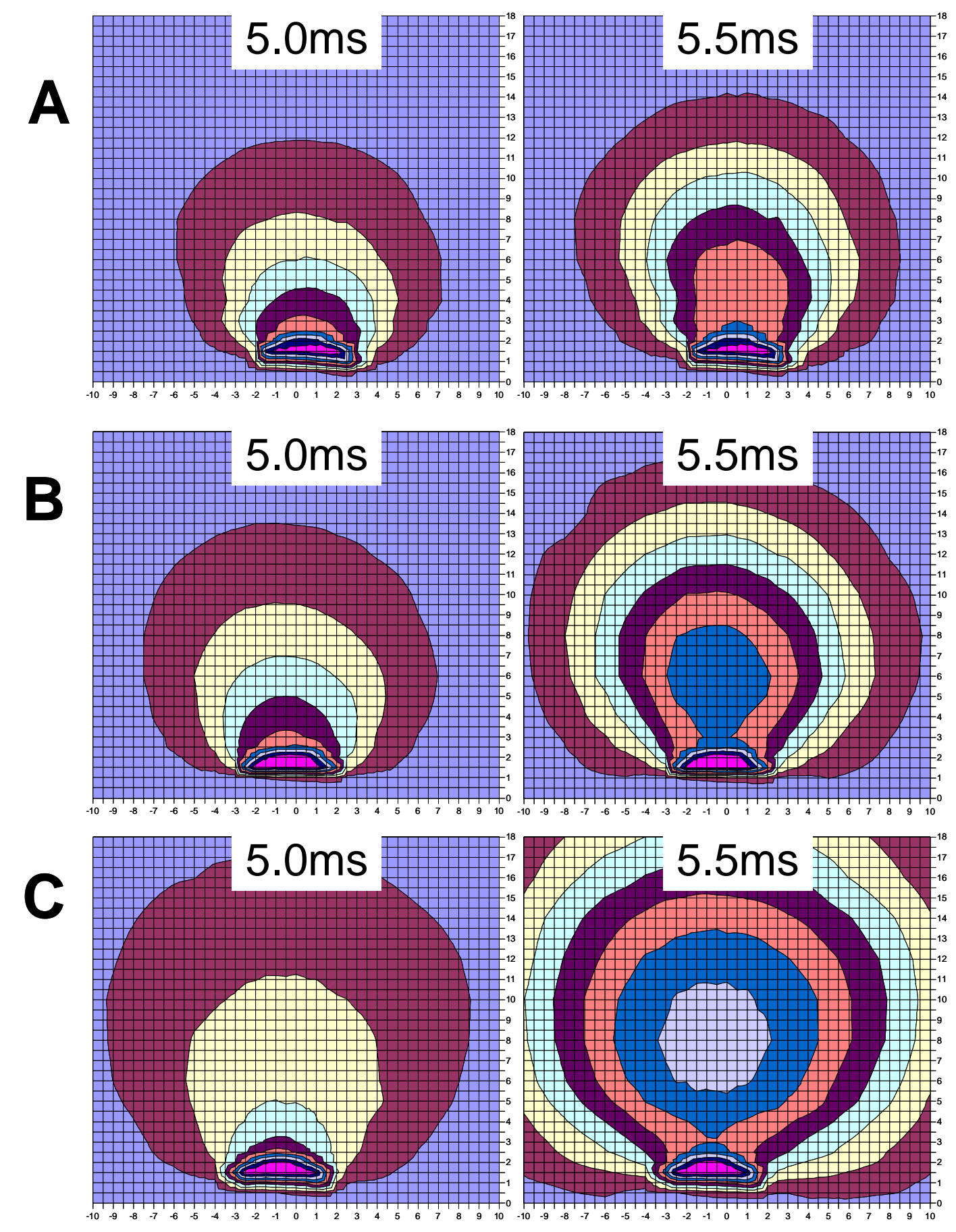

Figure 3.3.5.6 Two dimensional emission maps of argon atom metastable monitored by emission at $811.5 \mathrm{~nm}$ at a constant pressure of 0.8 torr and power of $(A) 0.5,(B) 1.3$, and $(C) 2.3$ watts. 


\subsubsection{Copper Atom States}

The following maps depict the effects of increasing pressure under constant power for a low (3.83 eV) and high (7.18 eV) energy copper atom transition. For the low energy transition two-dimensional maps are presented from the plateau, $5.0 \mathrm{~ms}$, and time at which the emission intensity has decayed to $20 \%$ of its temporal maximum during the afterpeak time regime. Maps for the high-energy transition were taken at $5.0 \mathrm{~ms}$ and temporal position at which the maximum afterpeak emission intensity occurred. At low pressures, 0.5 torr, plateau maps for both transitions demonstrate that the plasma is diffuse with electron excitation occurring $2-6 \mathrm{~mm}$ from the cathode surface. As pressure is increased the spatial maximum decreases to within $2 \mathrm{~mm}$ of the cathode surface. This shift in the spatial plateau intensity to shorter distances from the cathode surface is also followed by a confinement of the plasma emission radius. This is simply due to the decrease in the mean free path of species with the increasing pressure that results in thermalization of both sputtered atoms and electrons closer to the cathode surface.

During the afterpeak time regime this confinement effect is observed for species, Figures 3.3.5.7 and 3.3.5.8. Populating mechanisms for these two levels have been discussed in a previous section as originating from recombination of Penning ionized copper ions and electrons. The selection of monitoring the $20 \%$ decay of the low energy state was to demonstrate the significant shift in this population between plateau and afterpeak time regimes. The origin of this shift appears to be a radiative decay populating mechanism 
from high energy excited states. This $20 \%$ intensity value overlaps the spatial position of the high-energy state's afterpeak maximum position throughout each pressure change. The spatial and temporal position of the high-energy state is dictated by the formation of the metastable argon atom population discussed in the preceding section.

Increasing power increases the number of sputtered species, excitation and ionization processes, Figures 3.3.5.9 and 3.3.5.10. For the low energy transition, increasing power resulting in the emission intensity sphere increasing outward during the plateau region. This is attributed to the larger sputtered population and electron population having a kinetic energy capable of exciting this transition. Increasing the overall kinetic energy of the electron population also increases spatial and temporal position at which the $20 \%$ afterpeak maximum occurred, Figure 3.3.5.9. This is opposite of the effect noted for pressure, but is once again attributed to thermalization processes. As power is increases, a larger metastable population is available for Penning ionization. This was verified in earlier argon metastable power studies, and can be further verified by a comparing the emission ratio of this high-energy state during the plateau at $5.0 \mathrm{~ms}$ time regimes. 

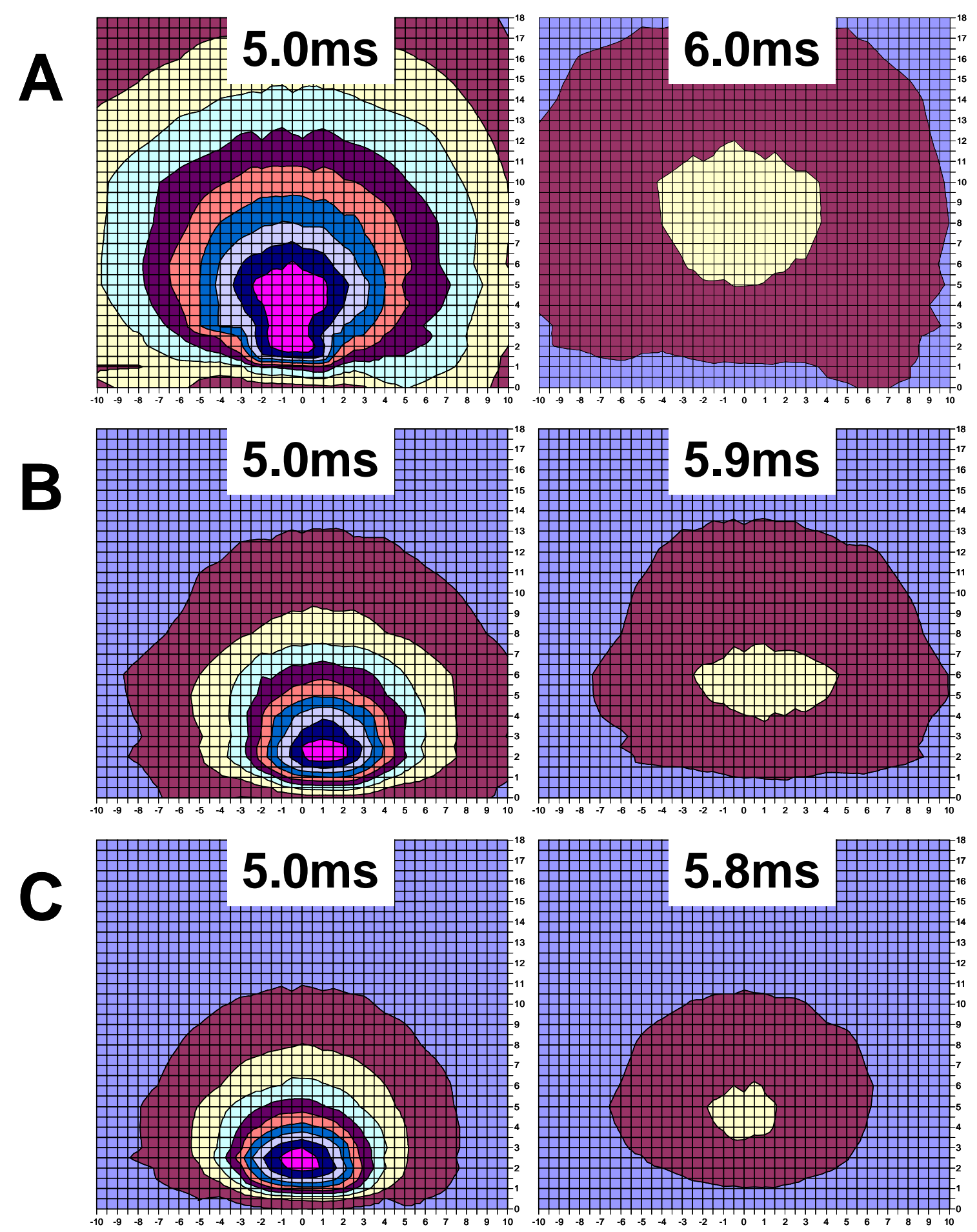

Figure 3.3.5.7 Two-dimensional emission maps of copper atom monitored by emission at $324.7 \mathrm{~nm}$ at a constant power of 1.5 watts and pressure of $(A) 0.5$, (B) 0.8 , and (C) 1.0 torr. 


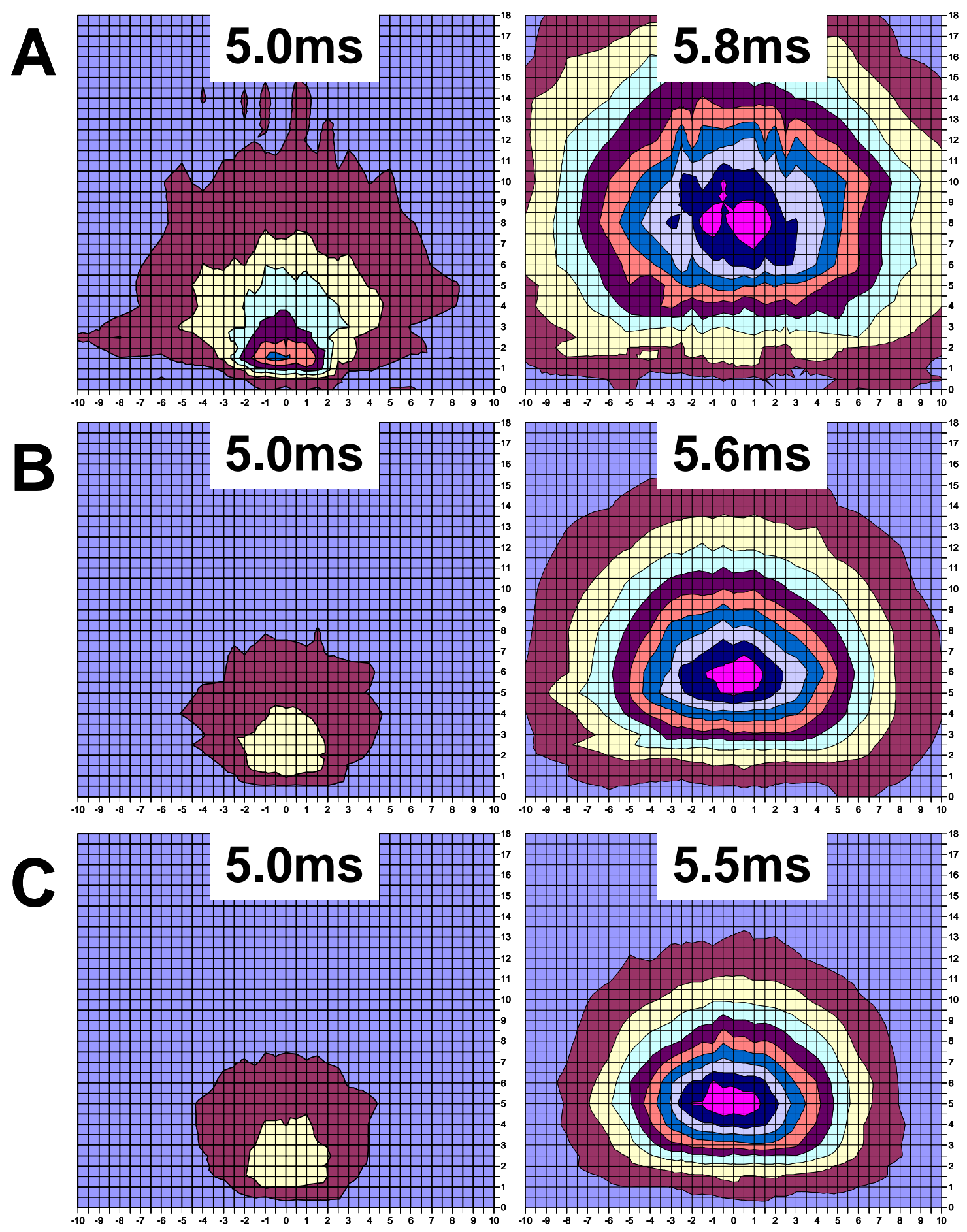

Figure 3.3.5.8 Two-dimensional emission maps of a high lying copper atom electronic state $7.18 \mathrm{eV}$ above the atom ground state, by monitoring emission at $368.7 \mathrm{~nm}$ at a constant power of 1.5 watts and pressure of $(A)$ 0.5 , (B) 0.8 , and (C) 1.0 torr. 


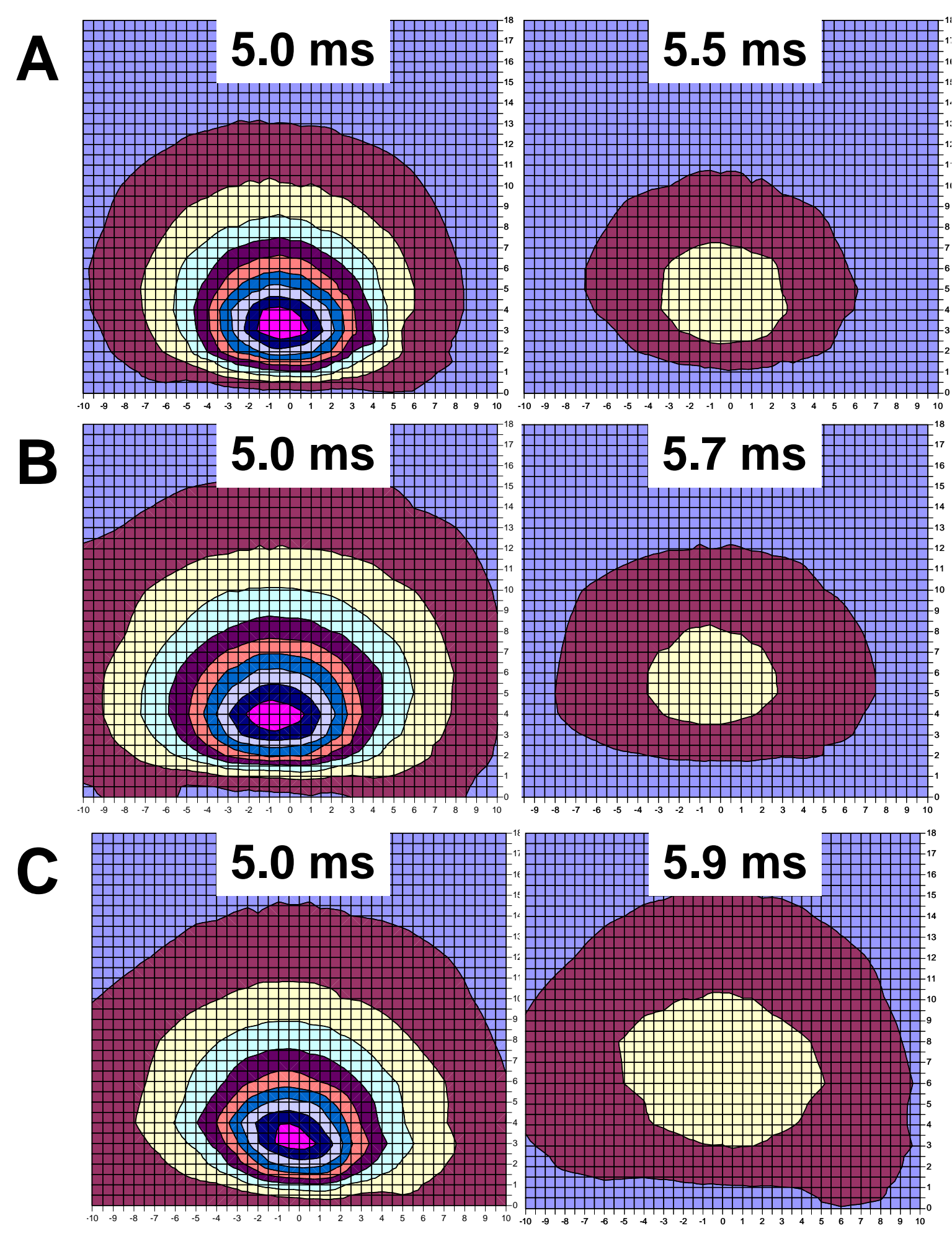

Figure.3.3.5.9 Two-dimensional emission maps of a low lying electronic transition state $3.83 \mathrm{eV}$ above the ground state monitored at $324.7 \mathrm{~nm}$, at a constant pressure of 0.8 torr and powers of $0.5,1.3$, and 2.3 watts 

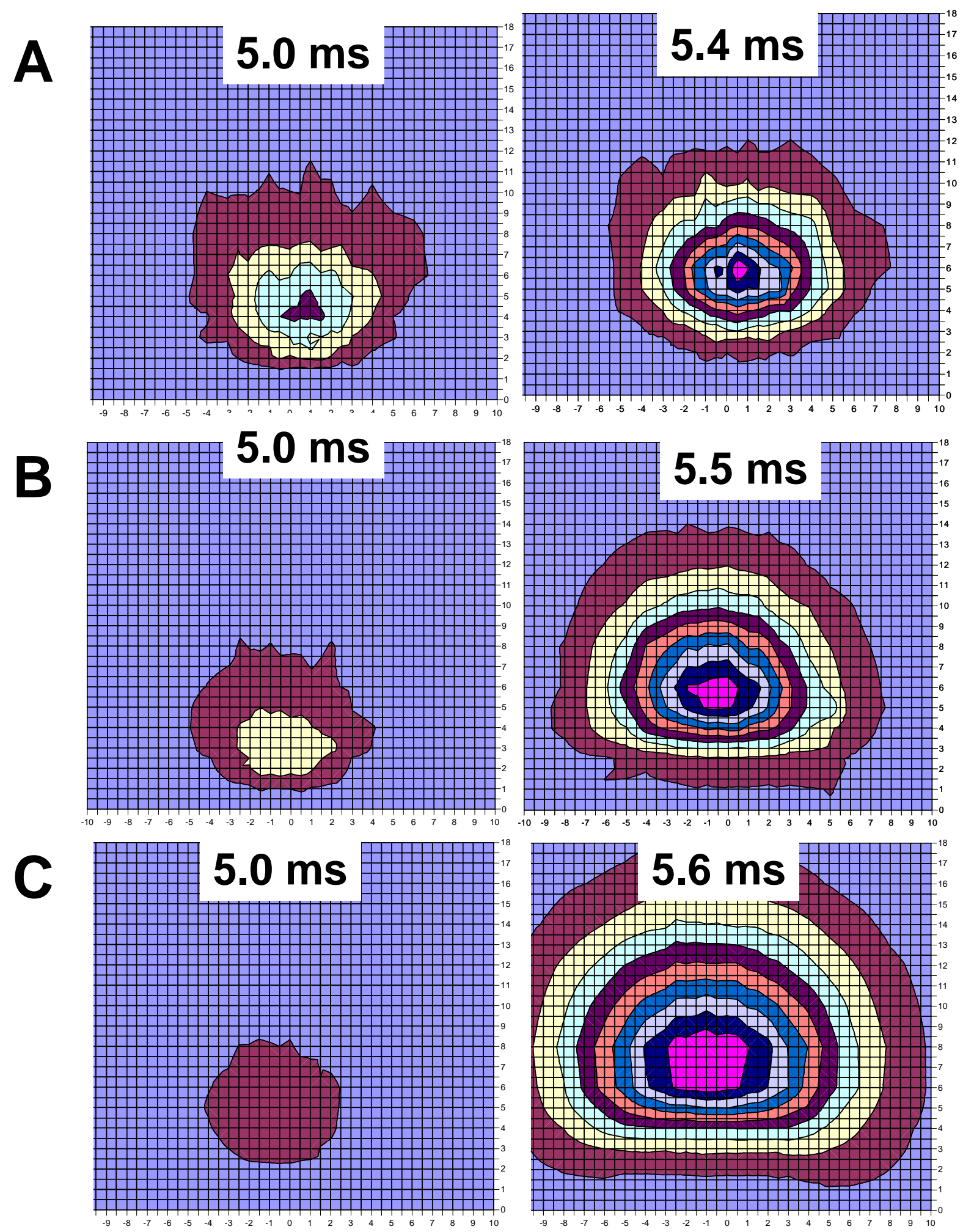

Figure 3.3.5.10 Two-dimensional emission maps of a high lying electronic transition state $7.18 \mathrm{eV}$ above the ground state monitored at $368.7 \mathrm{~nm}$, at a constant pressure of 0.8 torr and powers of $0.5,1.3$, and 2.3 watts. 


\subsubsection{Copper Ion}

Temporal copper ion emission maps were constructed by monitoring the emission at $224.7 \mathrm{~nm}$, corresponding to an electronic transition occurring $8.23 \mathrm{eV}$ above the copper ion ground state. As discussed earlier the mechanism of population for this transition is through charge exchange between a metastable argon ion and ground state copper atom. It is worth remembering at this time that unlike previous species this transition did not exhibit the characteristic plateau behavior. The emission intensity steadily climbs to its temporal maximum just before power termination at 5 to $5.1 \mathrm{~ms}$. Furthermore no afterpeak was detected for this or any other copper ion transition. The significant absence of an afterpeak for this transition strongly indicates that charge exchange does not occur once power is terminated.

Pressure maps demonstrate the relative confinement of the charge exchange process as pressure is increased, Figure 3.3.5.11. This observation is constant for every species studied as pressure increases. The most significant observation is formed in the maps representative of the afterpeak taken at 5.5 ms. Each map demonstrates a drop in intensity to $20-30 \%$ of its emission maximum. This is significant in that it does not show the characteristic temporal changes previously observed for the copper atom emission maps, $3.87 \mathrm{eV}$ and $7.18 \mathrm{eV}$. The previous copper atom emission maps demonstrated a change in temporal behavior at increasing pressure due to changes in the argon metastable atom population. Because no temporal change is detected copper ion production in the afterpeak is through Penning ionization and not charge exchange. 
Increasing power demonstrates an increase in the emission intensity for this transition, Figure 3.3.5.12. This increase is attributed to an increase in the production of the argon ion population witnessed in earlier argon ion maps, suggesting an increase in the argon ion metastable population. 

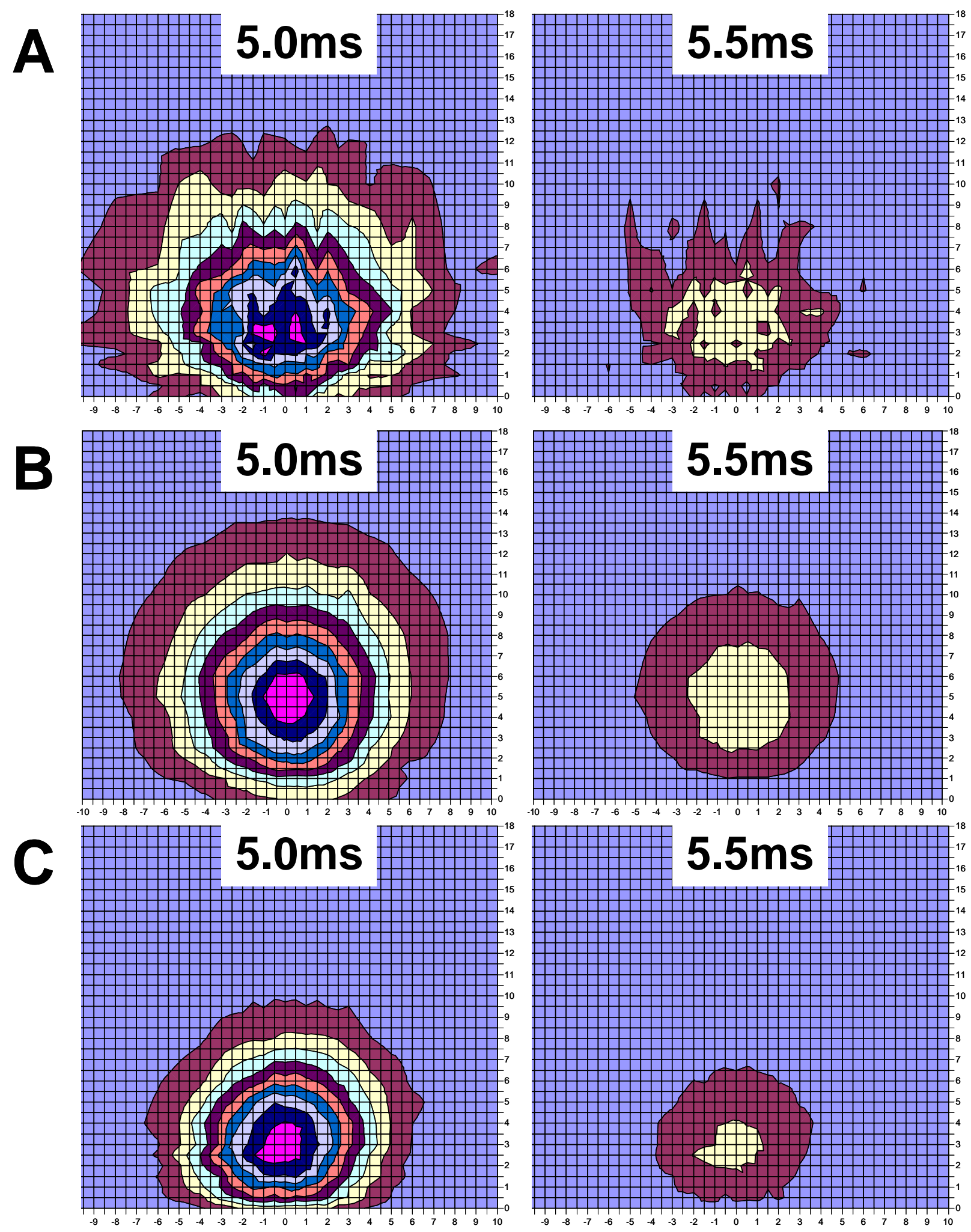

Figure 3.3.5.11 Two dimensional emission maps of copper ion emission monitored by emission at $224.7 \mathrm{~nm}$ at a constant power of 1.5 watts and pressure of $0.5,0.8$ and 1.0 torr. 

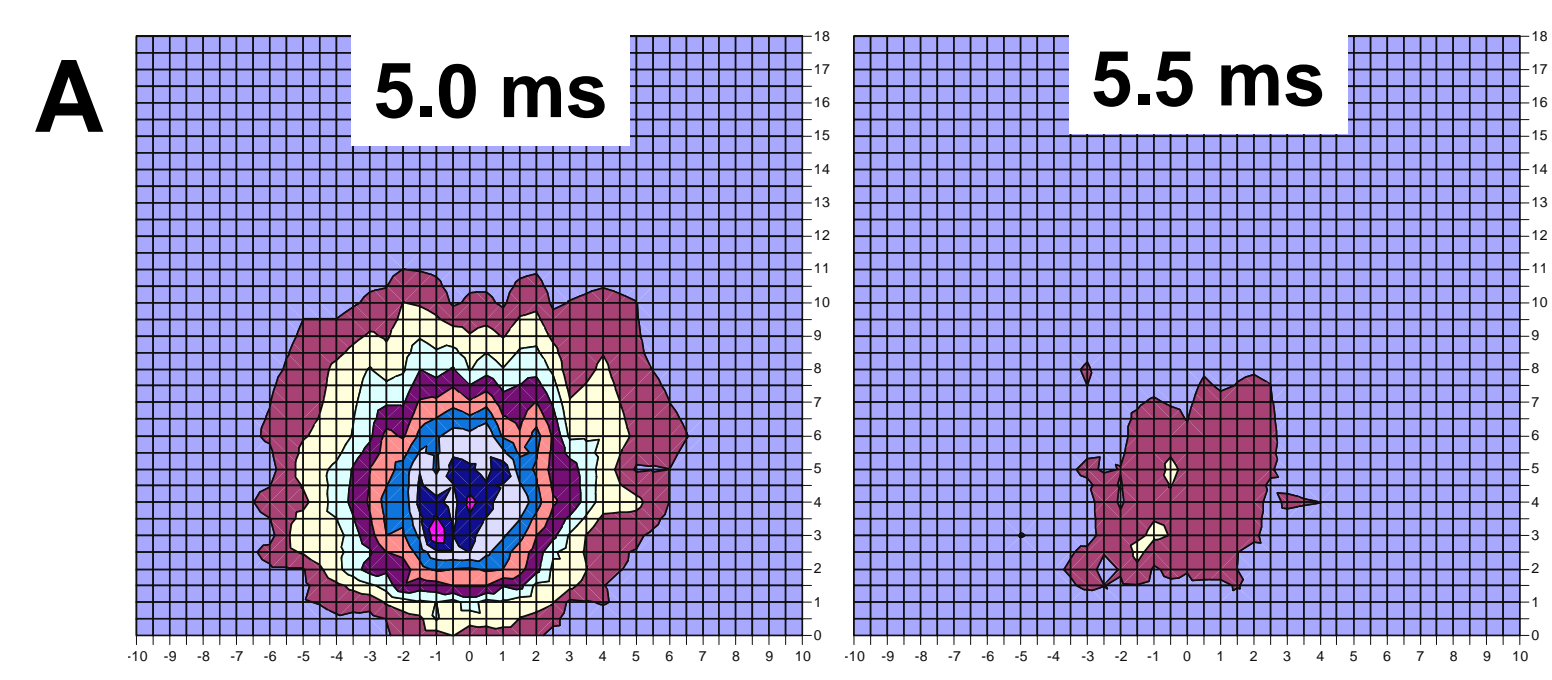

\section{B}
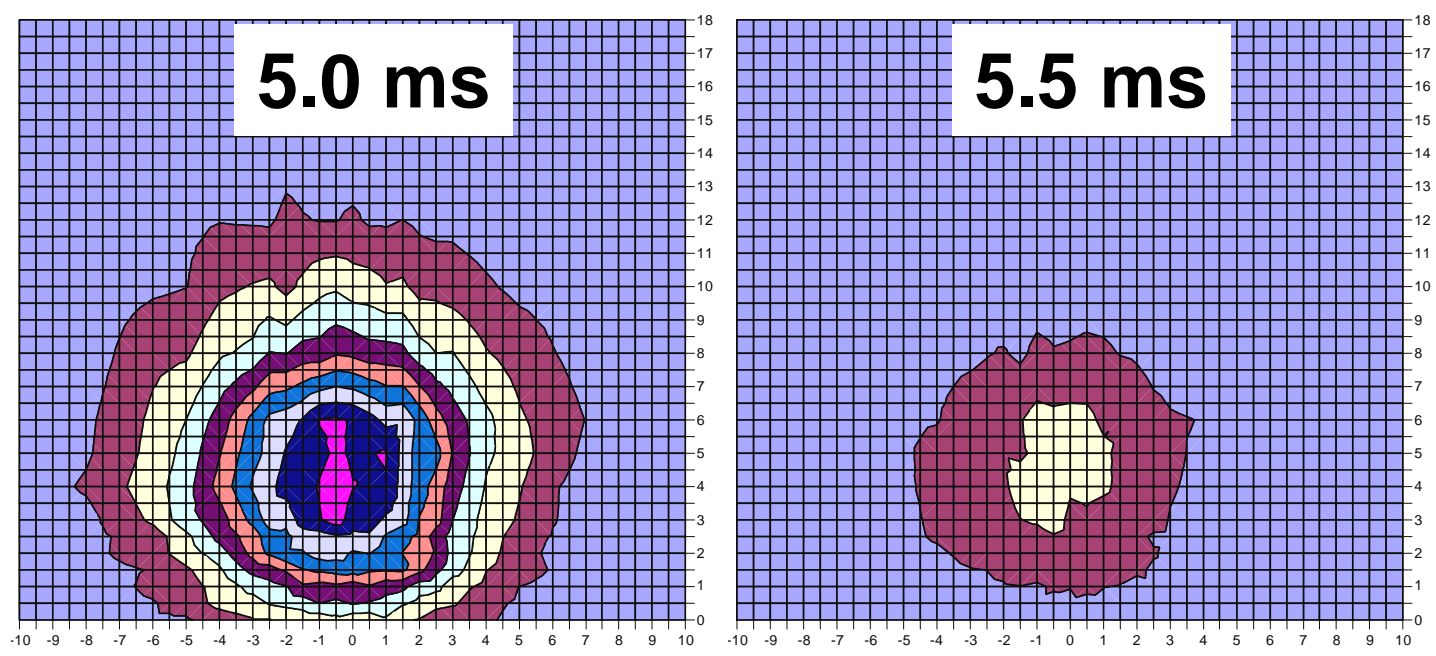

C
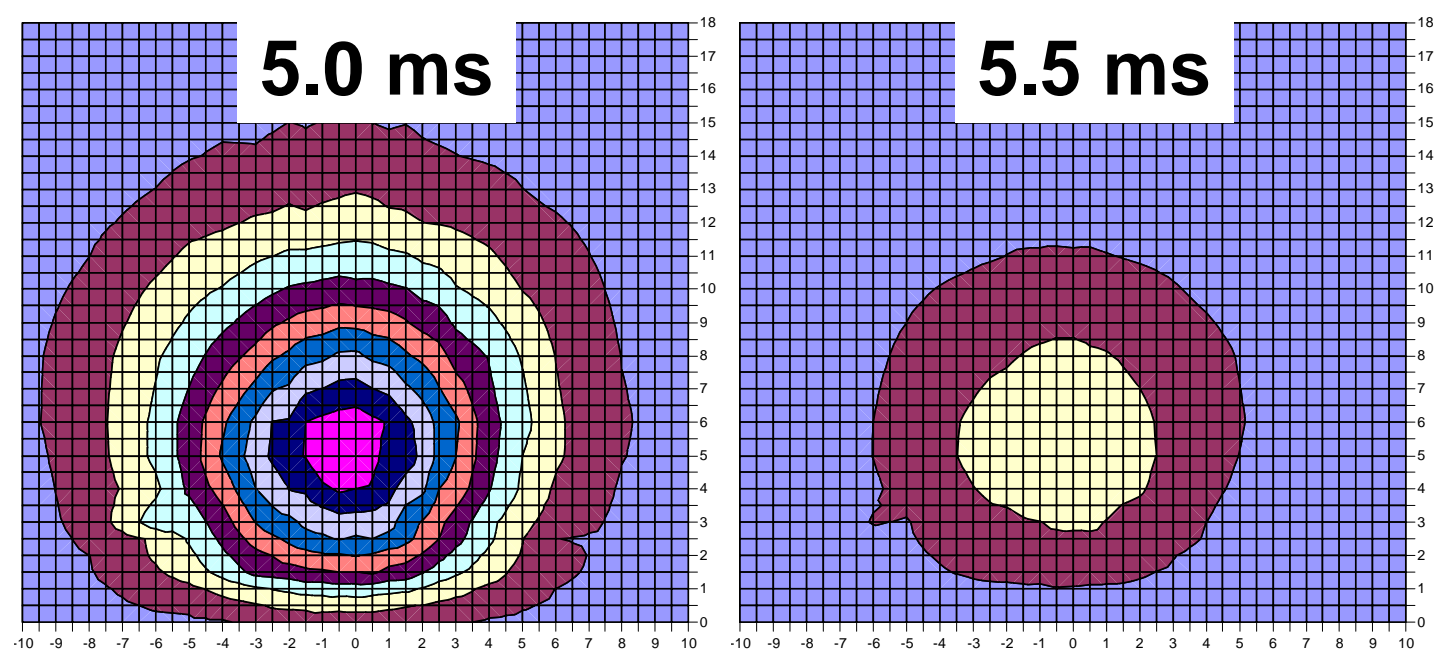

Figure 3.3.5.12 Two-dimensional maps of copper ion emission monitored at $224.7 \mathrm{~nm}$ under a constant pressure of 0.8 torr and power of $(A) 0.5,(B) 1.3$, and $(C) 2.3$ watts. 


\subsection{Conclusions}

Two-dimensional emission maps of different copper atom transitions were presented throughout the pulse cycle. These maps demonstrate that excitation and ionization processes vary both spatially and temporally through out the pulse cycle. During the plateau time regime, population of the various sputtered atom and ion electronic states is accomplished primarily by electron excitation. A discrete argon metastable population was found to maximize within $2 \mathrm{~mm}$ of the cathode surface. This population was attributed high-energy electron and fast atom excitation. Electron excitation and ionization, exhibited by argon ion, copper atom and copper ion emission was found to maximize during the plateau time regime 2 - $3 \mathrm{~mm}$ from the cathode surface. Both argon and copper ion species exhibited emission only during the plateau time regime. Charge exchange processes between the discharge gas and sputtered analyte, monitored by copper ion emission at $224.7 \mathrm{~nm}$, were found to maximize during the plateau at a distance of $3-5 \mathrm{~mm}$ from the cathode surface. Because no afterpeak was observed for these transitions it can be deduced that charge exchange does not play substantial role in the formation of copper ions during the afterpeak time regime. Once power is terminated, thermal electrons and argon ions recombine to produce metastable argon atoms. This metastable population maximized $5-7 \mathrm{~mm}$ from the cathode surface at $5.3-5.5 \mathrm{~ms}$ from the beginning of the pulse cycle. The temporal and spatial position of this metastable population coincided with the various surges in afterpeak emission intensity of high-energy copper atom electronic states. It was further deduced 
that these highly excited states decay into lower lying copper atom energy levels to yield emission. 


\section{Spectral, Spatial, and Temporal Characterization of a Millisecond Pulsed Glow Discharge: Diffusion Effects}

\subsection{Introduction}

Since its introduction as an ion source for mass spectrometry, the glow discharge has demonstrated its versatility for the direct determination of trace elements. ${ }^{35,7}$ In the last decade, GDMS investigations focused on the enhancement of analyte sensitivity and the minimization of spectral interferences. In 1989 Harrison's group proposed the use of a pulsed glow discharge ion source to achieve these improvements. ${ }^{20}$ Such pulsed plasmas afforded increases in atomization and ionization, as well as the added potential advantage of temporal discrimination between electron and Penning ionized species. Time-gated detection methods, employing measurements taken when analytical signals are at their maximum and background signals are at their minimum, permit full advantage to be taken of these pulsed glow discharge sources. ${ }^{13,17,22,36}$

It is well known that ionization in analytical glow discharge plasmas is dominated by two mechanisms; electron ionization and Penning ionization. ${ }^{35}$ Upon plasma ignition, electrons accelerate away from the cathode surface and acquire kinetic energy as they approach the anodic end of the cathode darkspace. The conversion of kinetic energy from the electron into the potential energy of the target atom through collisions leads to excitation or ionization of the atom and thermalization of the electron. This is the predominant mechanism for ionization of the discharge support gas and many molecular species that may contaminate that gas. The collision of thermalized electrons with ionized 
discharge support gas species results in the production of metastable support gas species. These metastable species possess a significant amount of potential energy. A collision between a metastable atom and a neutral analyte atom sputtered from the sample can result in the transfer of this potential energy. Sometimes this energy is sufficient to yield ionization of the analyte atom, Penning ionization. In the case of the most common discharge gas, argon, metastable energy states lie at 11.5 and $11.72 \mathrm{eV}$. This energy is sufficient to ionize the majority of elements found in the periodic table, but unable to ionize atmospheric impurity species.

The temporal response of these different ionization mechanisms has been described in great detail previously. The present research focuses on diffusion processes in pulsed glow discharges and their effect on ion signal temporal profiles. Results demonstrate that spatial discrimination between electron and Penning ionization mechanisms is possible. Variation of the plasma region sampled by mass spectrometry affords access to spatially discrete ionization regions within the plasma that can aid or enhance the temporal selectivity commonly enjoyed when using modulated glow discharge ion sources.

\subsection{Experimental}

The dc glow discharge ion source, sample introduction system, and quadrupole mass spectrometer have been described in detail. ${ }^{12}$ All data were acquired for a plasma operating at a $25 \%$ duty cycle and a frequency of $50 \mathrm{~Hz}$. Modifications of the mass spectrometer for these experiments include replacement of the $\mathrm{Cl} / \mathrm{El}$ ion source with a Bessel-box energy analyzer (Extrel 
Pittsburgh, PA), and installment of an Einzel lens assembly. The Einzel lens assembly facilitated the transmission of ions from the ion exit orifice into the Bessel-box entrance aperture. In these experiments the Bessel-box served only to focus the ion beam because its energy-filtering cone was removed to increase ion throughput. The triple quadrupole operated with the first and second quadrupoles serving as rf-only ion guides. The third quadrupole served as the mass analyzer.

Data collection was performed in three different modes. The first mode, used primarily for tuning and calibrating the mass-to-charge scale, gave the average signal over the entire pulse. This was accomplished by transferring the signal output from a continuous dynode amplifier (Galileo Electro-optic Corp., Strurbridge, MA) through a preamplifier (Combo-100, MIT, Boulder, CO) and into the computer data system (Teknivent Corp., St. Louis, MO), where the signal was averaged and integrated. In the second mode, ion temporal profile scans were digitally captured by monitoring the output of the continuous dynode with a 1-GHz oscilloscope (9370M, LeCroy, Chestnut Ridge, NY). Each captured profile is the average of 500 sweeps and contains 2500 data points. Time-gated mass spectrometric detection, the third mode, was accomplished using a boxcar integrator to collect signal through a data gate. Signal from the electron multiplier was amplified using the preamplifier, and sent to a boxcar integrator (4152, EG\&G PAR, Princeton NJ). The boxcar integrator employed a fixed gate window of $0.15 \mathrm{~ms}$ with 30 pulse profiles being averaged for each data point collected. The data gate could be positioned throughout the pulse cycle by the use of the 
digital delay generator (4144, EG\&G PAR, Princeton NJ). The output of the integrator was processed by a computer using applications software (4162, EG\&G PAR, Princeton NJ). Data collected from both the oscilloscope and boxcar integrator was then transferred into a spreadsheet (Excel 97, Microsoft Corp., USA) and then processed.

Samples were fabricated using iron powder (Analytical Grade, J.T. Baker Chemical Co.). The iron powder was pressed into sample cathode disks at 4500 psi for 2 minutes. The resultant sample disks, diameter of $0.200 "$, were mounted onto the dc direct insertion probe and introduced into the ion source chamber. The glow discharge ion source was then pumped down for two hours and preburned for one hour before any measurements were taken.

Ion optics were tuned to maximize the ${ }^{56} \mathrm{Fe}^{+}$afterpeak at a pressure of 0.5 torr and pulsed current of $2.0 \mathrm{~mA}$, and remained constant for all other pressures and currents. Probe position was varied in $2 \mathrm{~mm}$ increments spanning a sampling region of $2-20 \mathrm{~mm}$ at various current and pressure levels. Ion temporal profiles were captured digitally for two species, $\mathrm{Ar}^{+}$and $\mathrm{Fe}^{+}$, representing electron ionization and Penning ionization respectively. 


\subsection{Results and Discussion}

\subsubsection{Sampling Region}

Immediately following the application of power in the pulsed glow discharge, species present in the gas phase are subjected to electron ionization. Typical ion signal temporal profiles of such species exhibit what is known as a prepeak.

Figure 4.3.1.1 illustrates the effect of sampling distance on the ion signal profiles for the electron ionized species, ${ }^{40} \mathrm{Ar}^{+}$. At shortest distance, $2 \mathrm{~mm}$, there is no prepeak present and the overall argon ion intensity is low. At this distance ions are essentially sampled inside the cathode darkspace. Inside the cathode darkspace the electron ionization cross section is low, the kinetic energy of electrons is too high to favor ionization. As distance increases both the prepeak and plateau signal intensities rise, as the cross section for electron ionization increases. The increase in ionization is due to the loss of kinetic energy of electrons. This loss of kinetic energy is attributed to the decline in electrostatic repulsion at increasing distances from the negative biased cathode, and in thermalization effects brought on by collisions with atomic species. What appears to be a small afterpeak exists for argon is actually a ringing effect of the pulsed power supply. Monitoring the pulse output with a high voltage probe also reveals this ringing artifact. As sampling distance increases further, the prepeak subsides, whereas the plateau intensities rise. Competition now exists between electron ionization and electron-ion recombination. Increases in the plateau convey that there are sufficient numbers of electrons with enough energy to yield ionization; however, the decline and absence of the prepeak also conveys that 

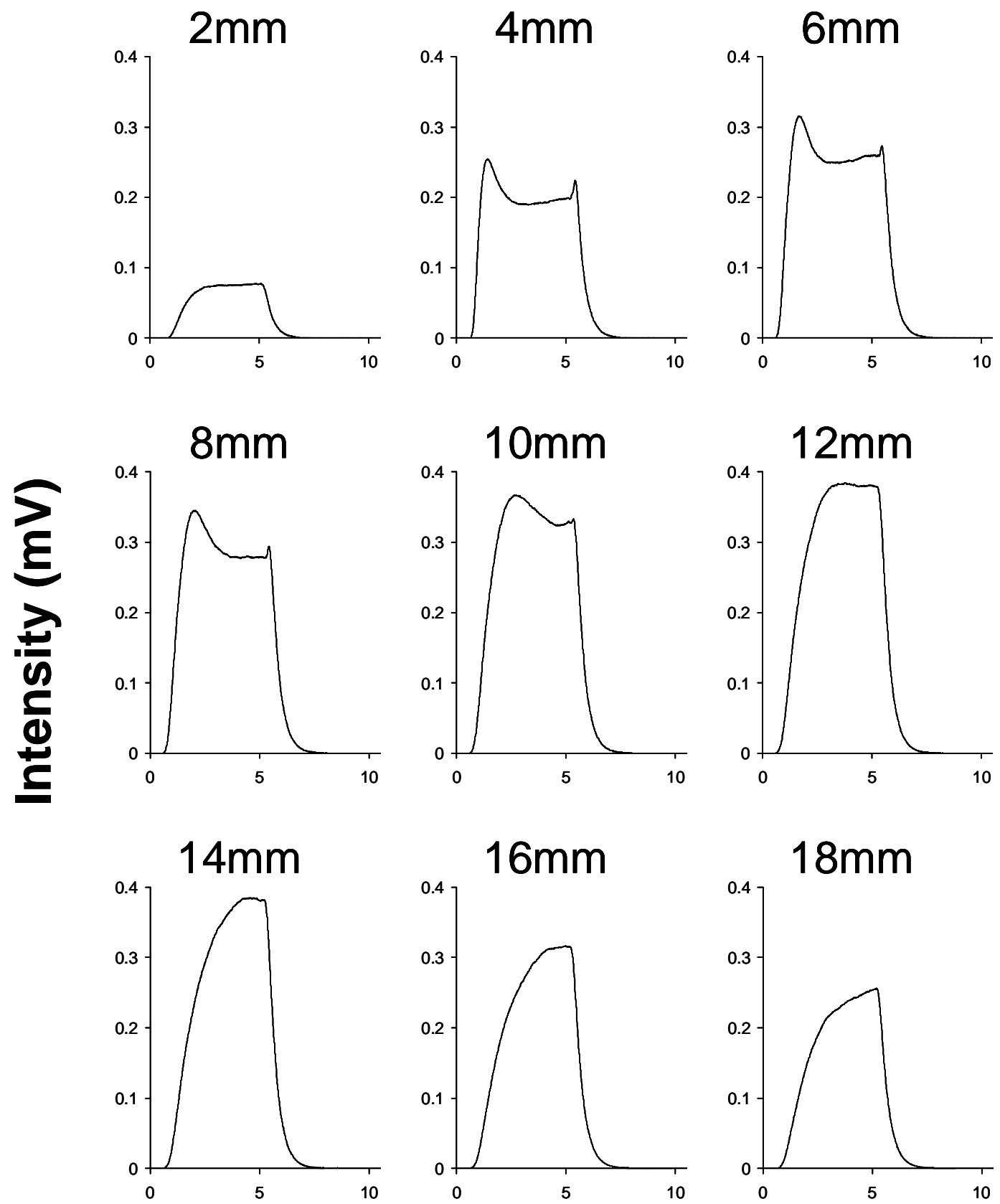

\section{Time ms}

Figure 4.3.1.1 Temporal response of ${ }^{40} \mathrm{Ar}^{+}$at increasing distances under a constant pressure of 0.5 torr and pulsed current of $2.0 \mathrm{~mA}$. 
there is a sufficient population of thermalized electrons present for recombination to become significant. At $10 \mathrm{~mm}$ from the cathode the plateau intensity peaks. At distances beyond $10 \mathrm{~mm}$, signal intensity declines. At these larger distances only a small population of electrons has sufficient energy to ionize argon atoms, while asymmetric charge transfer and metastable recombination processes deplete the argon ion population. As distance was increased, a very minute temporal delay for the onset of the plateau was observed. This delay represents the time that is necessary for electrons to diffuse from the cathode to the ion exit orifice where ionization takes place. The exact sampling position at which these maxima will occur depends upon discharge pressure, current and voltage. These variables dictate the number densities and kinetic energies of electrons, argon ions and sputtered atoms.

Similar behavior is observed for the Penning ionized species, ${ }^{56} \mathrm{Fe}^{+}$, under similar discharge conditions, Figure 4.3.1.2. At very short sampling distances, the ionized sputtered species exhibit minimal signal. This is a result of the low cross section for ionization as discussed above for argon. The absence of any afterpeak indicates the absence of significant Penning ionization in this area. As the observation distance between the cathode and ion exit orifice increases, the afterpeak intensity rises and then maximizes. This maximum occurs a few millimeters beyond the ${ }^{40} \mathrm{Ar}^{+}$maximum depending on discharge parameters (current/pressure). If the afterpeak is a result of Penning ionization, the spatial distribution of the metastable population explains this behavior. As discussed previously, metastable atoms are formed through the recombination of thermal 

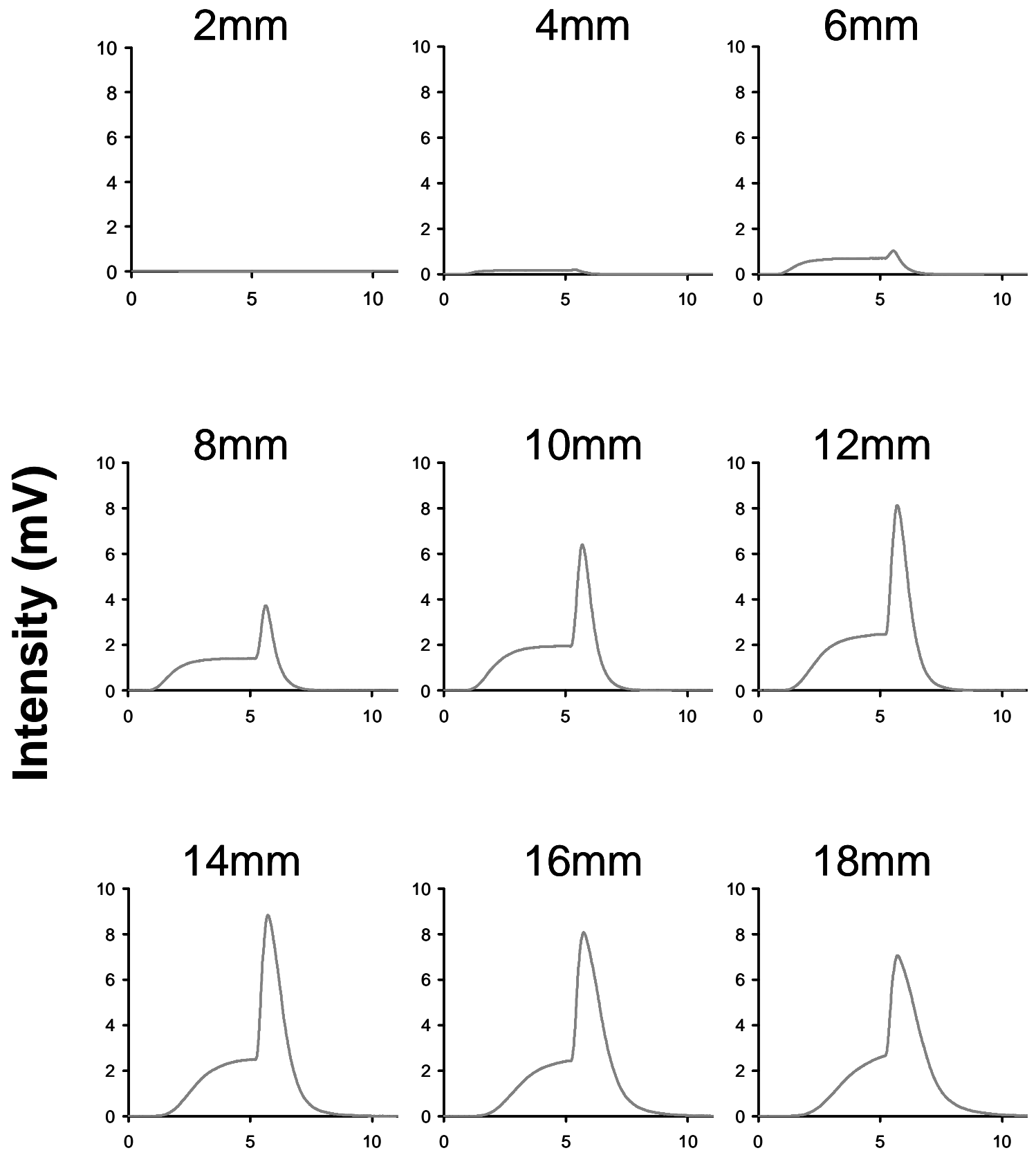

\section{Time ms}

Figure 4.3.1.2 Temporal response of ${ }^{56} \mathrm{Fe}^{+}$at increasing distances at under a constant pressure of 0.5 torr and pulsed current of $2.0 \mathrm{~mA}$. 
electrons and argon ions. At close distances, a large part of the electron population has kinetic energies that are too great to favor recombination. At increasing distances electrons thermalize through elastic collisions and recombine with argon ions to form metastable atoms. At these increasing distances, the afterpeak also exhibits a large tailing effect. As the diffusion distance increases, sputtered atoms and argon metastables are subjected to a larger number of elastic collisions during their journey to the ion exit orifice. This large spatial distribution of metastables and sputtered neutrals results in ionization well after power is terminated.

\subsubsection{Spatial Discrimination}

Mass spectrometric analysis is based on the sampling of ions formed near or at the ion exit orifice. Different ionization regions are accessed by varying the distance of the probe with respect to the ion exit orifice. Figure 4.3.2.1 shows the overlapping ion signal temporal profiles for electron ionized discharge gas species, ${ }^{40} \mathrm{Ar}^{+}$, and penning ionized sputtered species,${ }^{56} \mathrm{Fe}^{+}$, at increasing distance under constant pressure of 0.5 torr and a pulsed current of $2.0 \mathrm{~mA}$. As the sampling distance increases, both species reach maxima and then decay. The distance at which each maximum will occur depends on the applied potential and pressure of the discharge gas. The electron ionized ${ }^{40} \mathrm{Ar}^{+}$maximizes first at a sampling distance of $6 \mathrm{~mm}$, this distance represents the point in the plasma with the highest cross section for electron ionization. In contrast, the Penning ionized ${ }^{56} \mathrm{Fe}^{+}$maximizes at $14 \mathrm{~mm}, 8 \mathrm{~mm}$ beyond the maxima for electron ionization. Once again electrons must cool, allowing for their recombination with 
argon ions to occur, and resulting in the formation of argon metastables. Further information is gained from the actual shape of each temporal profile arising by electron and Penning ionization.

As distance increases the predominant change for the electron-ionized ion temporal profile is its intensity. The onset and decay of each ${ }^{40} \mathrm{Ar}^{+}$profile shows only minor delays and lags from the actual pulse power profile. The characteristic prepeak is observed only at certain distances close to the ion exit orifice. In contrast the Penning ionized species ${ }^{56} \mathrm{Fe}^{+}$, shows considerable effects ascribed to diffusion at increasing distances. Increasing the distance increases the time necessary for sputtered atoms to diffuse from the cathode to the ion exit orifice, thus generating the observed lag. With increasing distance, the onset of the afterpeak appears at later; however, it also broadens indicating greater diffusion. The large tailing of the afterpeak is explained by the large spatial window of metastables and sputtered atoms. The figures demonstrate that at greater distances diffusion and spatial separation increase. 


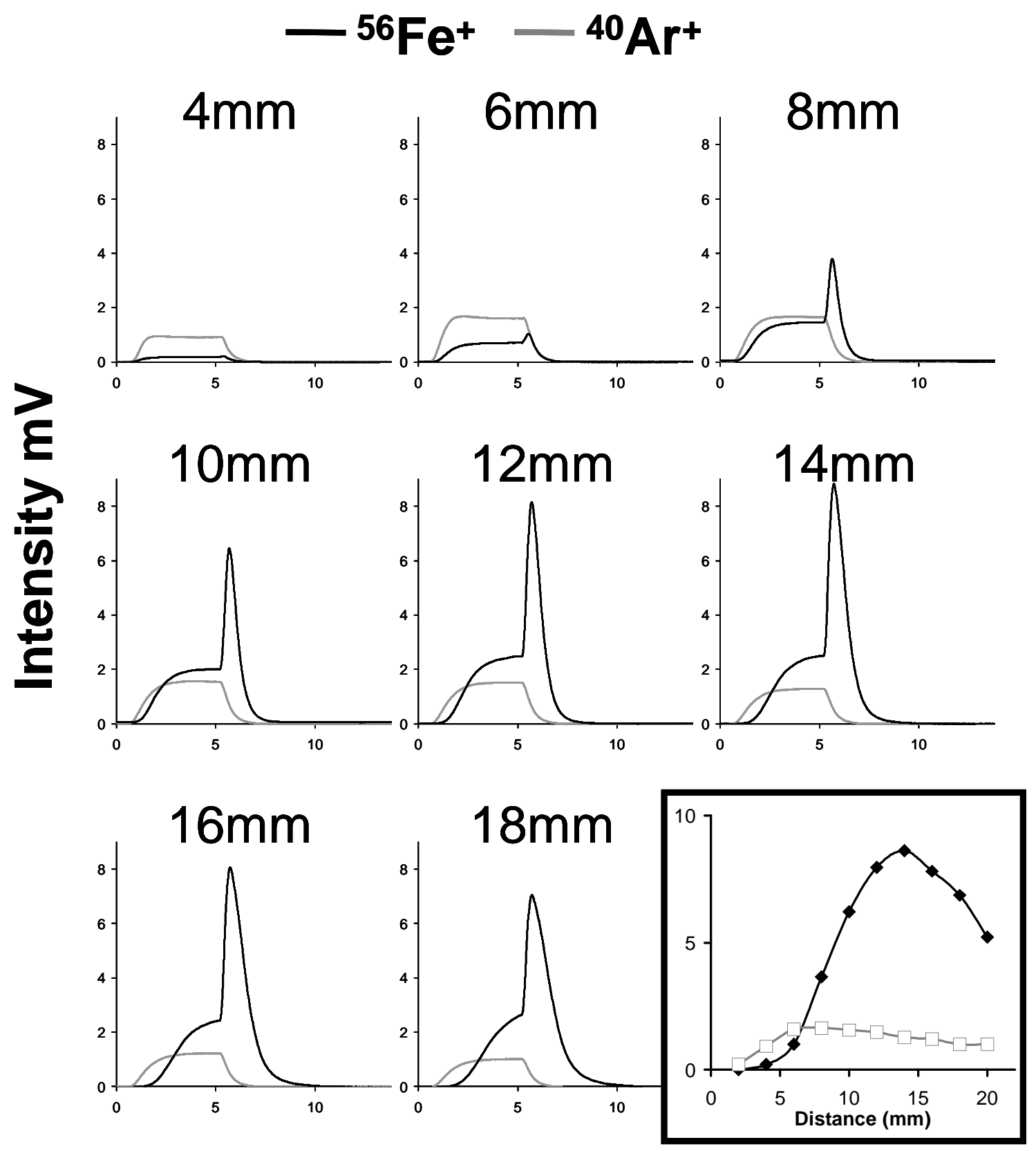

ms

Figure 4.3.2.1 Overlapping ion temporal profiles of ${ }^{40} \mathrm{Ar}^{+}$and ${ }^{56} \mathrm{Fe}^{+}$at increasing distances at 0.5 torr and $2.0 \mathrm{~mA}$. Also included is a plot of maxim intensity of each as function of distance. 


\subsubsection{Pressure Effects}

Variations in pressure change both ionization and sputtering processes. Glow discharges closely follow Ohm's law, with gas pressure defining the conductivity between the two electrodes. As the pressure is increased, there are more particles present to be charge carriers allowing higher currents to flow at lower voltages. As the voltage decreases, the kinetic energy of argon ions that impact the cathode's surface also decreases leading to a smaller sputtered atom population. As pressure increases the position of the sputtered ion afterpeak maximizes closer to the ion exit orifice. Once again, the afterpeak behavior can be explained by considering the factors that result in metastable atom production. Pressure defines the collision frequencies and the mean free paths of particles within the plasma. Working at constant current, a decrease in the pressure requires an increase in the applied voltage to sustain this current level. This results in an increase in the kinetic energy of charged species. This fact coupled with a lower collision frequency implies that electrons must travel farther into the negative glow region before they are thermalized. The following figures illustrate the overlapping ion temporal profiles of ${ }^{40} \mathrm{Ar}^{+}$and ${ }^{56} \mathrm{Fe}^{+}$at 0.5 and 0.8 torr at a constant pulsed current of $2.0 \mathrm{~mA}$. Adjacent to the ion profiles are plots of plateau and afterpeak intensities with increasing distance.

As the pressure is increased the afterpeak maximizes at distances closer to the cathode surface, Figures 4.3.3.1 A and B. This is a result of the increased collision frequency and electron cooling mechanism noted for metastable production. Increases in pressure result in a decrease in signal intensity of 

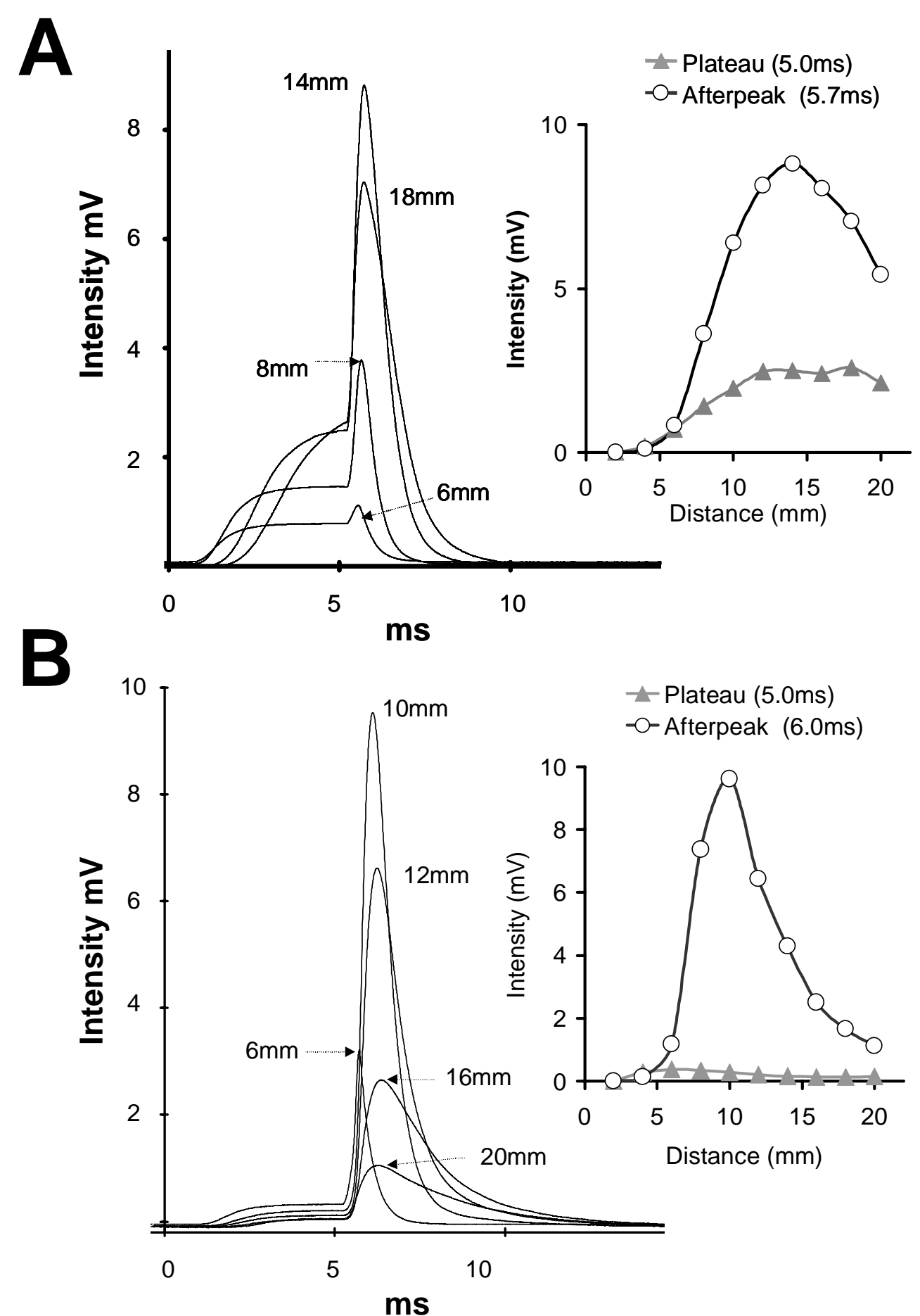

Figure 4.3.3.1 Overlapping ion temporal profiles of ${ }^{56} \mathrm{Fe}^{+}$at increasing distances under a constant current of $2.0 \mathrm{~mA}$ using a pressure of $A$ ) 0.5 torr and B) 0.8 torr. Inlaid in each is a plot of plateau intensity taken at $5.0 \mathrm{~ms}$ and afterpeak intensity $(6.0 \mathrm{~ms})$ with increasing sampling distance. 

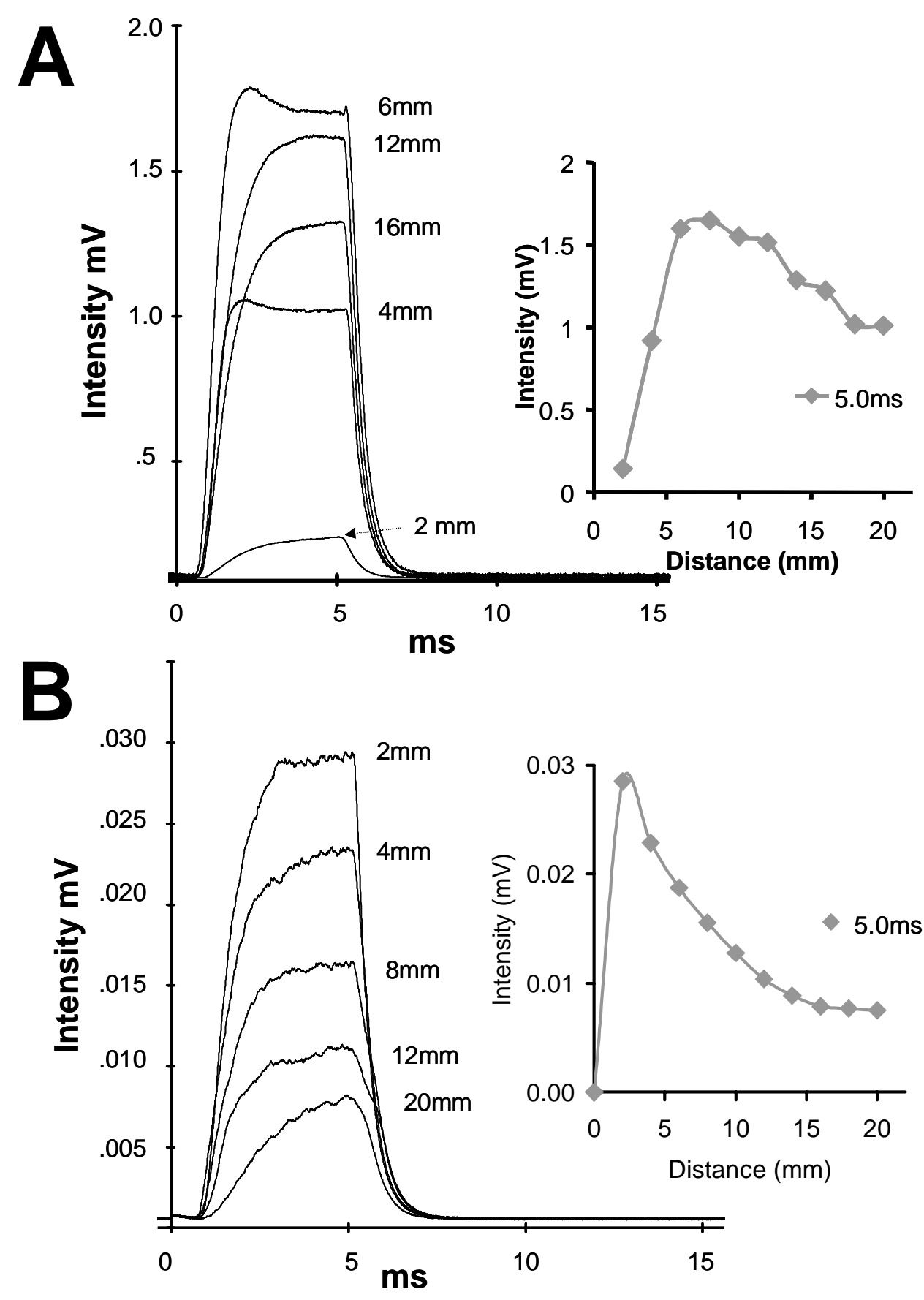

Figure 4.3.3.2 Overlapping ion temporal profiles of ${ }^{40} \mathrm{Ar}^{+}$at increasing distances under a constant current of $2.0 \mathrm{~mA}$ using a pressure of A) 0.5 torr and B) 0.8 torr. Inlaid in each is a plot of plateau intensity with increasing sampling distance. 
the electron impact species ${ }^{40} \mathrm{Ar}^{+}$, Figures 4.3.3.2 $\mathrm{A}$ and $\mathrm{B}$. At higher pressures, electrons are thermalized closer to the cathode owing to the reduction in mean free path. Once thermalized, electrons readily recombine with argon ions to form metastable argon atoms. Because electrons thermalize at shorter distances, the metastable argon atom population also maximizes at shorter distances from the cathode. As the pressure of the discharge gas increases the collisions frequency increases, electrons cool closer to the cathode surface, decreasing the size of the zone of electron ionization.

\subsubsection{Time-Gated Detection}

Time gated detection methods afford discrimination between the electron ionized and Penning ionized species in modulated glow discharges. The following time-gated spectra illustrate the temporal and spatial discrimination between the electron ionized species argon and the ionized sputtered analyte, Figure 4.3.4.1. The plasma was sampled at a distance of $16 \mathrm{~mm}$, a pressure of 0.8 torr, and a pulsed current of $2.0 \mathrm{~mA}$. The ion signal temporal profile and data gate used to collect each spectrum are visible. Note the intensity of the electron ionized species, ${ }^{40} \mathrm{Ar}^{+}$, and sputtered species, ${ }^{56} \mathrm{Fe}^{+}$, under these conditions electron ionization is discriminated by pressure, temporal and spatial considerations. Under higher pressures only a small population of electrons which reach the ion exit orifice are capable of electron ionization. Sampling at a large distance, as previously demonstrated, further reduces the signal intensity of 
the argon ion. A temporal delay was positioned which affords the complete discrimination against electron ionized ${ }^{40} \mathrm{Ar}^{+}$.

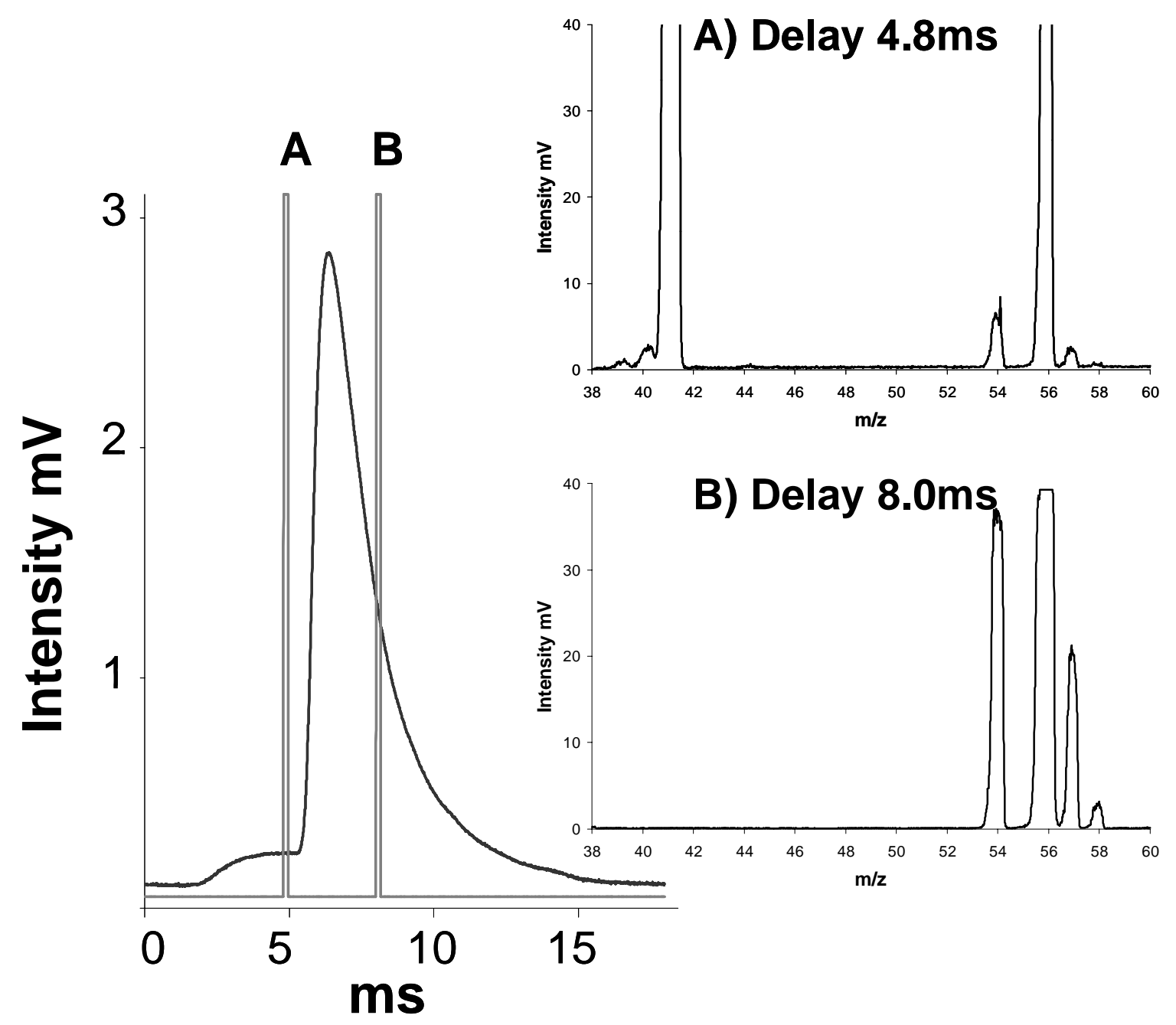

Figure 4.3.4.1 Time-gated detection of a pure iron sample was acquired by positioning a data gate $4.8 \mathrm{~ms}(\mathbf{A})$, plateau, and at $8.0 \mathrm{~ms}(\mathbf{B})$, afterpeak on the ${ }^{56} \mathrm{Fe}^{+}$ion profile. Using a sampling distance of $16 \mathrm{~mm}$, discharge gas pressure of 0.8 torr, and a pulsed current of $2.0 \mathrm{~mA}$. 
Limits, however, do exist when using spatial discrimination to assist in time gated measurements. Figure 4.3.4.2. shows data indicating that under certain sampling conditions, the afterpeak has not decayed to baseline before the onset of the next pulse cycle. The analysis utility of time-gated detection relies on each measurement being unaffected by the pulse population.

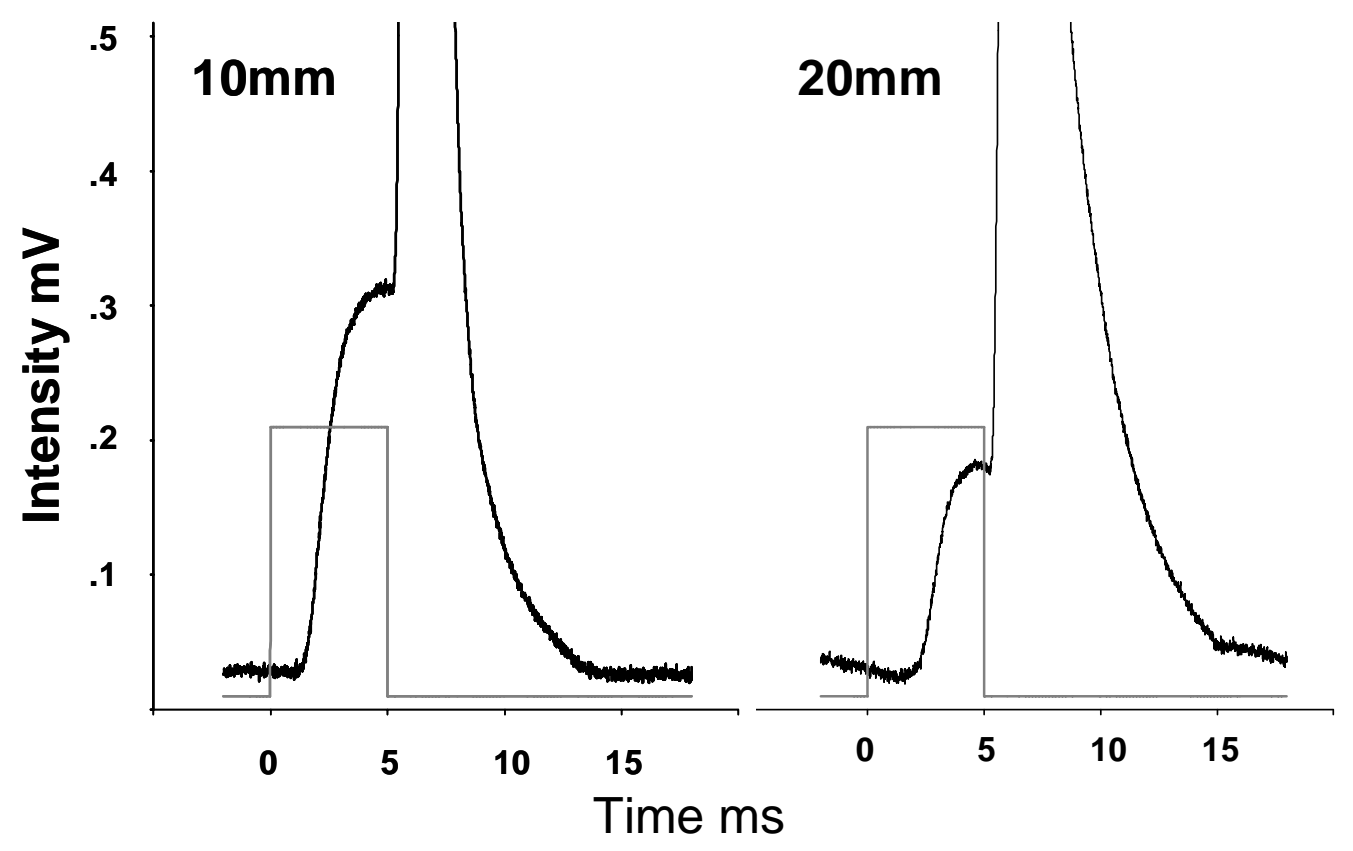

Figure 4.3.4.2 Temporal response of ${ }^{56} \mathrm{Fe}^{+}$and the applied power pulse at a sampling distance of $10 \mathrm{~mm}$ and $20 \mathrm{~mm}$ at a constant pressure of 0.8 torr and pulsed current of $2.0 \mathrm{~mA}$. 


\subsection{Conclusions}

Sampling ions at further form the ion exit orifice shows considerable differences between electron and Penning ionized species. Spatial regions of Penning ionization exist that are essentially free of election ionization.

Discrimination against electron-ionized species is approached from two different directions temporal and spatial. The analytical utility of this approach is readily achieved through the use of time gated detection methods. Future investigations will center on discrimination of ionization mechanisms based on kinetic energy distributions. Previous investigators have demonstrated that such discrimination is possible using steady state dc and rf glow discharges. ${ }^{37,38,39}$ By tailoring experimental parameters such as pressure, power, temporal response, and sampling distance; time-gated detection should permit in the ready removal of signal due to electron ionized species. 


\section{Temporal Separation of ${ }^{40} \mathrm{Ca}^{+}$from ${ }^{40} \mathrm{Ar}^{+}$in Time Gated Glow Discharge Using Mass Spectrometry}

\subsection{Introduction}

Glow discharge mass spectrometry (GDMS) is a proven tool for the direct determination of trace elements in a variety of solid state materials. Glow discharges came to the attention of analysts in the late 1960s. At that time glow discharges began to be employed as emission sources for the trace elemental determination of solid samples. ${ }^{15}$ The universal utility of the glow discharge as an atomization/excitation/ionization source has been well documented. The use of GDMS in the determination of trace elements is often limited by the existence of isobaric interferences. Isobaric interferences arise from atomic or molecular species having the same nominal mass to charge ratio as the analyte of interest. Sources of these interferences are the host matrix, the discharge gas species, or molecular species formed by discharge gas-sputtered atom interactions. ${ }^{40}$ An example of such discharge gas related isobaric interference arises in the detection of calcium in argon plasmas. Both major argon and calcium isotopes are found at a nominal $\mathrm{m} / \mathrm{z}$ of 40 . The separation of these isotopes requires a mass spectrometer to operate at levels of resolution in excess of 190,000 . Typically, such a determination relies on the use of the next most abundant isotope free from any spectral interference. For the determination of calcium, use of the less abundant ${ }^{44} \mathrm{Ca}^{+}$isotope results in a 45 fold loss in sensitivity because of its lower abundance relative to the ${ }^{40} \mathrm{Ca}^{+}$isotope. Additionally, this isotope frequently suffers isobaric interference from ${ }^{12} \mathrm{C}^{16} \mathrm{O}_{2}{ }^{+}$. 
An alternate approach to the determination of calcium would involve the elimination of argon by changing the discharge gas. However, changes in the support gas also lead to changes in atomization and ionization. The use of a gas having a higher mass leads to a higher sputtering yield, but also leads to a larger number of interferences due to the larger number of isotopes involved. ${ }^{41}$ Additionally, higher mass gases have lower ionization potentials that result in decreased sensitivity for the higher ionization potential elements.

Steady state discharges rely on the application of a constant potential to the cathode. As ions and high-energy neutrals bombard the sample, the cathode begins to heat. Such heating leads to a higher resistance and an annealing effect that, in turn, leads to sample cracking, discharge arcing, or the preferential sputtering of the sample matrix. ${ }^{11}$ Methods to alleviate heating in steady state discharges are limited to operation at lower potentials, changes in cathode geometry, or water-cooling of the cathode. An alternative to operation with a continuous, steady potential is operation with a pulsed, or modulated, potential. By applying potentials in cycles consisting of short on pulses, followed by longer off times, the cathode is allowed to cool and higher instantaneous voltages and currents can be applied. Higher instantaneous voltages and currents increase the sputtering yield and analyte excitation/ionization.

Typical pulsed glow discharge plasmas employ a square wave potential. Current work in this laboratory employs a $25 \%$ duty cycle consisting of a $5 \mathrm{~ms}$ of applied power, on-time, followed by $15 \mathrm{~ms}$ of no applied power, off-time. During the $5 \mathrm{~ms}$ of on-time sputtering and excitation/ionization processes evolve to mimic 
steady state discharges. In the $15 \mathrm{~ms}$ off-time that follows, the sample is allowed to cool while sputtered species are removed through diffusion to the source walls. As the applied power reaches its steady state condition, a sharp increase in signal can be seen for all background and contaminant gas species. This sharp increase, termed a prepeak, rapidly reaches a maximum within $0.5 \mathrm{~ms}$ of power initiation before decaying to a plateau value. The plateau region for both sputtered and nonsputtered species closely mimics steady state plasma conditions and behavior. Once power has been terminated, the signals for certain background species diminish to the baseline value. In contrast, the signals for sputtered species show no prepeak behavior, but show a large maximum, termed an afterpeak, after power termination. The explanation of prepeak and afterpeak behavior relates to changes in ionization that take place over the course of the pulse sequence.

When power is initiated, ionization is dominated by electron impact; electrons colliding with and ionizing gas phase species. Ionized sputtered atoms are not observed because the discharge gas ions must form first. Those ions, then, begin to sputter the sample cathode releasing the neutrals that are eventually ionized. Thus the ionized sputtered atom population always trails the discharge gas ion population. Only those atoms and molecules already in the gas phase exhibit prepeak behavior. As more discharge gas ions form and sputter the cathode, the sputtered atom and ionized sputtered atom population climbs and reaches a steady state values. Meanwhile, the prepeak declines over the next $2 \mathrm{~ms}$ to a steady state value. This decline corresponds with a decrease 
in the fraction of ionization arising from electron impact. Competing collisional ionization and recombination processes now become significant to the overall chemistry of the plasma.

From this point until the termination of applied power the glow discharge mimics steady state conditions. Both sputtered and nonsputtered species populations have reached a steady state, or plateau, value. Neutral sputtered species are ionized in the negative glow principally by electron impact and Penning ionization. Once power is terminated, all electron impact ionization ceases as the electrons thermalize. Upon thermalization, electrons recombine with discharge gas ions increasing the metastable population. It is significant that the formation of metastable atoms is not an instantaneous process. The time required for higher energy electrons to thermalize or "cool" explains why the afterpeak intensity maximizes $2-3 \mathrm{~ms}$ after pulse termination. With the absence of electron impact, and this enhanced population of metastable atoms, Penning ionization now becomes the sole ionization process. Using argon as a discharge gas, its metastable energies (11.5 and $11.72 \mathrm{eV}$ ) are too low to ionize neutral argon atoms, water or other contaminant gas species. Only gas phase atoms having ionization potentials below the metastable energy will exhibit afterpeaks. Atomic absorption studies have shown that the metastable argon atom population peaks within $2 \mathrm{~ms}$ of power termination, coinciding with the increase in ionized sputtered atom population. ${ }^{12}$

The analytical utility of modulated plasmas with time gated detection methods allows for the selection of regions within temporal profiles where 
analytical signals are at their maximum and background signals are at their minimum. ${ }^{13}$ Modulated or pulsed plasmas have been shown to have analytical utility when used with quadrupole, ${ }^{42}$ ion trap, ${ }^{17}$ and time of flight instruments. ${ }^{18,19}$ Recently, modulated plasma research has been extended to the application of micro second pulsed plasmas. ${ }^{14}$

As previously demonstrated, ${ }^{20}$ the glow discharge source operated in the pulsed, or modulated, power mode affords a number of distinct advantages over its steady state counterpart. Previous studies in this laboratory examined the differences in the temporal ion signals for analyte and discharge gas species. ${ }^{42}$ The presence of discrete time regimes affords the important advantage of temporal selectivity. ${ }^{16}$ This allows the observation of analyte ions during a time in which there is little or no interference from electron ionized species, affording an opportunity for the elimination of isobaric interferences attributed to the discharge support gas. Time-gated monitoring of the afterpeak region signal allows discrimination of discharge gas species formed by electron impact while enhancing the signal to noise of sputtered species ionized predominantly through Penning ionization. The separation of ${ }^{40} \mathrm{Ca}^{+}$and ${ }^{40} \mathrm{Ar}^{+}$is achieved through the use of a box car integrator coupled with a quadrupole mass spectrometer to yield time-gated mass spectrometric detection through.

\subsection{Experimental}

The glow discharge ion source and sample introduction system have been described in detail in a previous publication. ${ }^{4}$ The dc glow discharge was powered by a fast operational power supply (OPS-3500, Kepco, Flushing, NY). 
The power supply's output was driven and controlled by a pulse generator fabricated in house. The pulse generator allowed for control of the applied power level, frequency, and duty cycle. Radio frequency power was supplied by a 13.56MHz rf generator (Model RF 10-S, RF Plasma Products, Inc., Marlton, NJ) and coupled through an automatic matching network (Model AM-10, RF Plasma Products Inc., Marlton, NJ). The internal pulsing mode of the generator permitted control of the duty cycle, frequency and applied power. All data was collected for plasmas operating with duty cycles of $25 \%$ and frequencies of $50 \mathrm{~Hz}$. During all measurements a constant pressure of 0.55 torr argon was maintained. The dc glow discharge was operated under a pulsed current of $2.0 \mathrm{~mA}$ and applied voltage of $1.4 \mathrm{kV}$, whereas the rf glow discharge source was operated at a constant power of 130 watts.

All mass spectrometric measurements were made using a modified triplequadrupole mass spectrometer (ELQ-400, Extrel Pittsburgh, PA). The glow discharge ion source was positioned axially with respect to the ion entrance aperture of the mass spectrometer. Further modifications of the mass spectrometer include replacement of the $\mathrm{Cl} / \mathrm{El}$ ion source with a Bessel-box energy analyzer (Extrel Pittsburgh, PA), and installment of an Einzel lens assembly. Ions from both sources were sampled with the cathode positioned 14 $\mathrm{mm}$ from the ion exit orifice. The Einzel lens assembly focused ions from the ion exit orifice into the Bessel-box entrance aperture. In these experiments Besselbox served only to focus the ion beam because its energy-filtering cone was removed to allow for greater ion throughput. The triple quadrupole was operated 
with the first and second quadrupoles serving as rf-only ion guides with the third quadrupole serving as the mass analyzer.

Data collection was performed in three different modes. The first mode, used primarily for tuning and calibrating the mass-to-charge scale, gave the average signal over the entire pulse. This was accomplished by transferring the signal output from a continuous dynode amplifier (Galileo Electro-optic Corp., Strurbridge, MA) through a preamplifier (Combo-100, MIT, Boulder, CO) and into the computer data system (Teknivent Corp., St. Louis, MO), where the signal was averaged and integrated. In the second mode, ion temporal profile scans were digitally captured by monitoring the output of the continuous dynode with a 1-GHz oscilloscope (9370M, LeCroy, Chestnut Ridge, NY). Time-gated mass spectrometric detection, the third mode, was accomplished using a boxcar integrator to collect signal through a data gate. Signal from the electron multiplier was amplified using a preamplifier, split, and sent to two boxcar integrators. Both boxcar integrators were set to their lowest sensitivity value of $20 \mathrm{mV}$, and employed a fixed gate window of $0.15 \mathrm{~ms}$ with 30 pulse profiles being averaged for each data point collected. The data gate could be positioned through out the pulse cycle by the use of the digital delay generator. The output of the two averagers was processed by a computer using applications software (4162, EG\&G PAR, Princeton NJ). To further increase signal to noise, the resulting output from each boxcar integrator was summed into one resulting spectrum. The data were then transferred into a spreadsheet (Excel 97, Microsoft Corp., USA) and processed. 


\subsubsection{Sample Preparation}

Calcium doped iron samples were prepared using a standard Ca solution (Aldrich Chemical Co.) having an initial concentration of $995 \mu \mathrm{g} / \mathrm{mL}$. Using micropipettes and disposable tips (Eppendorf, Brinkmann, Instruments, Inc., NY) calculated of volumes of this solution were added to 1.000 gram of iron powder (Analytical Grade, J.T. Baker Chemical Co.) to yield reference powders of 0, 50, 100,150 , and $250 \mathrm{ppm}$. These powders were then dried over night in an oven at $95^{\circ} \mathrm{C}$. After drying each sample was homogenized for 2 minutes using a laboratory mixer (Wig-L-Bug, SPEX, Edison, NJ). An aliquot of each standard was then pressed into a sample cathode disks at 4500 psi for 2 minutes. The resultant sample disks had a diameter of $0.200 "$. The sample was then mounted on the direct insertion probe and inserted into the mass spectrometer to pump for no less than two hours. The sample was then preburned for 90 minutes before mass spectrometric measurements were taken. 


\subsection{Results and Discussion}

GDMS systems based on quadrupole mass analyzers allow for rapid mass scanning with detection limits at sub-ppm levels, and are used for the characterization and production control of high purity materials. Limitations to the use of such instruments arise from isobaric interferences produced in the glow discharge source coupled with the low resolving ability of quadrupole mass spectrometer. Because quadrupoles are limited to near unit resolution many isobaric interferences cannot be resolved, thus, hampering the detection of analytes having the same nominal mass-to-charge ratio as the interference. ${ }^{43}$ This problem is clearly illustrated for the determination of calcium because major ions for both the argon and calcium appear at $\mathrm{m} / \mathrm{z} 40$.

Pure iron samples were monitored throughout the pulse cycle for both dc and rf powered glow discharge sources. During the plateau region, ions are formed by a combination of electron impact and Penning ionization processes. Monitoring of this temporal regime was accomplished by positioning the delay gate $4.8 \mathrm{~ms}$ with respect to the onset of the applied power square wave. Figure 5.3.1.1A illustrates the $50 \mathrm{~Hz}, 25 \%$ duty cycle, temporal response for a major ionized sputtered species $\left({ }^{56} \mathrm{Fe}+\right)$, a major ionized discharge gas species $\left({ }^{40} \mathrm{Ar}^{+}\right)$ and the delay gate window. DC spectra collected show an argon ion contribution at $\mathrm{m} / \mathrm{z}$ of 40 (Figure 5.3.1.1B). This is a result of electron impact ionization taking place while the applied power pulse is in the on mode. Notice the intensity of argon ion isotope signal when compared to the major iron isotope signal. The support gas argon having a pressure of 0.55 torr is the dominant gas species, however intensity of the ${ }^{56} \mathrm{Fe}^{+}$isotope is over eight times as abundant. Results 
such as this support the assignment of Penning ionization as the dominant ionization mechanism in steady state discharges. Other major contributors to the spectrum are argon hydride, ${ }^{40} \mathrm{Ar}^{1} \mathrm{H}^{+}$, the various minor iron isotopes, ${ }^{54} \mathrm{Fe}^{+}$, ${ }^{57} \mathrm{Fe}^{+}$, and ${ }^{58} \mathrm{Fe}^{+}$, and the chromium isotope, ${ }^{52} \mathrm{Cr}^{+}$. The chromium signal arises from sputtering of the stainless steel sample disk holder, whereas argon hydride arises from associative ionization involving argon metastables and hydrogen.

The data gate was then positioned to $6.6 \mathrm{~ms}$ after the onset of the applied power pulse for ion sampling of the afterpeak, Figure 5.3.1.2A. As a result, the acquired dc spectrum shows an improvement of seven-fold in ionized sputtered atom intensity, Figure 5.3.1.2B. During the afterpeak sputtered atoms ionize predominantly by a Penning mechanism, metastables colliding with neutral sputtered atoms. The increase in ionized sputtered atom intensity is attributed to the large population of metastables present once power is terminated. Once power is terminated, signal for electron ionized species declines and argon-ion electron recombination dominates. Complete dissipation of the argon ion population occurs rapidly under these conditions.

Further investigations using the boxcar integrators were preformed in order to identify temporal regions that were free from any argon ion contribution. The data gate was systematically moved across the afterpeak time regime in 0.1 $\mathrm{ms}$ intervals starting at $6.6 \mathrm{~ms}$ for both rf and dc discharges. The probe position, discharge gas pressure and discharge current and power were kept at their previous values. Each boxcar integrator was operated at its highest sensitivity 

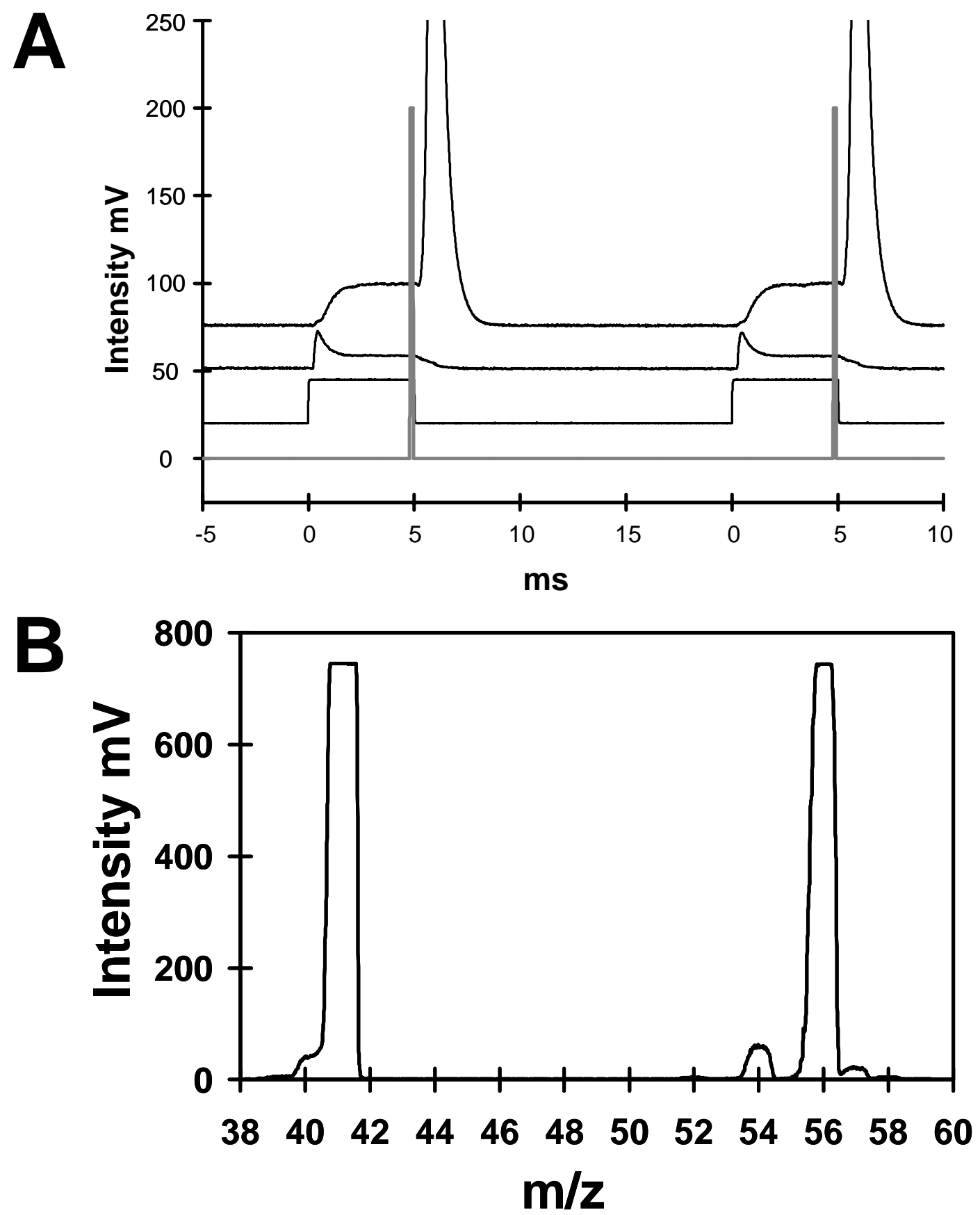

Figure 5.2.1.1 A) Temporal response of (a) the analyte $\left({ }^{56} \mathrm{Fe}^{+}\right)$, (b) discharge gas $\left({ }^{40} \mathrm{Ar}^{+}\right)$, (c) the applied power pulse and (d) the data gate positioned at $4.8 \mathrm{~ms}$. B) Mass spectrum acquired using the modulated dc power supply and a delay gate of $4.8 \mathrm{~ms}$. 

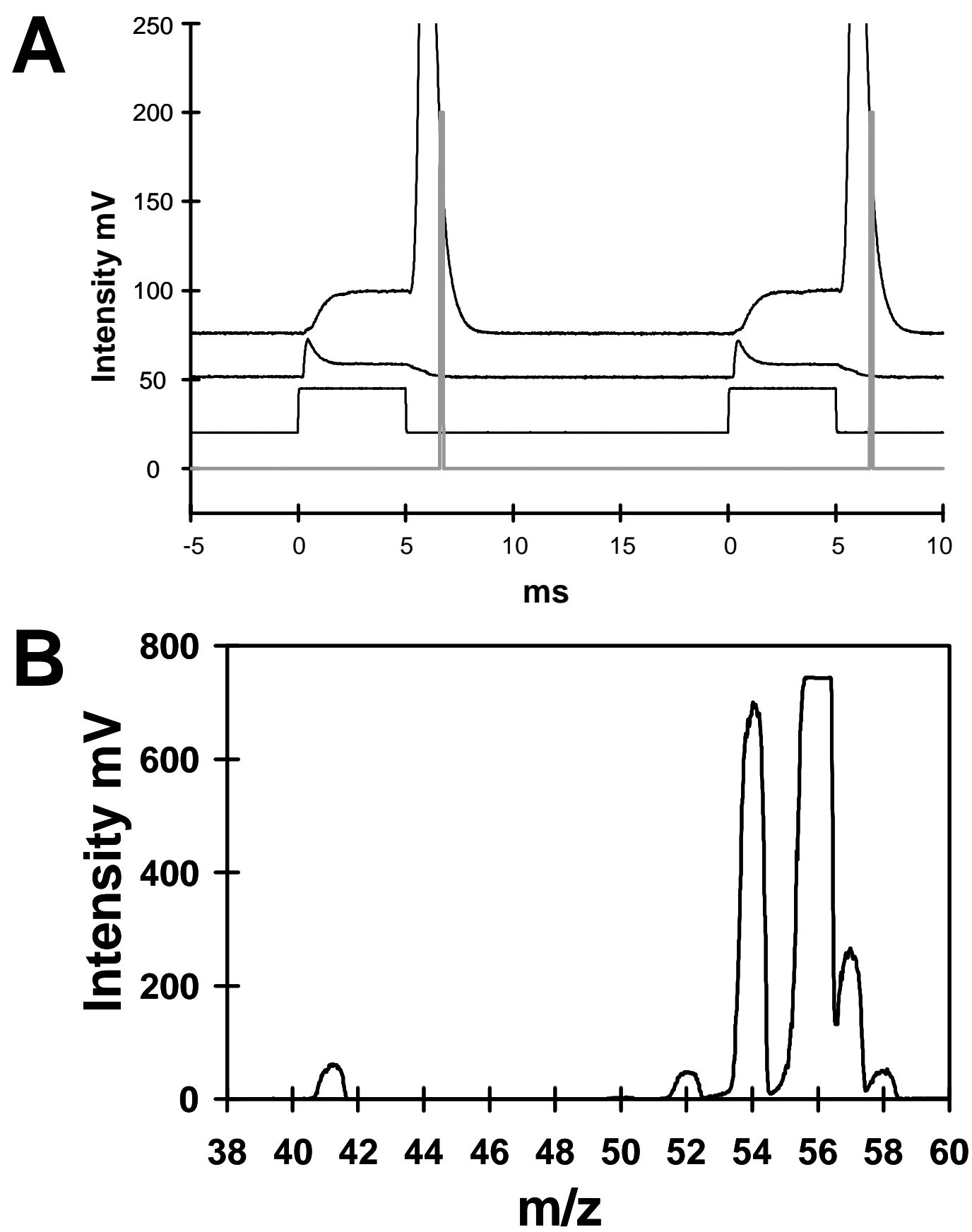

Figure 5.2.1.2 A) Temporal response of (a) the analyte $\left({ }^{56} \mathrm{Fe}^{+}\right)$, (b) discharge gas $\left({ }^{40} \mathrm{Ar}^{+}\right)$, (c) the applied power pulse and (d) the data gate positioned at $6.6 \mathrm{~ms}$. B) Mass spectrum acquired using the modulated dc power supply and a delay gate of $6.6 \mathrm{~ms}$. 
setting of $20 \mathrm{mV}$. The mass rage of interest centers at $\mathrm{m} / \mathrm{z}$ of 40 and the spectra are recorded for the range from $38 \mathrm{~m} / \mathrm{z}$ to $43 \mathrm{~m} / \mathrm{z}$.

As explained earlier the data coming from the mass spectrometer's continuous dynode electron multiplier is amplified via a preamplifier and then split between two boxcar averagers. The averaged signal was then processed and the output of each boxcar was added together by the boxcar software. Five such coadded scans of the same delay were averaged using a spreadsheet. The resultant averaged coadded scan was plotted and then examined for any signal contribution from the argon ion at $\mathrm{m} / \mathrm{z}$ of 40 . As the delay gate is swept further out into the afterpeak, signal for the argon isotope subsides reaching the baseline at $7.0 \mathrm{~ms}$.

These experiments were repeated for the rf powered glow discharge. Increasing the position of the delay further out in the afterpeak allows the removal of argon ion by diffusion and recombination processes. Using the most sensitive setting for the boxcar, a delay positioned at $6.8 \mathrm{~ms}$ was found to be free from any argon ion contribution. The data support the premise that ionization and recombination processes happen on a faster time scale in a rf powered glow discharge than in its dc counterpart. This temporal difference in afterpeak maximum between these two plasmas will be the subject of future investigations in this laboratory.

Calcium doped iron standards were examined using plasma operating conditions, preburn times and data acquisition parameters identical to those used for the pure iron standards. Time gated mass spectrometeric detection was 
accomplished by positioning the delay gate at $7.0 \mathrm{~ms}$ and $6.8 \mathrm{~ms}$ for $\mathrm{dc}$ and $\mathrm{rf}$ powered glow discharge sources respectively. Figures 5.3.1.4.A and $B$ are spectra acquired for pure iron and calcium samples of 50, 150, 250 ppm using the appropriate delay for both dc powered and rf powered plasmas.
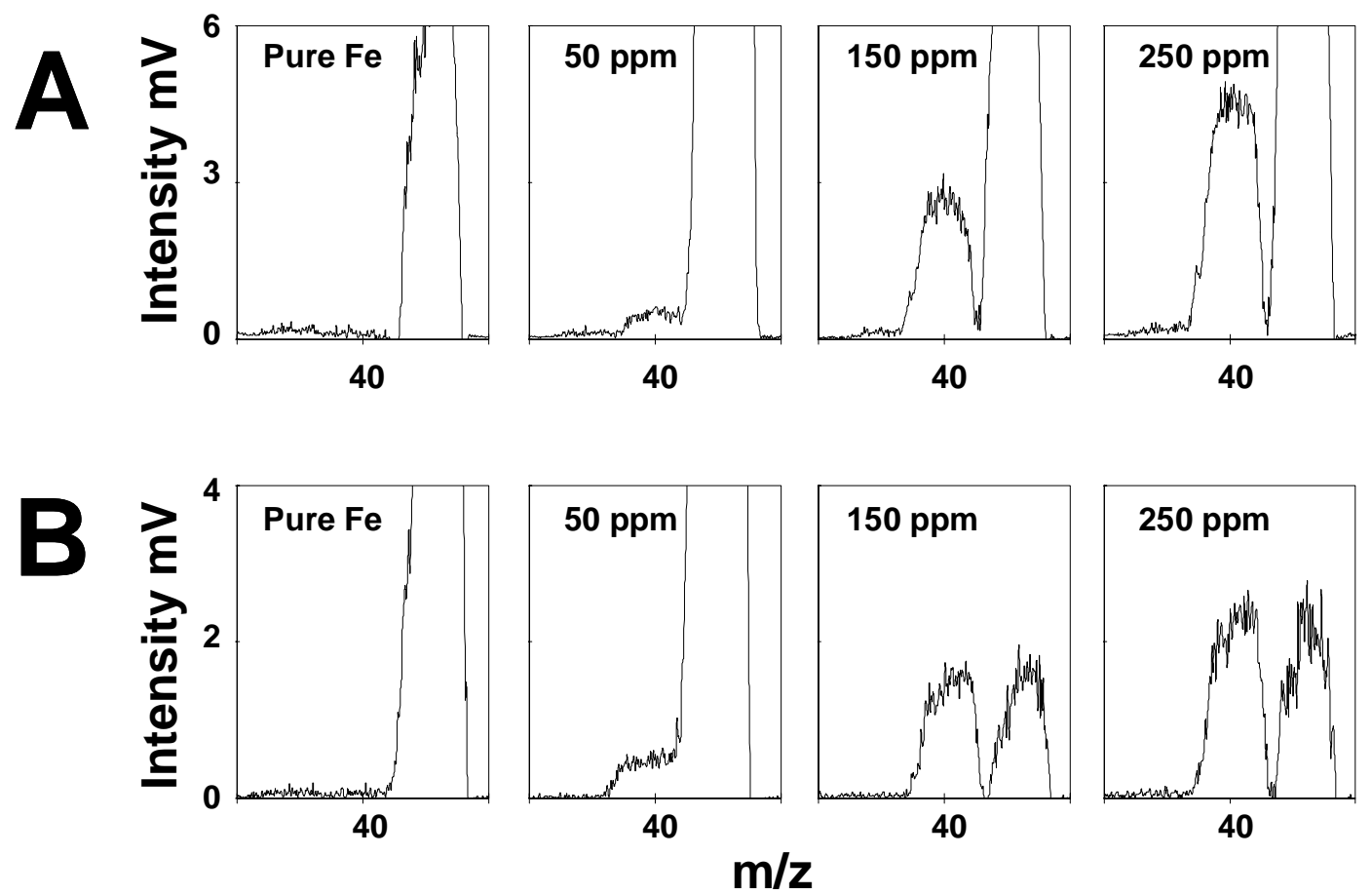

Figure 5.3.1.3Average spectrum of five coadded scans obtained for $0,50,100,150$, and $250 \mathrm{ppm} \mathrm{Ca}$ in pure Fe samples with the modulated (A) dc glow discharge using a data gate delay of $7.0 \mathrm{~ms}$, and (B) the rf glow discharge source using a delay gate positioned at $6.8 \mathrm{~ms}$. 
Calibration curves for both dc and rf powered glow discharge sources were constructed with the data collected from the five calcium standards, Figure 5.3.3.4A (dc) and B (rf). The plotted points in both are the average of the five replicate scans bracketed with $3 \sigma$ error bars. The dashed lines represent the linear regression of the data collected. The regression data was calculated using a spreadsheet and the correlation coefficients for the dc and rf curves were 0.9995 and 0.9915 respectively. Detection limits for both were extrapolated to be $23.6 \mathrm{ppm}$ for the dc and $6 \mathrm{ppm}$ for rf. The $\mathrm{y}$-intercept of both rf and dc calibration curves were defined at or below zero when factoring in the calculated standard deviation of regression line. Deviations in linearity arise from irreproducibility in sample preparation (weighing, drying, mixing and pressing steps) and discharge parameters (pressure, current, and sample position). 
A

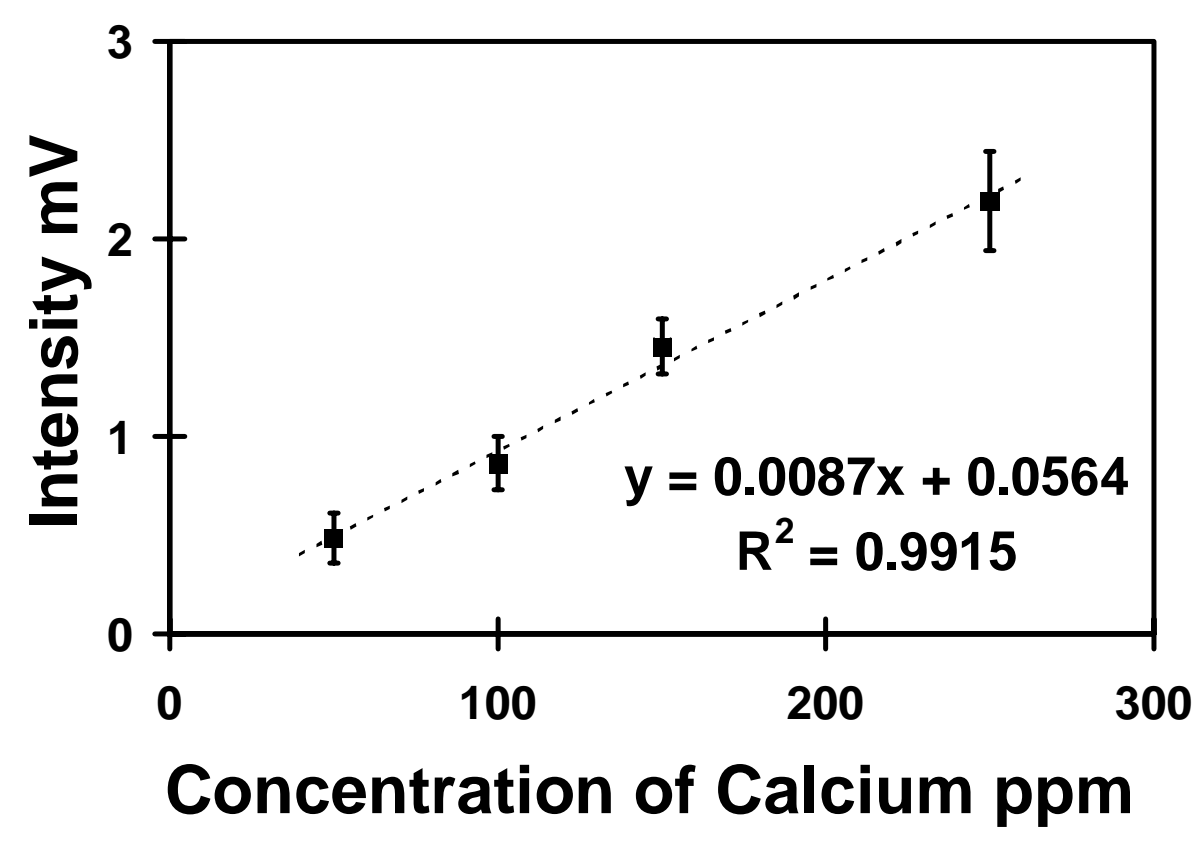

B

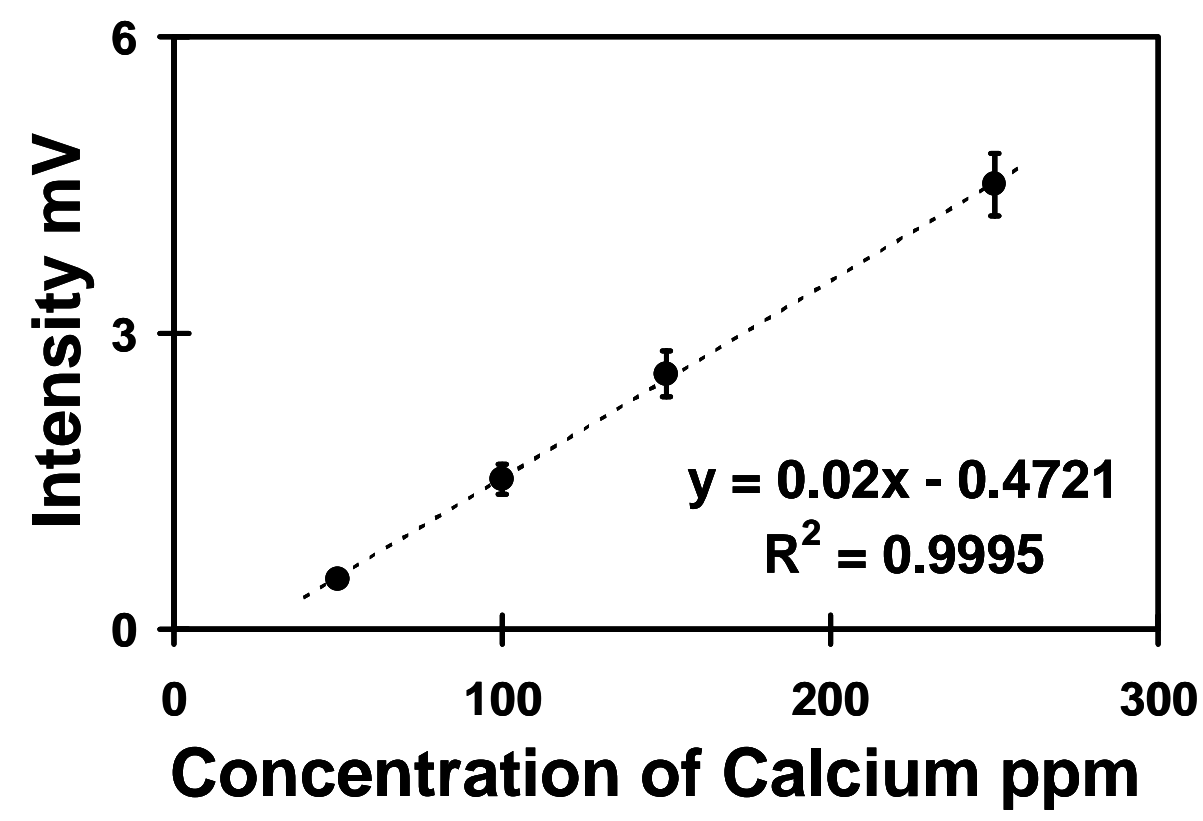

Figure 5.3.1.4 Calibration curves for calcium standards using a (A) dc powered discharge and a gate delay at $7.0 \mathrm{~ms}$, and (B) a rf powered discharge and a gated delay of $6.8 \mathrm{~ms}$. 


\subsection{Conclusions}

Modulated glow discharge sources exhibit signal enhancement for sputtered species while affording the added advantage of temporal discrimination against interferences. The calibration curves constructed for both dc and rf plasmas demonstrate complete discrimination against electron ionized species in both dc and rf glow discharges by time gated mass spectrometeric detection. Because argon was the most abundant gas species present in the ion source, other lower abundance isobaric interferences produced by electron impact should provide similar if not better results. Temporal profiles produced by modulated glow discharges are dependent upon discharge parameters such as pressure, spatial region, current and voltage. Future studies will focus on the various ion kinetic energies found in low pressure modulated discharges (0.4 -1.0 torr). 


\section{Detection of Volatile Organic Compounds Using the Pulsed Glow Discharge lon Source Coupled with a Time-of-Flight Mass Analyzer}

\subsection{Introduction}

Many volatile organic compound (VOCs) speciation methods are hybrid methods involving a chromatographic separation followed by mass spectrometric detection. In such schemes, identification is inferred from the separation method while relative concentrations are determined mass spectrometerically. These methods are employed in modern laboratories in the form of gas and liquid chromatography mass spectrometric systems. Chromatography methods separate and concentrate different chemical species based on phase partitioning. Once separated, the sample is analyzed mass spectrometrically, where further information and identification of the analyte may be deduced based on its inherent mass-to-charge ratio. However, following chemical separation and previous to any mass spectrometric detection, the analyte most be ionized. To date there are numerous possible methods for ionization, however, they can be classified into hard and soft ion sources.

The common ionization method is electron ionization (EI) in which the compound is atomized or fragmented. This method readily provides both elemental and structural information, because compounds of the same class will have similar elemental compositions and fragmentation patterns. In contrast, soft ionization, such as chemical ionization $(\mathrm{Cl})$, yields molecular weight information because the unfragmented molecule is ionized and detected intact. It is very easy to see that these two methods are complements of each other. To date, no 
ion source has been developed that grants both $\mathrm{El}$ and $\mathrm{Cl}$ characteristics simultaneously and independently of one another.

In previous investigations the pulsed glow discharge source has demonstrated an ability to permit the separation of electron-ionized species from Penning ionized species. Penning ionization is a relative soft ionization process, where analyte molecules are ionized by potential energy transfer in collisions with metastable argon atoms. This chapter describes initial investigations into the determination of volatile organic compounds by pulsed glow discharge timegated-mass spectrometry.

\subsection{Experimental}

The glow discharge cell is constructed of 1 inch tubing fixed to a NPT tapped double sided 2.75 inch conflat flange (MDC, Hayward, CA, USA). A gas inlet port and the chamber pressure monitor (Hasting Teledyne-Radist, Hampton, VA, USA) are fixed to the NPT tapped ports. A $1 / 2$ inch probe inlet is connected to this chamber, and a $1 \mathrm{~mm}$ ion exit orifice permits the extraction of ions into the time-of-flight mass analyzer. The glow discharge probe was described in a previous chapter, Chapter 2.1. The glow discharge cathode is a $5 \mathrm{~mm}$ diameter disk of tantalum. Tantalum was chosen for because of its low sputtering characteristics. Sample introduction was accomplished through a leak valve plumbed into the direct insertion probe inlet. VOC samples were contained in a sample vial attached the leak valve (22 Series, Whitney, Co., Highland Heights, $\mathrm{OH}, \mathrm{USA}$ ) via a $1 / 4$ Cajon ultra-torr fitting (Swagelok, Macedonia, OH, USA). 
Pulsed operation of the glow discharge source was accomplished by using a dc power supply (Model OPS-3500, Kepco, Inc., Flushing, NY, USA) controlled by a digital delay generator (Model DG 535, Stanford Research Systems, Sunnyvale, CA, USA). The digital delay generator allows adjustment of the pulse width, duty cycle and gate delay. This gate delay triggers the application of the ToF repeller plate, which will inject a sample packet of ions orthogonally into the flight tube for analysis. Adjustment of this delay allow for time-gated detection of different ion processes occurring throughout the pulse cycle.

The time-of-flight mass spectrometer is an orthogonal sampling instrument (Model D-850, R.M. Jordan, Grass Valley, CA, USA) operated in the reflectron mode. Ion injection was preformed orthogonal to the glow discharge source, allowing for pulsed injection of sample ions throughout the pulse cycle. The system's operating conditions are presented in Table 6-1. The signal output from the $40 \mathrm{~mm}$ dual microchannel plate detector (Galileo Electro-optic Sturbridge, MA, USA) was monitored and collected using a $500 \mathrm{MHz}$ digital storage oscilloscope (Model TDS 520, Tektronix). The data was imported into a spreadsheet (Microsoft Excel 97, Microsoft, Redmond, WA, USA) for analysis. 


\begin{tabular}{|c|c|}
\hline \multicolumn{2}{|l|}{ Pressure } \\
\hline Ionization source & 0.3-0.6 torr \\
\hline Mass Analyzer & $5.0 \times 10^{-6}$ torr \\
\hline Applied GD Potential & $1.0 \mathrm{kV}$ \\
\hline Pulse length & $5 \mathrm{~ms}$ \\
\hline Duty cycle & $25 \%$ \\
\hline Sampling orifice diameter & $1.0 \mathrm{~mm}$ \\
\hline Skimmer orifice diameter & $1.0 \mathrm{~mm}$ \\
\hline Repeller pulse time & $2.5 \mu \mathrm{s}$ \\
\hline Flight path & $2 \mathrm{~m}$ \\
\hline \multicolumn{2}{|c|}{ Time of Flight Potentials } \\
\hline Skimmer & $-2.0 \mathrm{kV}$ \\
\hline Repeller & $+400 \mathrm{~V}$ \\
\hline Accelerator & $-2.0 \mathrm{kV}$ \\
\hline Extractor & Ground \\
\hline Flight Tube & $-2.0 \mathrm{kV}$ \\
\hline Deflector XY1 & $-2.4 \mathrm{kV}$ \\
\hline Deflector XY2 & $-2.0 \mathrm{kV}$ \\
\hline Detector & $-2.0 \mathrm{kV}$ \\
\hline
\end{tabular}

Table 6-1 


\subsection{Results and Discussion}

The glow discharge power pulse profile and its temporal ionization characteristics are illustrated in Figure 6.3.1.1. During the first few hundred microseconds of the pulse cycle, ionization processes are dominated by electron ionization and mimic electron impact (EI) sources. As mentioned in previous chapters, this temporal regime has been termed the prepeak, based on mass spectrometeric observations of the argon ion population. This sharp rise and fall of the argon ion population arises from the initial high flux in electron density, followed by equilibrium of competing ionization and recombination processes.

\section{Prepeak}

\section{Electron Ionization}

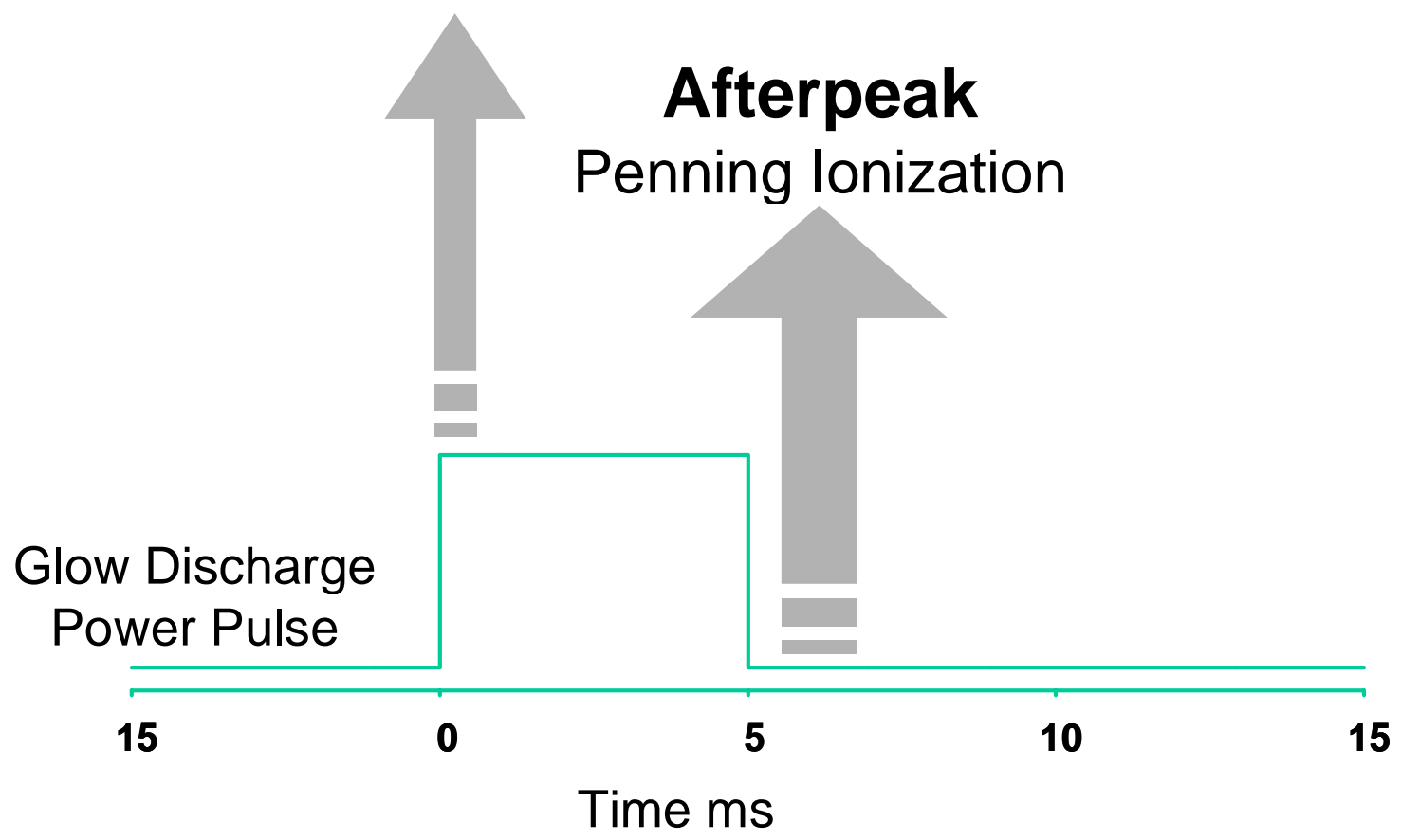

Figure 6.3.1.1 Illustration of the glow discharge pulse sequence and the temporal regions of interest. 
However, the prepeak phenomenon is not limited to argon species, but any species in the gas phase prior to power initiation. This can be readily seen in Figure 6.3.1.2 a taken $0.30 \mathrm{~ms}$ into the pulse cycle. The most dominant species present are attributed to the discharge support gas of argon, ${ }^{40} \mathrm{Ar}^{+}$and ${ }^{41} \mathrm{ArH}^{+}$, yet contaminant gas species such as ${ }^{19} \mathrm{H}_{3} \mathrm{O}^{+}$and ${ }^{28} \mathrm{~N}_{2}{ }^{+}$are visible. Species from the sacrificial cathode, ${ }^{181} \mathrm{Ta}^{+}$, are not detected. This is simply because tantalum was not present in the gas phase prior to power initiation. Figure 6.3.2.1 b was taken under similar conditions with the addition of a small amount of p-xylene. This was accomplished by leaking a small amount of the volatile organic into the discharge chamber. This spectrum differs considerably from the previous, as the fragment ions of $p$-xylene are now incorporated in it. This fragmentation pattern was compared to the pattern observed in a NIST electron ionization spectra recorded at $70 \mathrm{eV}$, Figure 6.3.1.3 $\mathrm{a}$ and $\mathrm{b}$. The similarities between these two patterns suggest that the current glow discharge operating conditions found during the prepeak are comparable to standard electron ionization sources currently employed in most laboratories.

Upon power termination, ionization is dominated by Penning ionization, as argon ions and thermal electrons recombine to form argon atom metastables. As previously discussed, Penning ionization has been attributed to be the major source of sputtered atom ionization. This process continues for several milliseconds after power termination. However, in the absence of the electric field placed upon the cathode surface, electron ionization halts. 


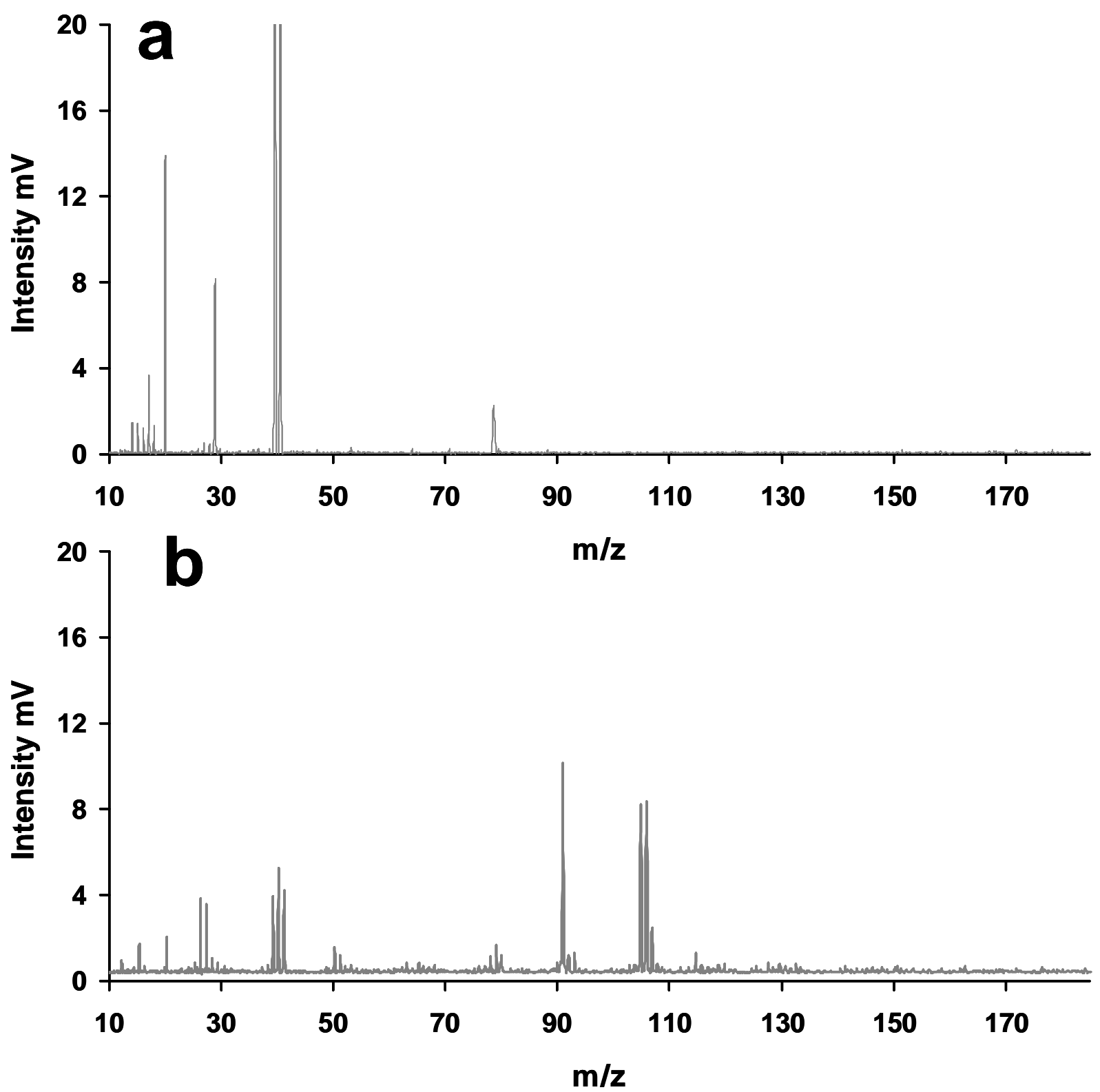

Figure 6.3.1.2 Spectra acquired during the prepeak regime $0.3 \mathrm{~ms}$ during the glow discharge pulse cycle (a) without and (b) with the addition of p-xylene. 

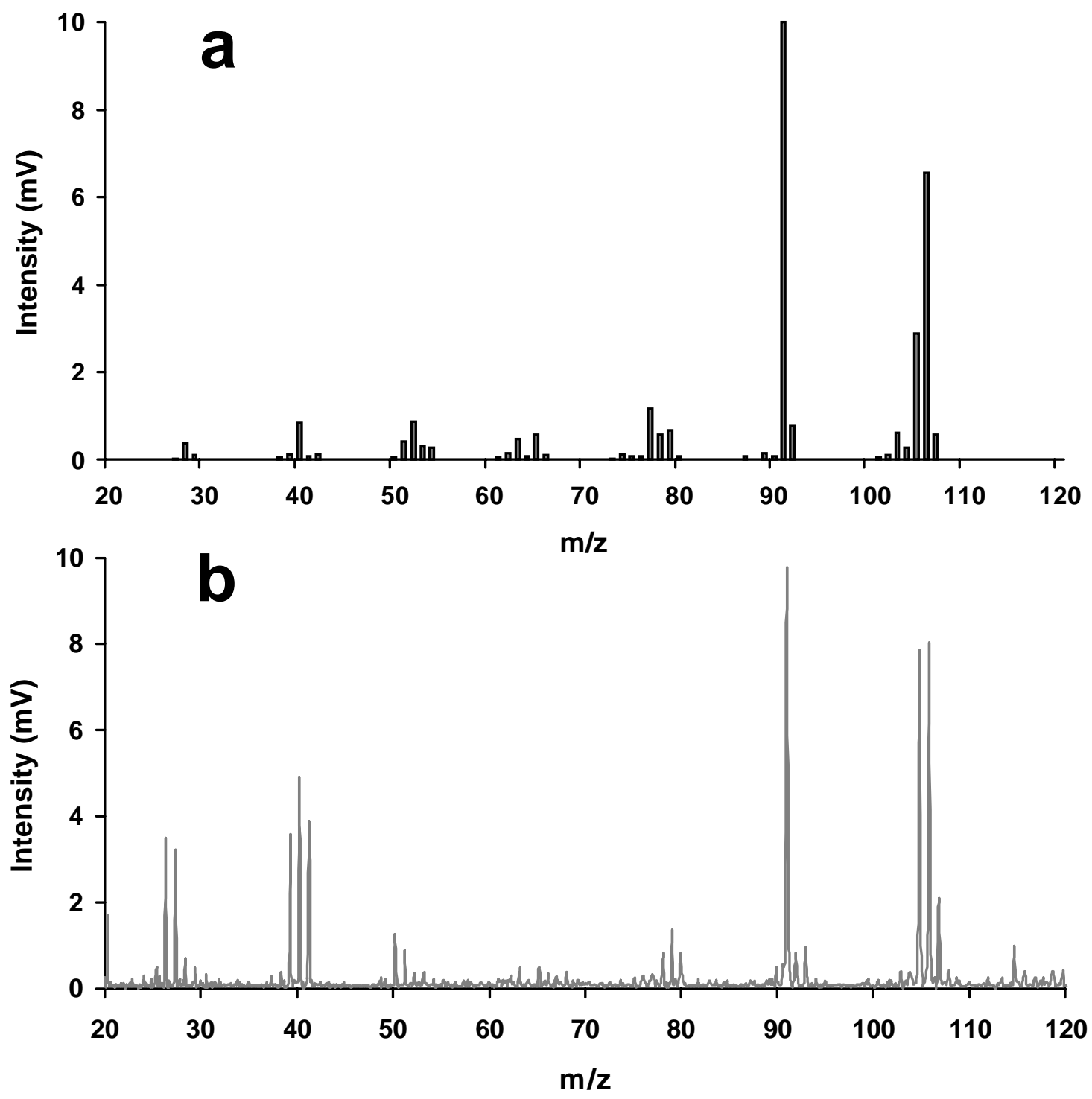

Figure 6.3.1.3 Comparison (a) electron ionization spectra of a NIST $70 \mathrm{eV}$ of $\mathrm{p}$-xylene with (b) a spectrum acquired during the prepeak time regime $0.3 \mathrm{~ms}$ into the pulse cycle. 
This effect is illustrated in spectrum taken during the afterpeak, $6.5 \mathrm{~ms}$ during the pulse cycle, Figure 6.4.1.4 a. The only species detected is the tantalum ion having a $\mathrm{m} / \mathrm{z}$ ratio of 181 , while no argon or residual gas species are present. This is due to the absence of electron ionization and that the ionization potentials of these species are beyond the energy associated with the argon atom metastable (11.5 and $11.72 \mathrm{eV})$. The judicial choice of tantalum as the sacrifice cathode was based on its low sputtering yield and because it is nearly monoisotopic. Spectra taken with the addition of $p$-xylene under the similar conditions demonstrate the presence of both $\mathrm{Ta}^{+}$and $\mathrm{p}$-xylene ions, Figure 6.3.1.4 b. The $\mathrm{m} / \mathrm{z}$ ratio of ${ }^{180} \mathrm{Ta}^{+}$is once again attributed to the cathode material; a loss in its signal intensity suggests that metastable population has been quenched. The most possible candidate for a quenching agent can be deduced to be the $\mathrm{m} / \mathrm{z}$ ratio found at 106 . This mass-to-charge ratio matches that for an intact $p$-xylene ion. The absence of fragment ions further suggests that the mechanism of ionization is soft and analogous to that found in chemical ionization sources. 

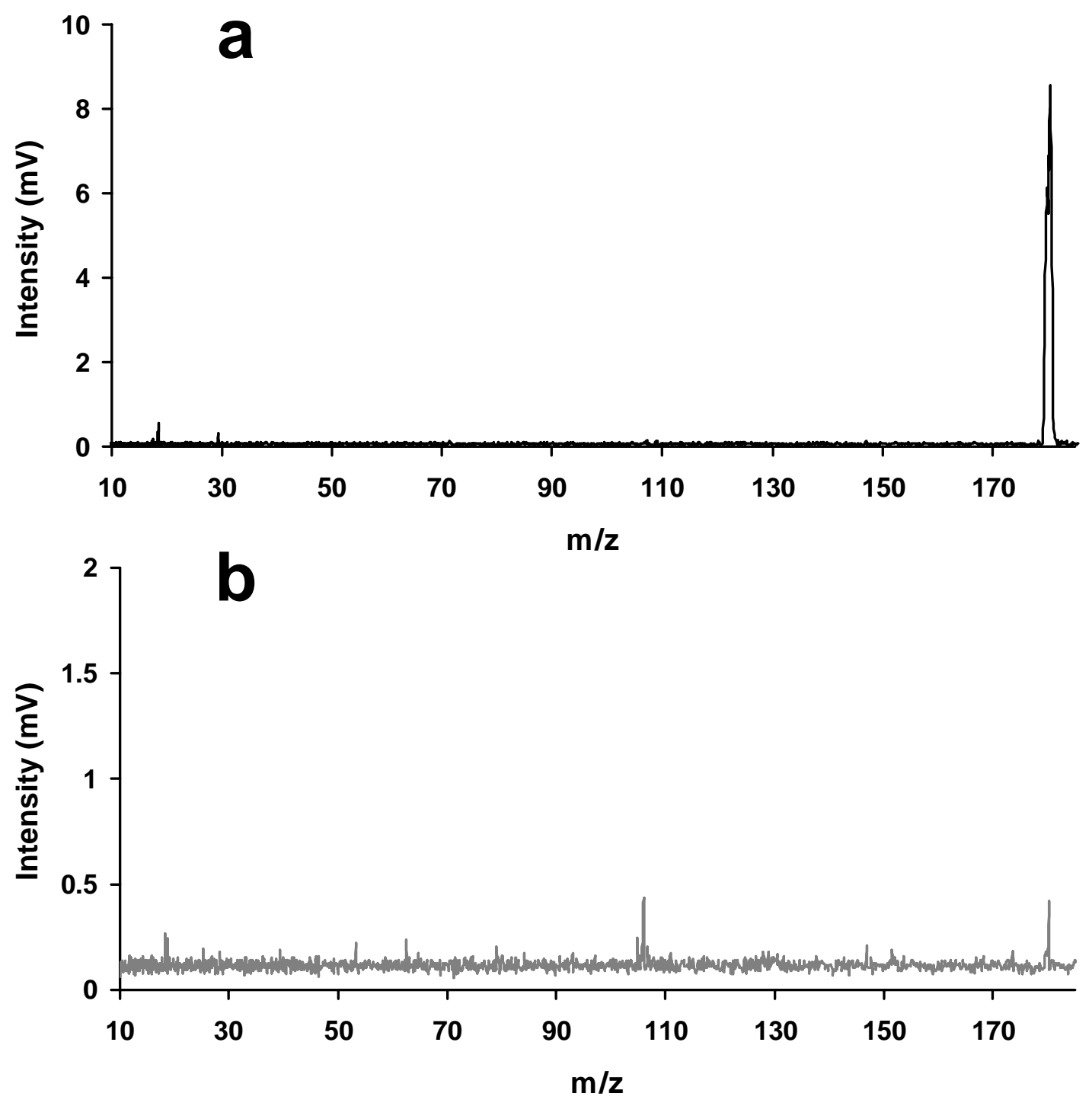

Figure 6.3.1.4 Spectra acquired during the afterpeak regime $6.5 \mathrm{~ms}$ during the glow discharge pulse cycle a) without and b) with the addition of p-xylene. 


\subsection{Conclusions}

The use of a millisecond glow discharge source to provide both structural and molecular information has been demonstrated. The dynamic nature of the pulsed glow discharge source allows for analysis of a compound subjected to electron and chemical ionization like processes. The electron ionization spectra collected over the prepeak regime mimic electron impact spectra taken at $70 \mathrm{eV}$. Ion collection during the afterpeak allows for the detection of the intact molecular ion without fragmentation. These features make this technique amenable to chemical detection of gas phase molecular species, in which the identification is deduced by structural and molecular weight information. Future investigations will apply this method for the speciation of solids, liquids, and as a detector for gas chromatography. 


\section{Characterization of a Pulsed Glow Discharge Laser Ablation System Using Optical Emission}

\subsection{Introduction}

Laser-based sampling methods permit direct solid sampling from both conductive and nonconductive materials with minimal sample preparation. Added advantages include the ability for micro-probing and depth-profiling. Elemental laser-based sampling encompasses a variety of hyphenated techniques employing both optical and mass spectrometric detection. These techniques can enhance analytical performance by increasing ionization and excitation of the ablated atom population. The most prevalent hybrid technique involves the inductively coupled plasma (ICP) source. ${ }^{44,45}$ Only a few publications exist that investigate the utility of using a secondary glow discharge cathode as an alternative to the ICP source. ${ }^{46,47,48}$

Laser sampling coupled to a glow discharge plasma ion source may afford a number of advantages over existing techniques. Optical and mass spectrometric detection following direct sample introduction into a glow discharge plasma may reduce fractionation problems associated with laser-based ICPMS. ${ }^{49,50}$ Laser-based techniques simplify sampling of nonconductors over standalone glow discharge methods. The analysis of nonconductors using dc glow discharge plasmas requires the fabrication of a pressed pellet consisting of powdered sample mixed with a metallic binder. ${ }^{51}$ Alternatively, the application of rf potentials or secondary (sacrificial) cathodes may eliminate the real for a conducting matrix, but both still require the sample to be machined into a suitable 
cathode geometry. ${ }^{52}$ In both cases additional sample preparation time is required prior to analysis.

Previous investigations involving laser sampling coupled to glow discharge sources have included both optical and mass spectrometric detection. lida used a hollow cathode and ablated sample from an adjacent cell. ${ }^{46}$ Vaporized material was then transferred via a tube into the hollow cathode cell, where excitation occurred. Emission was detected using a spectrometer with a photodiode array or PMT, and demonstrated analytical sensitivities at the $\mu \mathrm{g} / \mathrm{g}$ level. Hess used quadrupole mass spectrometric detection in the analysis of both conductors and nonconductors. ${ }^{47}$ Here, the laser target and secondary cathode were contained in the same chamber. Ionization of ablated material was accomplished by a steady state dc-glow discharge. Hess examined parameters (including pressure and distance) that affect the transport of ablated material into the negative glow. His experiments also revealed the negative impact of sputtered material redeposited on the laser target's surface.

Since its introduction by Harrison, there has been an increasing interest in the glow discharge ion source operated in the pulsed power mode..$^{20,12,21}$ When compared to their steady state counterparts, pulsed glow discharge ion sources enhance atomization and excitation/ionization processes through the application of higher applied potentials. An added advantage of the pulsed glow discharge source is the presence of discrete time regimes during which sputtered analyte species are at a maximum and background gas species are at a minimum. These regimes are termed "prepeak", during which electron ionization is 
dominant; and "afterpeak", during which Penning ionization is dominant.

Previous pulsed plasma research has demonstrated complete temporal discrimination of electron ionization and Penning ionization using time-gated techniques. ${ }^{18,22}$ Current research has demonstrated that, by accessing these different temporal regions, concurrent molecular and structural information can be obtained from vapor introduced into a pulsed glow discharge ion source..$^{36,23}$ Appropriate timing of a laser sampling pulse to access each of these temporal regions will allow for solid molecular analysis, as well as elemental analysis.

A typical pulsed glow discharge sequence consists of the applied power square wave, varying from $2-5 \mathrm{~ms}$ in length, followed by power termination. At power onset, electrons are accelerated away from the glow discharge cathode surface and attain energies sufficient to ionize the argon discharge gas, which has an ionization potential of $15.76 \mathrm{eV}$. Because this electrical breakdown condition is similar to ionization of an electron impact source, time-gated detection over the first hundred microseconds will yield fragmentation information for molecular species. Upon reaching the negative glow region of the plasma, collisional energy loss results in an average electron energy of $2 \mathrm{eV} .{ }^{28}$ Cooled electrons are now able to recombine with argon ions to form metastable argon atoms. When the applied power is terminated, competing processes are reduced and a surge in metastable Ar atoms is produced. These metastable species can only release energy and return to the ground state through processes, such as collisions with other gas species. During a collision the metastable atom's energy is released and may lead to excitation/ionization of the collision partner. 
The ionization process is known as Penning ionization. The ${ }^{3} \mathrm{P}_{2}$ and ${ }^{3} \mathrm{P}_{0}$ metastable states of Ar occur at energies of $11.52 \mathrm{eV}$ and $11.72 \mathrm{eV}$, and are more than sufficient to ionize most neutral elemental species. For molecular analysis, time-gated detection over this Penning ionization region will yield information on intact molecular ions.

The key to exploiting the potential advantages for solid sampling is optimizing the spatial overlap of ablated material with the ionizing medium during the temporal region with the desired ionization characteristics. We have investigated parameters (including pressure, and sample and cathode distances) that affect both transport and ionization/excitation of laser ablated copper in the pulsed glow discharge plasma. Special emphasis is placed on maximizing the detection of copper species excited by metastable atoms found during the afterpeak time regime. This involves both transport of ablated material and diffusion of the metastable populations.

\subsection{Experimental}

A schematic of the experimental setup is shown in Figure 7.2.1.1. The glow discharge source consists of a discharge chamber and two direct insertion probes for laser sample and cathode introduction. The discharge chamber is a six-way, high vacuum, stainless steel cross (MDC, Hayward, CA). Side ports on the cross consist of a laser ablation probe inlet with the remaining ports fitted with stainless steel suprasil optical view ports (Heraeus Quartz, Duluth, GA). The glow discharge probe inlet was positioned orthogonally to the laser target inlet and monochromator slit entrance. Both probe inlets consist of a ball valve fitted 
with a $1 / 2$ " adapter that allowed direct probe insertion without breaking the system vacuum. Pressure in the discharge chamber was monitored using a pirani pressure gauge (Edwards PRL-10).

The glow discharge direct insertion probe has been described in a previous paper. ${ }^{53}$ The glow discharge cathode is a tantalum disk, chosen for its low sputtering characteristics. Timing of the glow discharge pulse is achieved as follows: A synchronous TTL pulse from the laser Q-switch triggers a delay generator (DG535, Stanford Research Systems, Inc. CA) used to define the discharge pulse duration and delay with respect to the laser pulse. Output from the delay triggers the glow discharge high voltage power supply (OPS 3500, Kepco Inc, NY). The delay on the power pulse is set to sweep over the next laser pulse. A 2ms glow discharge pulse, operated at $10 \mathrm{~Hz}$ and $1 \mathrm{kV}$ (unless stated otherwise), was used. A pulsed Nd:YAG laser was used for sample ablation, with a $10 \mathrm{~Hz}$ repetition rate, and a $10 \mathrm{~ns}$ pulse width. The $1064 \mathrm{~nm}$ beam was doubled to $532 \mathrm{~nm}$ using a KDP second harmonic generator, followed by beam separation with a pellinbroca prism. Using $3 \mathrm{~mJ}$ per pulse, the $532 \mathrm{~nm}$ beam was focused onto the copper target with a $200 \mathrm{~mm}$ focal length plano convex lens. Ablation is performed at the focal point, with an estimated power density of $1 * 10^{9} \mathrm{~W} / \mathrm{cm}^{2}$. Data was obtained from an average of 128 laser shots, with the sample being rotated between each acquisition. The laser targets were fabricated from a copper rod (SRM 855, NIST, Gaithersburg, MD) into disks having a 0.240 " OD, which were attached to the $1 / 2$ " laser target direct insertion probe. 
Optical detection was performed orthogonally to the laser target by imaging the center of the glow discharge chamber onto the entrance slit of an f/4.1 monochromator (Triax 320, ISA, Inc, NJ) with a $128 \mathrm{~mm}$ f.I. biconvex lens. Entrance and exit slits were set at $300 \mathrm{um}$, resulting in a bandpass of $0.7 \mathrm{~nm}$. Output from the photomultiplier tube (Hamamatsu R955) was monitored with a digital storage oscilloscope (TDS 220,Tektronix Inc, USA) through a $50 \mathrm{ohm}$ connector. Synchronization of the discharge and laser pulses was also monitored on the oscilloscope. Emission temporal profiles were captured using the oscilloscope and then transferred to a spreadsheet for data analysis. In each investigation, the emission from the copper atom line ( $\mathrm{Cu}$ I $368.744 \mathrm{~nm})$ was examined. The distances between the laser target and glow discharge cathode vary from the center of the glow discharge cell outward, with the center of the cell defined as $0.0 \mathrm{~mm}$ for each. Because of the physical dimensions of the cathode and the laser target, it is impossible to have both at distance 0.0 simultaneously. As a result, the lowest cathode distance achievable was $4.0 \mathrm{~mm}$. 

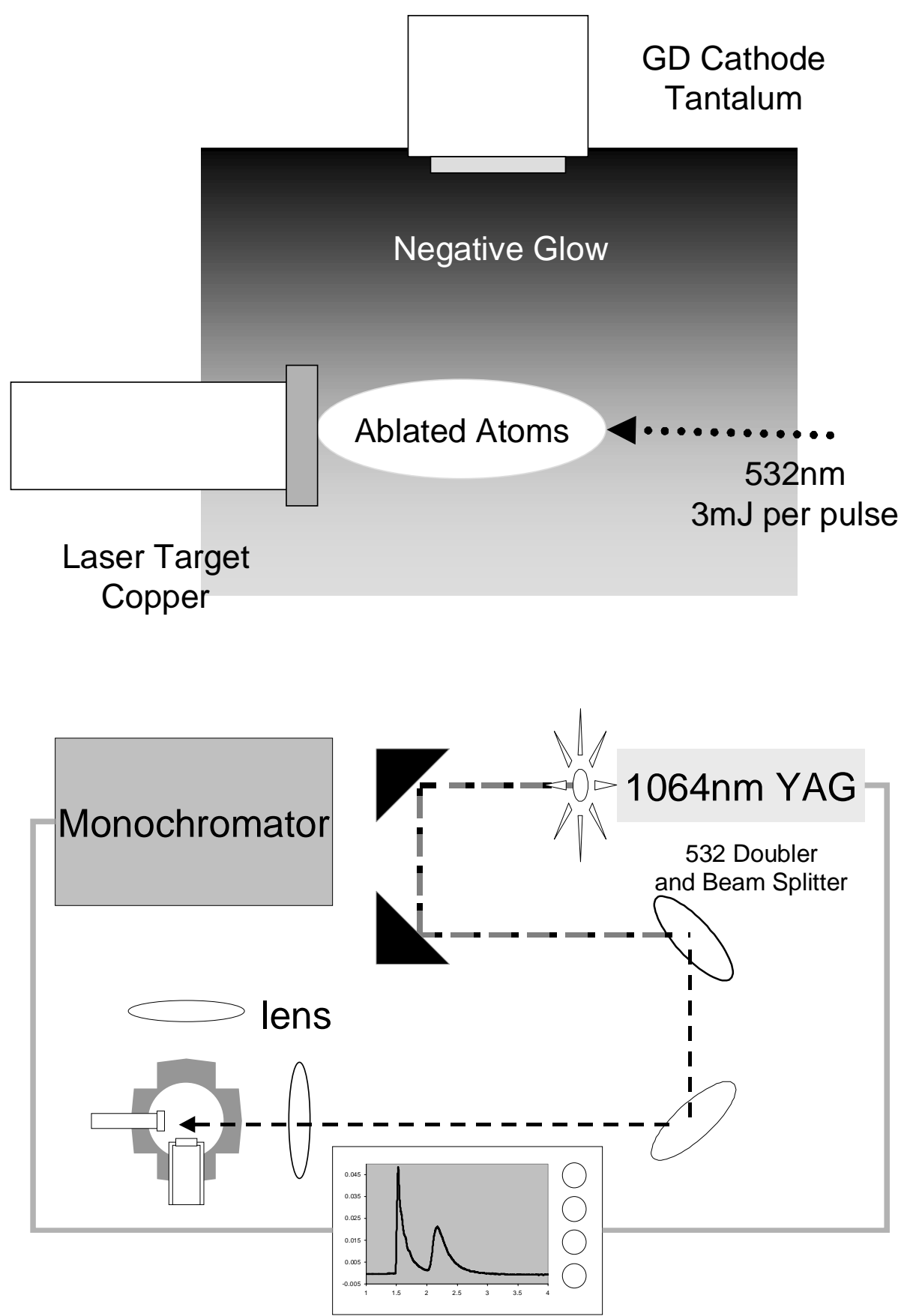

Figure 7.2.1.1 Experimental setup. Optical collection is from the center of the glow discharge cell and in the direction out of the plane of the paper. 


\subsection{Results and Discussion}

A spectrum of the glow discharge emission obtained using a copper cathode reveals two closely spaced emission lines at $368.655 \mathrm{~nm}$ (Cu II) and $368.744 \mathrm{~nm}(\mathrm{Cu} \mathrm{I})$. Given the bandpass of $0.7 \mathrm{~nm}$ used in our ablation experiments, both of these lines will be detected within our spectral window. The copper ion line, however is a factor of $10-20$ times weaker than the atom line during the pulse plateau region. During the pulse afterpeak region, the ion line is a factor of 100 times weaker than the atom line. Additionally, the atom line displays strong afterpeak behavior, while the ion line does not. We feel that the behavior observed during the laser ablation experiments is therefore solely from the atomic emission at $368.744 \mathrm{~nm}$. Excitation of this line likely occurs via Penning ionization of copper followed by ion-electron recombination to excite the atomic transition. ${ }^{12,54}$ Electron impact excitation may also contribute during the plateau region. ${ }^{12}$

Figure 7.3.1.1 illustrates the relative timing of the discharge and laser pulses and also demonstrates the differences observed in copper atom emission following ablation in the presence and absence of a glow discharge pulse. Figure 7.3.1.1a shows the $2.0 \mathrm{~ms}$ applied glow discharge square wave. Each delay is measured from the onset of the glow discharge pulse, with that onset being denoted as time $0 \mathrm{~ms}$. In Figure $7.3 .1 .1 \mathrm{~b}$ the laser is fired at $1.75 \mathrm{~ms}$ into the timing cycle, with no power applied to the glow discharge. The emission shown in Figure 7.3.1.1b comes from the laser-induced plasma pulse alone, and has two distinct features. First, there is an emission spike with some contribution from 
continuum emission plus a contribution from ablated copper liberated and excited by the 10 ns laser pulse.

a

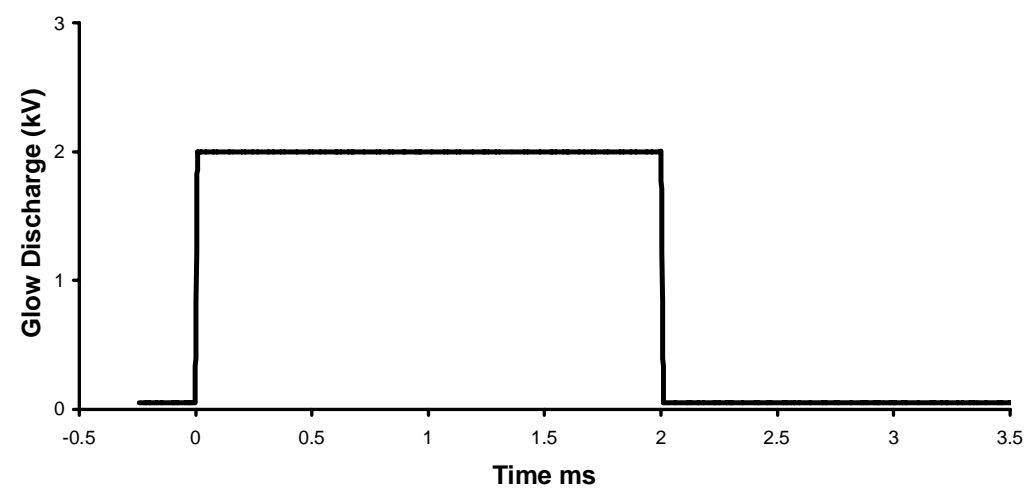

b

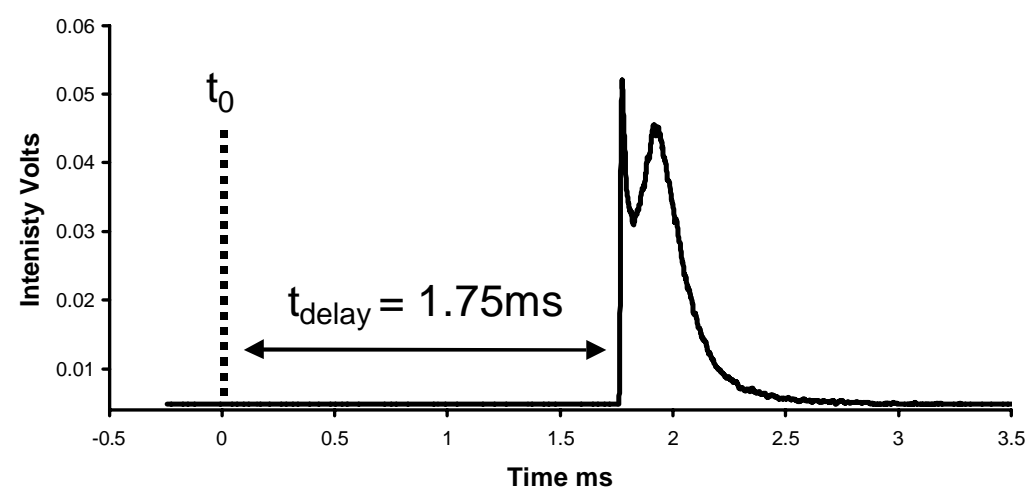

c

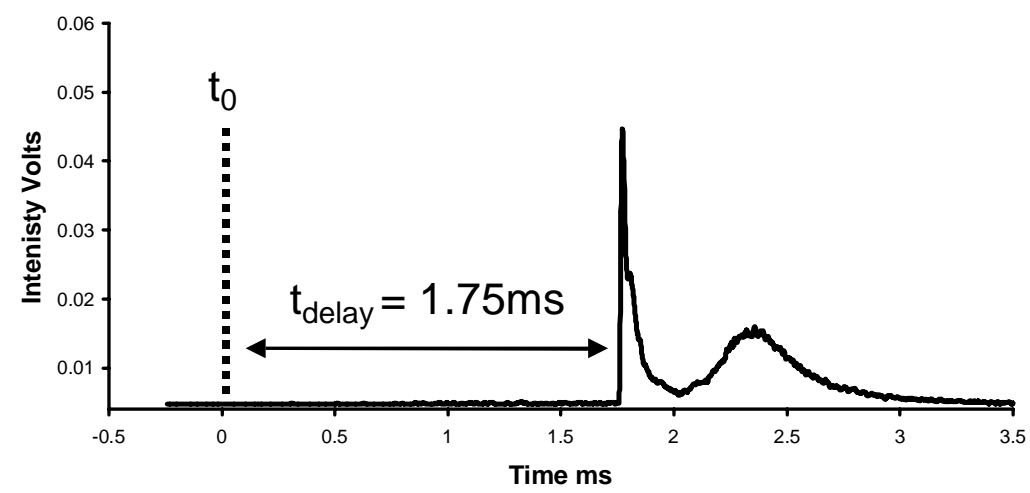

Figure 7.3.1.1 a) Temporal profile of the $2 \mathrm{~ms}$ applied glow discharge power pulse, b) Temporal profile of copper atom emission at $368.7 \mathrm{~nm}$, laser ablation only, no power to the glow discharge, and c) Temporal profile of copper atom emission at $368.7 \mathrm{~nm}$, during laser ablation with $2 \mathrm{kV}$ applied to the discharge. In both $b$ and $c$ the argon bath gas was at a pressure of 0.5 Torr. 
Second, decay of this initial spike is followed by a rise in intensity produced by the subsequent excitation of copper atoms from argon metastables formed by the laser breakdown of the argon medium. ${ }^{55}$ Figure $7.3 .1 .1 \mathrm{c}$ shows the copper atom emission from laser ablated material while the glow discharge is on, with the laser ablation pulse set at $1.75 \mathrm{~ms}$. Once again, the initial emission due to the laser-induced plasma is evident. However, the subsequent rise in intensity from the metastable population does not occur until $350 \mu$ s after glow discharge power termination. No emission at the detection wavelength is observed in the case where the discharge is pulsed, but the laser is not fired.

The emission profiles in Figure 7.3.1.2a, taken when the glow discharge is off, demonstrate that the argon metastables formed by the laser breakdown closely follow the laser delay position. The emission maximum from copper atoms excited by laser breakdown induced argon metastables appears, on average, $145 \mu$ s after the laser is fired, and decays over the next $400 \mu$ s. In Figure 7.3.1.2b, the $2.0 \mathrm{~ms}$ glow discharge was turned on and the laser was positioned at the same delays used in Figure 7.3.1.2a. The delay position of the laser can be verified by the rapid rise time in intensity from the emission of excited material coming from the laser pulse. However, the metastable population formed from the laser breakdown is no longer evident. Instead, copper atom emission coming from a metastable-induced population appears approximately $350 \mu$ s after the glow discharge pulse, and decays over the next $400 \mu \mathrm{s}$. This maximum is known as the glow discharge afterpeak, during which a 
surge in argon metastable atoms is formed by the recombination of argon ions and thermal electrons once power is terminated.

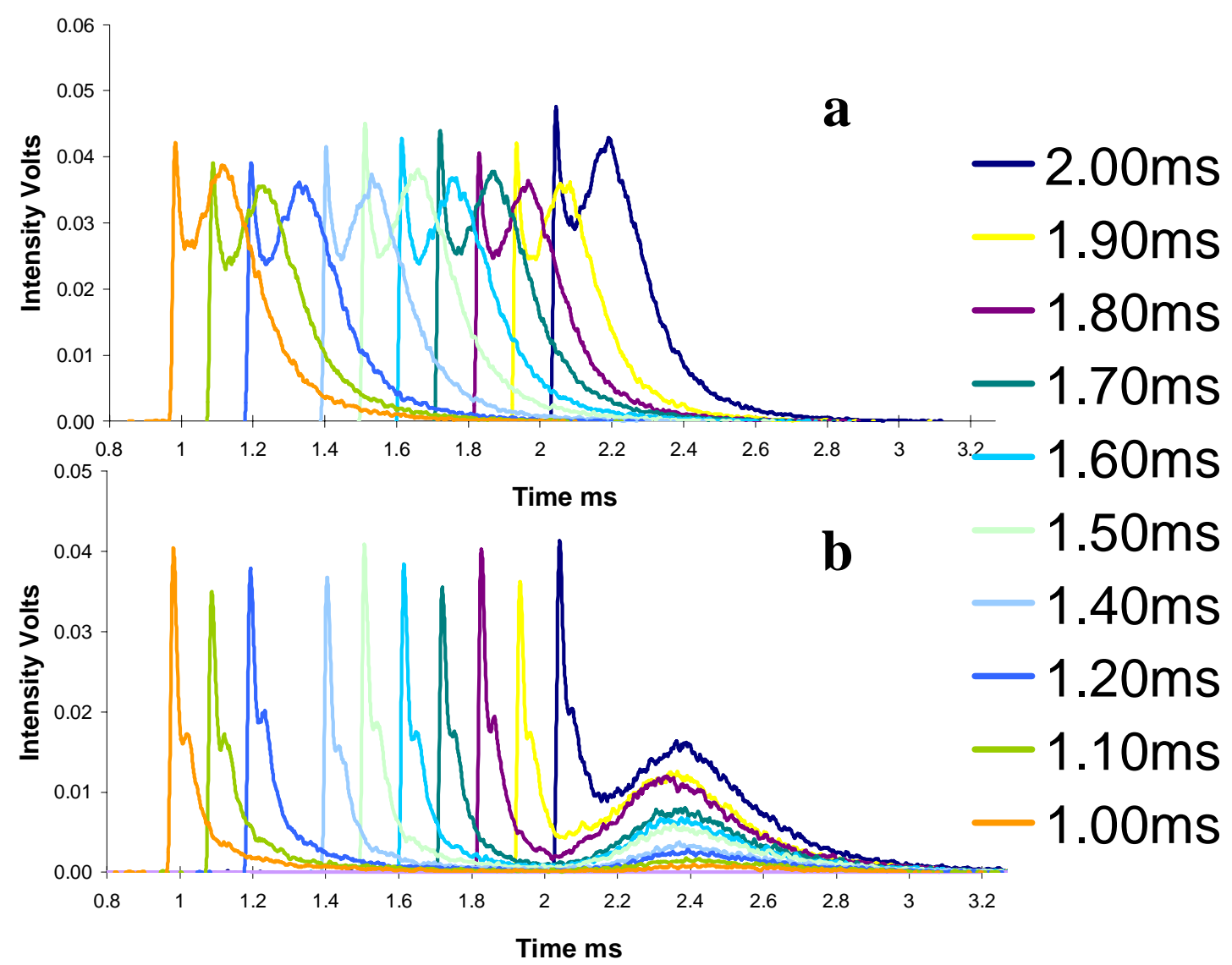

Figure 7.3.1.2 a) Temporal profiles of copper atom emission ( $368.7 \mathrm{~nm})$, for a series of laser delays with the glow discharge turned off. b) Copper atom emission $(368.2 \mathrm{~nm})$, for a series of delays with the glow discharge plasma turned on ( $2 \mathrm{kV}$ applied). In both $\mathrm{a}$ and $\mathrm{b}$ the argon bath gas was at a pressure of 0.5 Torr. The laser target was positioned at $4 \mathrm{~mm}$, with the auxiliary glow discharge cathode positioned at $10 \mathrm{~mm}$ from the center of the observation window. 
An important observation is that the long-lived copper atom emission arising from both the laser induced plasma and the glow discharge afterpeak display equivalent lifetimes of ca. $400 \mu \mathrm{s}$. This indicates a similar excitation mechanism for both populations - one arising from collisions with argon metastable atoms. This copper atom emission maximum occurs roughly at a fixed temporal position, independent of laser delay when the glow discharge is applied. This further suggests that the copper atom emission observed in Figure $3 \mathrm{~b}$ is due to the interaction of ablated material with argon metastables generated by the glow discharge, and not by those formed by the laser induced breakdown. In fact, copper atom emission generated by laser-induced metastables is not observed when the discharge is applied. We observe this behavior for laser delay times of up to (and including) $2.0 \mathrm{~ms}$. It is possible that during this time plasma species created by the glow discharge efficiently de-excite, or hamper the production of, the laser induced argon metastables before they can excite ablated copper atoms. For laser delay times greater than $2.0 \mathrm{~ms}$, power to the discharge plasma has been switched off, and discharge species responsible for de-exciting laser generated metastables are no longer present. As a result, laser-generated metastables now coexist with discharge-generated metastables, and it is not possible to separate copper excitation due to one process from the other. For this reason the laser is fired only within the $2.0 \mathrm{~ms}$ glow discharge pulse, so that only behavior associated with the discharge-generated metastables is observed in the afterpeak. An attempt to lower the laser power in 
hopes of ablating material without the accompanying laser-induced breakdown was unsuccessful.

Changes in afterpeak intensity and temporal position seen in Figure 7.3.1.3b are dependent on parameters that affect both transport of ablated material and diffusion of glow discharge plasma species. The excitation behavior observed arises from a convolution of the transport of ablated material with the diffusion of relevant plasma species. Optimizing Penning excitation of ablated material involves its transport into a metastable rich plasma region when the argon metastable population is at a maximum. The exact position in the glow discharge plume where this occurs depends on glow discharge conditions such as the working gas pressure and applied power. Ablated material is carried into the plasma via directed transport behind a shock wave front. ${ }^{56,57,58}$ Changing pressure not only results in varying transport times of ablated material, but also changes the spatial position of the metastable population maximum coming from the glow discharge plume. Therefore, there should be a unique optimum laser target to glow discharge cathode distance and laser firing time for each pressure studied. This represents a two-fold transport problem, for which two sets of experiments were performed.

\subsubsection{Part I. Transport of Ablated Material}

First, the transport of the ablated population was examined at varying laser target distances and introduction times for a range of pressures. At each pressure, the position of the metastable population was fixed by holding the glow 
discharge cathode $10.0 \mathrm{~mm}$ from the center of the glow discharge. The laser target was positioned from 0.0 to $10.0 \mathrm{~mm}$ from the center of the cell in $1.0 \mathrm{~mm}$ increments. At each target position, the laser delay was set at 2.0, 1.9, 1.8, 1.5, 1.3, and $1.0 \mathrm{~ms}$ into the glow discharge timing sequence. Atom emission profiles were then recorded at working gas pressures ranging from 0.5 to 0.8 torr. Figure 7.3.1.2 $\mathrm{b}$ shows the results of experiments performed for a fixed target position and pressure, at various laser delay times. Each emission profile shows an increase in afterpeak intensity as the delay time is moved closer to the afterpeak position. Figure 7.3.1.4 shows the dependence of afterpeak intensity on target distance, obtained at a fixed delay time and pressure. For both pressures shown, the intensity of the afterpeak decreases as target distance increases.

At a fixed distance and pressure, changing the laser delay permits introduction of the ablated atom population into the negative glow throughout the glow discharge pulse cycle. We sought to optimize sample introduction to maximize spatial overlap of ablated material with the argon metastable population produced by the glow discharge. Depending on pressure and cathode position, the maximum population will occur in line with the ablated material at a time $200-400 \mu$ s after termination of the glow discharge power. This requires the timing of the ablation pulse to produce a material plume that arrives in this excitation/ionization region during the $200-400 \mu$ s timeframe after termination of the discharge. The data shown in Figure 7.3.1.2b demonstrates that we are able to adjust the timing of our sampling in a way that will improve signal intensity. 
Optical flight-time measurements of laser ablated Cu have shown that, for a fixed observation position, a maximum should appear in the distribution of ablated material as a function of target distance. ${ }^{59}$ Similarly, as the timing of the ablation pulse is swept through the glow discharge cycle, a maximum overlap between the ablatant and the metastable region of the plasma should occur. We did not observe this behavior. This may be due to the ablated material moving rapidly enough that the bulk of the ablated material has passed through the zone where highest overlap with argon metastables would occur before the metastables have had time to form or diffuse into this region. Imaging studies of laser ablation have demonstrated that the bulk of the ablated material undergoes directed transport away from the target surface, while a lower concentration distribution trails behind. ${ }^{58,60}$ The leading edge of material is known to initially travel at supersonic speeds, with velocities ranging from 2 to $20 \mathrm{~km} / \mathrm{sec}^{56,61,62}$ Although measurements have shown that this initial velocity drops rapidly to subsonic speeds, a conservative estimated average speed of $1 \mathrm{~km} / \mathrm{sec}$ will move the leading edge of material $1 \mathrm{~cm}$ in $10 \mu \mathrm{s}$. However, it has been shown that a lower concentration distribution of material will trail the bulk distribution by up to several milliseconds. ${ }^{58}$ This leads to the following scenario: Setting the laser delay at $2 \mathrm{~ms}$ will result in the leading edge of ablated material passing through the plasma interaction region 200 to $300 \mu$ s before the discharge metastable population maximizes in the interaction region. It is therefore excitation of the trailing distribution of material that gives rise to our observed afterpeak signal. 
The above analysis is in agreement with our observation (Figure 7.3.1.3b) that signal intensity drops as the laser delay is adjusted to earlier ablation times. Moving to earlier ablation times will result in excitation of progressively lower concentrations of the ablation tail. Earlier ablation times may also produce decreased signal due to increased time for condensation of ablated material into particles that may not be excited in the plasma. Given our low working pressure, however, this may not be a significant contribution, ${ }^{58}$ but it will be an important area for future study. In terms of signal optimization, our results suggest it should be possible to significantly increase signal intensity by adjusting the laser delay to times following termination of the glow discharge. This will be the subject of future investigations.

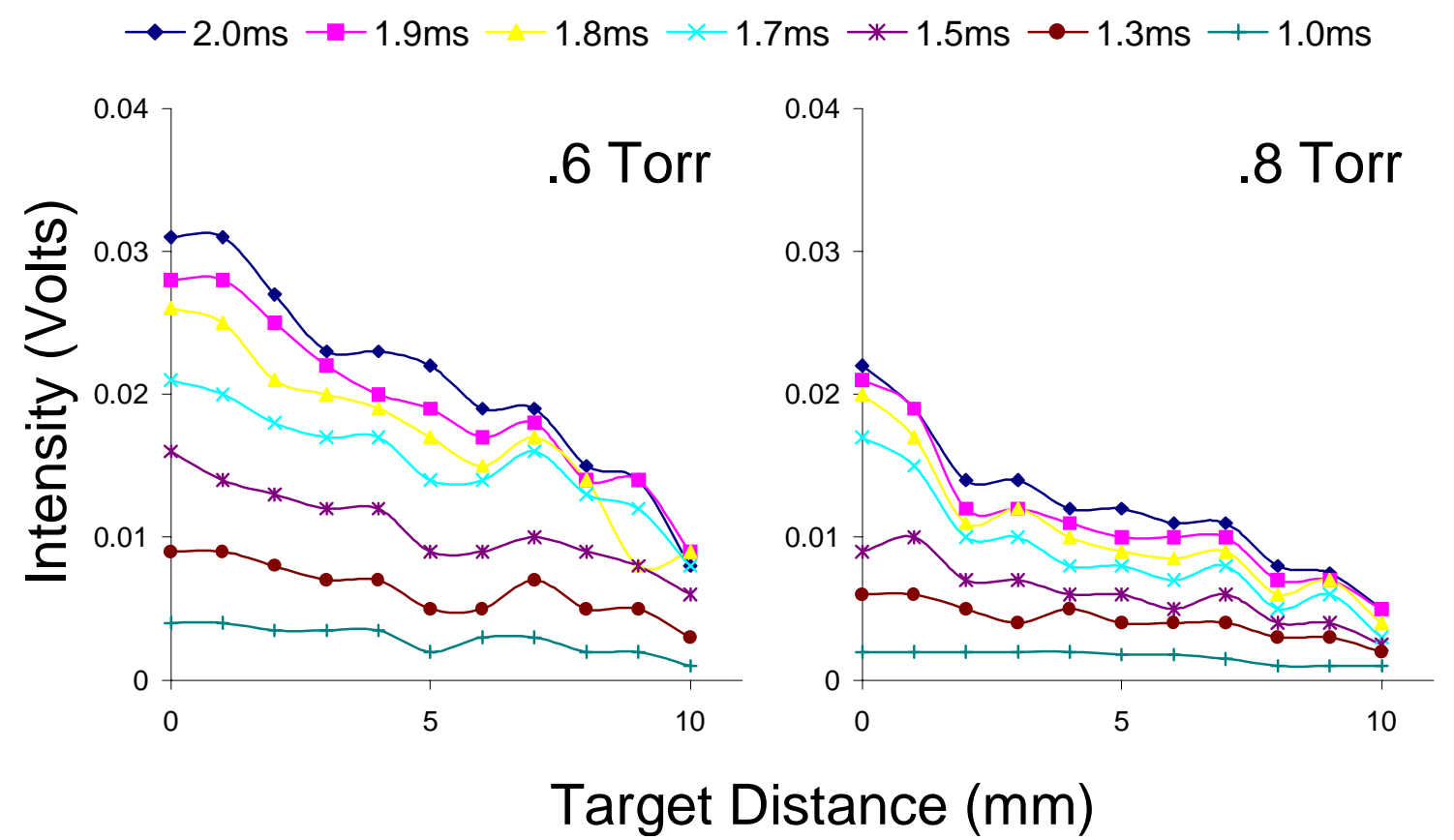

Figure 7.3.1.3Afterpeak intensity dependence on copper target distance at pressures of 0.6 and 0.8 torr. Individual traces display dependence at a given laser delay time. Cathode distance fixed at $10 \mathrm{~mm}$. 
Optimal signal intensity is obtained by minimizing the distance between the laser target and the plasma interaction region as demonstrated in Figure 4. This is in agreement with previous results for laser ablation in an argon atmosphere, in which the quantity of ablated material reaching the observation region displays an exponential decay with increasing distance and pressure. ${ }^{61}$ This is a result of increased scattering of the material by the Ar fill gas for greater pathlengths and higher pressure. For observations at higher pressure the spatial distribution should decay faster with distance than at lower pressures. This is seen in Figure 7.3.1.4, where the distribution has already rapidly decayed to a flattened tail at 0.8 torr, while the distribution is still decaying at 0.6 torr. Therefore, the distance between the laser target and plasma interaction region must be minimized to avoid loss of material.

\subsubsection{Part II. Glow Discharge Metastable Distributions}

As discussed previously, not only is the timing of the laser ablation important in terms of determining when the ablated material arrives in the discharge plasma, but it is also important that the material arrives when the discharge metastables are maximized in this zone. This aspect of timing is dependent upon diffusion processes occurring within the plasma. We performed further investigations that examined the spatial and temporal position of the metastable maximum under different working gas pressures. This was accomplished by moving the glow discharge cathode away from the laser target while the target is fixed at a distance of $0.0 \mathrm{~mm}$. The laser delay was set at $1.5 \mathrm{~ms}$ into the glow discharge pulse cycle to minimize contribution of copper emission originating 
from collisions with laser-induced metastables. Temporal emission profiles were collected over a pressure range of 0.5 to 0.8 torr, and at cathode distances spanning 4 to $20 \mathrm{~mm}$ (in $2 \mathrm{~mm}$ increments). Figure 7.3.2.1 demonstrates the changes of the copper atom emission profile under increasing pressure and cathode-target distances. Figures 7.3.2.2 and 7.3.2.3 illustrate both the changes in the afterpeak signal intensity and in temporal position as distance is increased. As pressure is increased, the overall intensity of the afterpeak diminishes, while the temporal position of its maximum increases. These two trends are explained by considering the effects of collision density on both the ablated atom and the glow discharge plasma metastable population.

In general, as the pressure is increased, the glow discharge plume and darkspace shrink around the cathode. Increasing pressure increases the collision density, allowing electrons to thermalize and recombine with argon ions closer to the cathode surface. This moves the metastable population maximum closer to the cathode. This has also been observed in fluorescence measurements ${ }^{63}$ and has been predicted through modeling of a steady state discharge. ${ }^{64}$ Figure 7.3.2.2 is a plot of afterpeak maximum intensity as a function of cathode distance for the pressure range studied. At greater cathode distances, the afterpeak intensity should increase to a maximum, then decrease as the region of maximum population for metastables is swept through our viewing region. This behavior is visible at 0.5 torr, but is not observed at greater pressures. The smallest cathode distance studied was $4 \mathrm{~mm}$, thus, at higher pressures ( $>0.5$ torr) the afterpeak metastable population may maximize within 
- 0.5 Torr

- 0.6 Torr

0.7 Torr

0.8 Torr

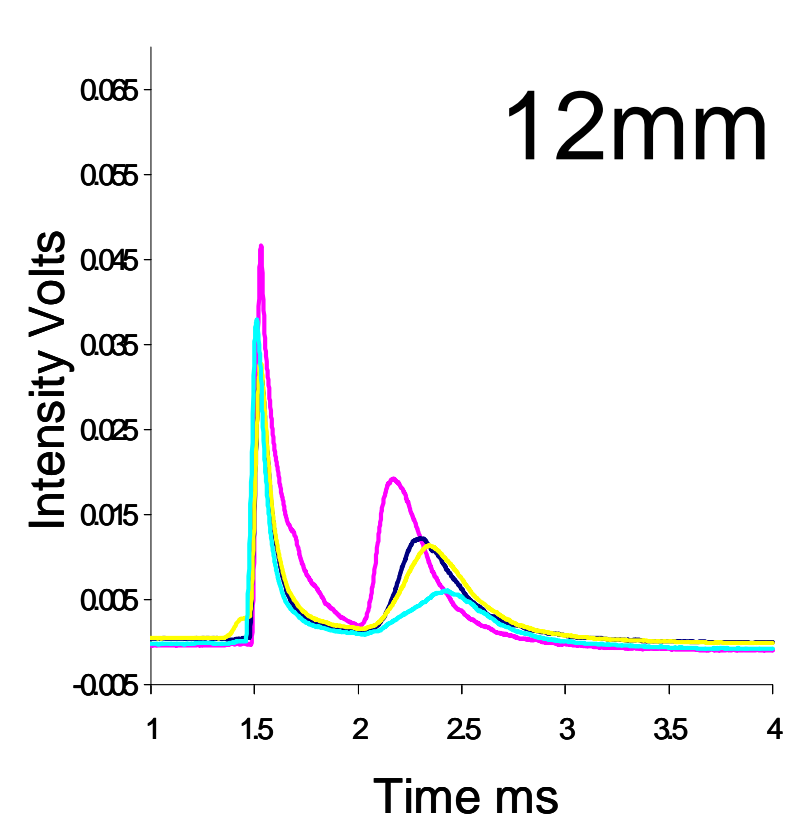

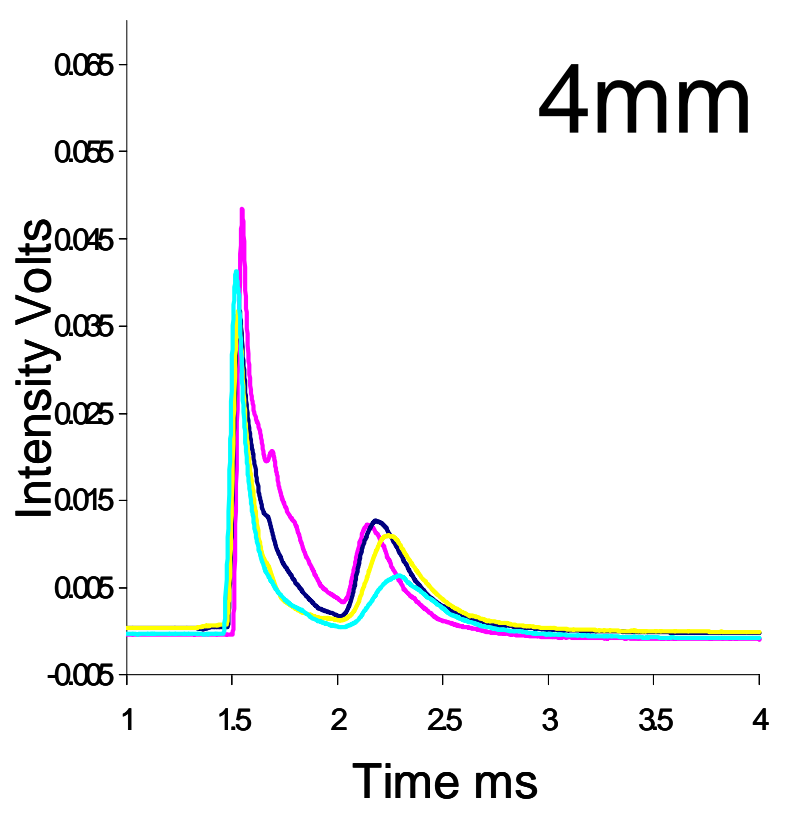

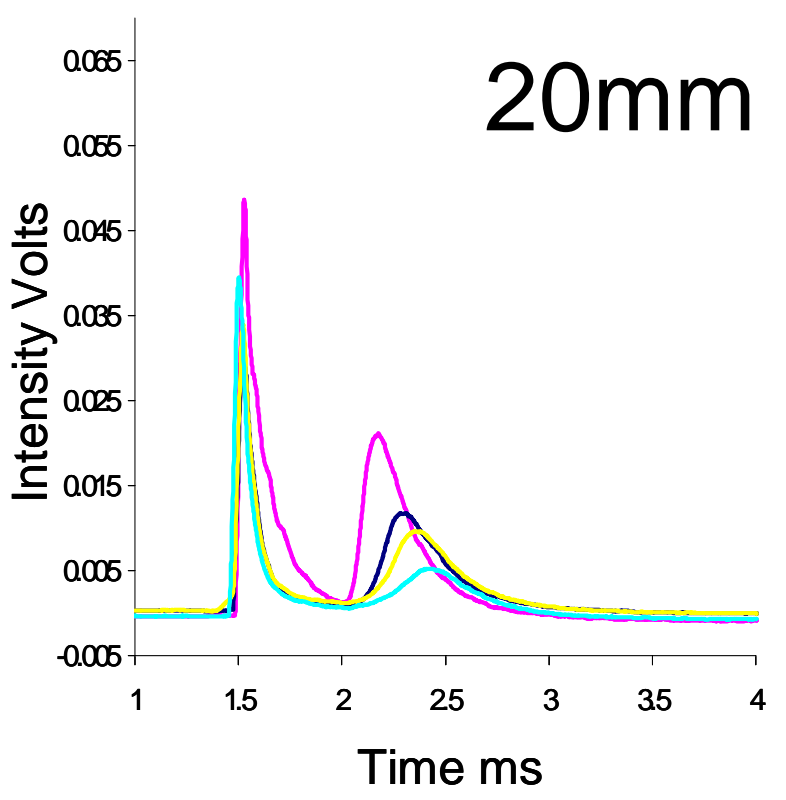

Figure 7.3.2.1 Temporal profiles of copper atom emission $(368.2 \mathrm{~nm})$ for a series of pressures at 3 given cathode distances. Laser delay of $1.5 \mathrm{~ms}$ with a $2 \mathrm{~ms}$, $1 \mathrm{kV}$ applied power pulse. Target distance fixed at $0 \mathrm{~mm}$ 
the boundary imposed by the physical dimensions of the laser target and glow discharge cathode. As pressure increases, the distance and diffusion time needed for the metastables produced by the glow discharge to reach the region of interaction with the ablated material increases (see Figure 7.3.2.3). Therefore, as pressure is increased, the temporal position of the afterpeak maximum moves to longer times during the pulse cycle for all distances studied. This is due to increased collision density, which slows the diffusion of glow discharge metastables. Also, metastables produced by the glow discharge must now travel longer distances, since the metastable population now forms closer to the cathode surface. Both diffusion processes result in a temporal shift of the afterpeak maximum to longer times, as illustrated in Figure 7.3.2.1

Loss in afterpeak signal intensity as the pressure is increased is illustrated in Figure 7.3.2.1. Hess noted a similar phenomenon in his investigations using a steady state discharge. ${ }^{47}$ This behavior is an interesting contrast to that observed for glow discharge alone, for which Penning excitation increases with increasing pressure. ${ }^{10}$ The cause of this behavior in our experiments is related to the ablation and material transport process. As noted previously, as pressure is increased, more ablated material is scattered away from the detection region and does not interact with the discharge metastables. Another contributing factor is the screening of the trailing portion of the ablation pulse through inverse bremsstrahlung absorption by the plasma created by its leading edge. It has been demonstrated that absorption by plasma screening increases with increasing pressure. ${ }^{65}$ Although this is a stronger effect for picosecond laser 


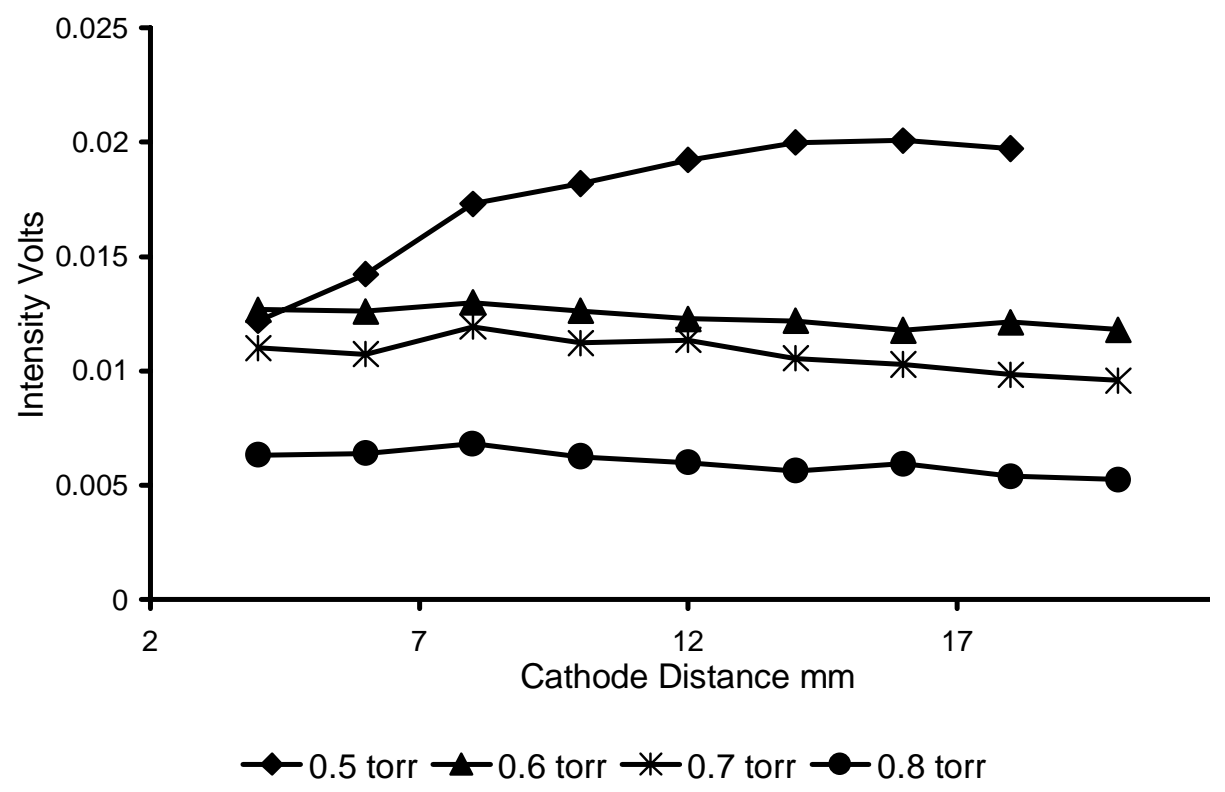

Figure 7.3.2.2 Copper atom $(368.2 \mathrm{~nm})$ afterpeak intensity maximum intensity as a function of cathode distance for a series of glow discharge working pressures. Laser pulse delay of $1.5 \mathrm{~ms}$ with a $3 \mathrm{~ms}, 1 \mathrm{kV}$ applied glow discharge pulse. Copper laser target positioned at $0 \mathrm{~mm}$.

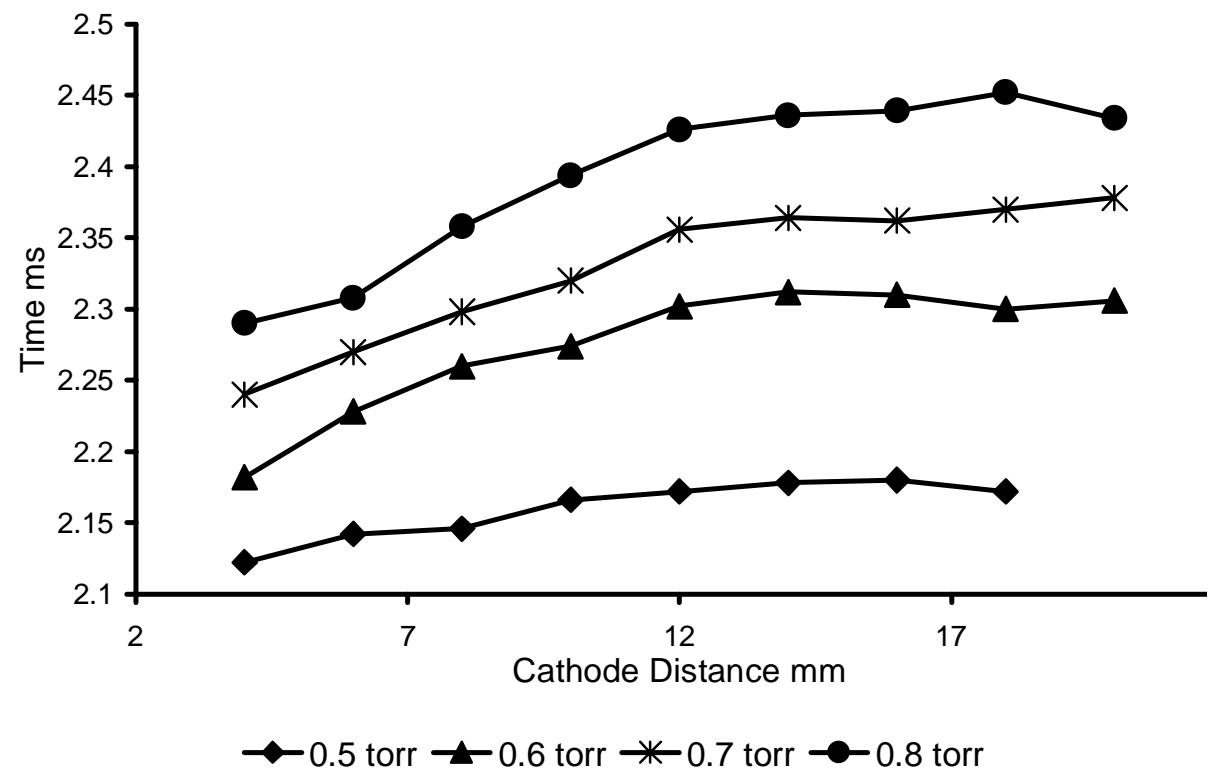

Figure 7.3.2.3 Copper atom (368.2nm) afterpeak temporal position as a function of cathode distance for a series of glow discharge working pressures. Laser pulse delay of $1.5 \mathrm{~ms}$ with a $3 \mathrm{~ms}, 1 \mathrm{kV}$ applied glow discharge pulse. Copper laser target positioned at $0 \mathrm{~mm}$. 
pulses, it also contributes to decreased sampling efficiency for nanosecond ablation pulses. Given the opposing trends with pressure between metastable generation by the glow discharge and ablation sampling and transport efficiency, an optimum pressure must be found. Our data demonstrates an increase in intensity by decreasing pressure to 0.5 torr. Further reduction of pressure below 0.5 torr yields no appreciable glow discharge metastable population.

Experimental focus in this study was on the interaction of ablated material with argon metastables during the afterpeak time region. This is the relevant excitation region for future applications to molecular analysis, in that it is Penning ionization during this time that will generate whole molecular ions. However, it will be equally important to have the capability to generate molecular fragments. Fragmentation will occur most efficiently by electron impact ionization during the prepeak and plateau regions of the pulse cycle. Observations of the pulse plateau revealed only weak copper atom emission at all pressures studied. During the plateau, ionization and excitation occurs via a combination of electron impact and Penning ionization. The lack of copper atom emission suggests that excitation during this temporal regime is highly dependent on both distance and pressure. As pressure and distance increases, plateau intensity decreases. Increasing distance and/or pressure will decrease the population of fast electrons available for ionization/excitation through electron impact. Figures 7.3.2.4a and b show clear examples of this phenomenon. In Figure 7.3.2.4c, at a pressure of 0.5 Torr, the plateau intensity is strongest near the cathode surface, and drops off with distance from the cathode. This behavior is opposite to that observed for 


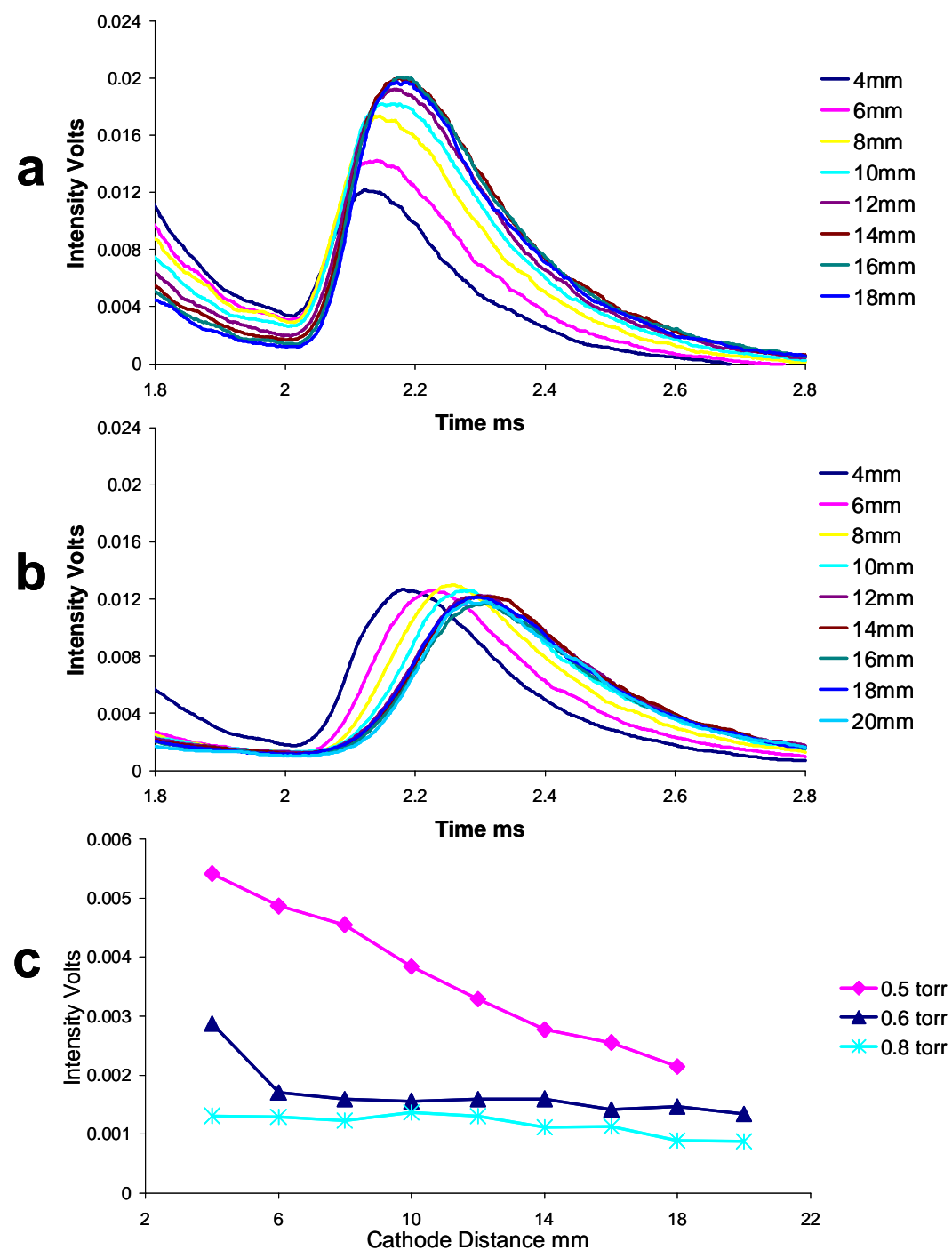

Figure 7.3.2.4 Expanded view of copper atom emission (368.7 $\mathrm{nm}$ ) profiles at increasing cathode distances during the afterpeak time regime at a pressure of $\mathbf{a}) 0.5$ and b) 0.6 torr. Laser pulse delay of 1.5 ms with a $1 \mathrm{kV}$ applied glow discharge pulse, torr, with the copper laser target positioned at $0 \mathrm{~mm}$. c) Measured plateau intensity as a function of cathode distance (1.90 ms into the pulse cycle) 
the adjacent afterpeak, Figure 7.3.2.4a. By contrast, a slight increase in pressure to 0.6 torr, Figure $7.3 .2 .4 \mathrm{~b}$, results in a much lower plateau intensity that is constant at all distances, Figure 7.3.2.4c. Detailed investigations of plateau and prepeak regimes will be the subject of future investigations.

\subsection{Conclusions}

Detection of laser ablated material following excitation by metastable atoms produced by a pulsed secondary glow discharge during its afterpeak region has been demonstrated. Optimized detection is limited to a small pressure and ablation timing window. As pressure is increased, ablated material reaching the Ar metastable population is reduced through scattering and plasma screening effects; further reduction in pressure, however, reduces the overall metastable population. Optimized timing of sample introduction into the plasma was found to be dependent on plasma diffusion processes, as well as on laser firing time. The diffusion processes are also highly sensitive to changes in pressure. Future investigations will focus on use of ablation pulses nearer in time to the after-peak region. Subsequent problems associated with discriminating between overlap in laser-induced versus plasma-induced excitation will be addressed by increasing detection sensitivity and lowering the laser ablation energy. Future experiments will employ a microsecond glow discharge source to increase plasma density, increasing both glow discharge metastable production and electron ionization. The application of this hyphenated source will be employed in time-gated mass spectrometric detection schemes for 
the characterization of elemental, structural, and molecular information of polymers, nonconductors, and biomolecules. 


\section{References:}

1 S.E. Frost, "Basic Teachings of the Great Philosophers", Dell Publishing Inc., 1942.

${ }^{2}$ L. Lenderman and D. Teresi," The God Particle", Dell Publishing Inc., 1993.

3 J.A. Broekaert, Applied Spectroscopy, 1995, 49, 12 A.

${ }^{4}$ H.J. Svec, International J of Mass Spec and Ion Processes, 1985, 66, 3.

${ }^{5}$ J.W. Coburn and E. Kay, Appl. Phys. Lett. 1971, 46, 1236.

${ }^{6}$ N. Jakubowski, D. Stuewer, and G. Toelg, Spectrochim. Acta, 1991, 46B, 155.

${ }^{7}$ K.R. Hess, R.K. Marcus, F.L. King, and W.W. Harrison. Anal. Chem., 1986, 58, $341 \mathrm{~A}$.

${ }^{8}$ F.L. King and W.W. Harrison Mass Spectrom Reviews, 1990, 9, 285.

${ }^{9}$ F.L King, J. Teng, and R.E. Steiner, J. Mass Spectrom. 1995, 30, 1061.

${ }^{10}$ R. L. Smith, D. Serxner and K. R. Hess, Anal. Chem. 1989, 61, 1103.

${ }^{11}$ R.K. Marcus, "Glow Discharge Spectroscopies", R.K. Marcus, Ed.; Plenum

Press: New York, 1993

${ }^{12}$ C. Pan and F.L. King, Anal. Chem, 1993, 65, 735.

${ }^{13}$ C. Pan and F.L. King, App. Spectr, 1993, 47, 300.

${ }^{14}$ D. Pollman, K. Ingeneri, and W.W. Harrison, J. of Anal Atom Spect, 1996, 11, 849.

${ }^{15}$ W. Grimm, Spectrochim. Acta, 1968, 23B, 443.

${ }^{16}$ J.A. Kingler, C.M. Barshick, and W.W. Harrison, Anal. Chem. 1991, 63, 2571. 
${ }^{17}$ D.C. Duckworth, D.H. Smith, and S.A. Mcluckey, Anal. Atom. Spectrom. 1997, 43, 12.

${ }^{18}$ R.E. Steiner, C.L. Lewis, and F.L., King, Anal. Chem. 1997, 69, 1715.

${ }^{19}$ W. Hang, C. Baker, B.W. Smith, J.D. Winefordner, W.W. Harrsion, J. Anal. Atom. Spectrom, 1997, 12, 143.

${ }^{20}$ J.A. Kingler, P.J. Savickas, and W.W. Harrison, J. Am. Soc. Mass. Spectrom., 1990, 1, 138.

${ }^{21}$ W. Hang, W.O. Walden, and W.W. Harrison, Anal. Chem., 1996, 68, 1148.

${ }^{22}$ C.L. Lewis, E.S. Oxley, C. Pan, R.E. Steiner, and F.L. King, Anal Chem., 1999, $71,230$.

${ }^{23}$ V. Majidi, M. Moser, C. Lewis, W. Hang, and F.L. King, J. Anal. At. Spectrom., 2000, 15, 19.

${ }^{24}$ P.A. Janson, Deconvolution with Applications in Spectroscopy, Academic Press, Inc., Orlando Florida. 1984

${ }^{25}$ D.A Scott and A.V. Phelps, Phys. Rev. A, 1991, 43, 3043.

${ }^{26}$ A. Bogaerts and R. Gijbels, Spectrochim. Acta., 2000, 55B, 279.

${ }^{27}$ W.L. Wiese and G.A. Martin, "Wavelengths and Transition Probabilities for Atoms and Atomic lons", NSRDS-NBS, 1980.

${ }^{28}$ B. Chapman, "Glow Discharge Processes, Sputtering and Plasma Etching", John Willey and Sons, NY, 1980.

${ }^{29}$ F.P. Ferria and H.G.C. Human, Spectrochim. Acta., 1981, 36B, 215.

${ }^{30}$ K. Hoppstock and W.W. Harrison, Anal. Chem., 1995, 67, 3167. 
${ }^{31}$ A. Bogaerts, E. Wagner, B.W. Smith, J.D. Winefordner, D. Pollman, W.W. Harrison, R. Gijbels, Spectrochimica Acta Part B, 1997, 52, 205.

${ }^{32}$ A. Bogaerts and R. Gijbels, Anal. Chem., 1996, 68, 2676.

${ }^{33}$ E.B.M. Steers and J. F. Fielding, J. Anal. At. Spectrom., 1987, 2, 239.

${ }^{34}$ K. Wagatsuma and K. Hirokawa, Spectrochimica Acta Part B, 1991, 46, 269.

${ }^{35}$ J. Teng, R.E. Steiner, and F.L. King, J. Am. Soc. Mass Spectrom. 1995, 30, 1061.

${ }^{36}$ R.E. Steiner, C.L. Lewis, and V. Majidi, J. Analyt. Atom. Spect.1999, 9, 1537.

${ }^{37}$ R.K. Marcus, F.L. King and W.W. Harrison, Anal. Chem. 1986, 58, 972-974.

${ }^{38}$ R. Jager, J.S. Becker, H.J. Dietze, and J.A.C. Broekart, Int J. of Mass Spectrom. and Ion Proces, 1997, 171, 183-89

${ }^{39}$ R.C. Deng, and P. Williams, Anal. Chem, 1994, 66, 1890-1896

${ }^{40}$ F.L. King, A.L. McCormack, and W.W. Harrison, J. Anal. At. Spectrom. 1988, 3, 883.

${ }^{41}$ N. Jakubowski and D. Stuewer, Fresenius Z. Anal. Chem. 1989, 335, 680.

${ }^{42}$ C. Pan and F.L. King, J. Am. Soc. Mass Spectrom. 1993, 4, 727.

${ }^{43}$ A. Raith, and R.C. Hutton, J. Anal. At. Spectrom. 1992, 7, 943.

${ }^{44}$ R.E. Russo, Appl. Spectrosc. 1995, 49, 14A.

${ }^{45}$ E.R. Denoyer, K.J. Fredeen, and J.W. Hager, Anal. Chem. 1991, 63, 445A.

${ }^{46}$ Y. lida, Spectrochimica Acta 1990, 45B, 427.

${ }^{47}$ K.R. Hess, "Laser-Based Methods", in Glow Discharge Spectroscopies, R.K. Marcus, ed.; (Plenum Press, NY, 1993), Chap. 10. 
${ }^{48}$ K.R. Hess and W.W. Harrison,"Laser Ablation and lonization Studies in a Glow Discharge", in Lasers and Mass Spectrometry, D.M. Lubman, ed. (Oxford Univ. Press, NY, 1990), Chap. 9.

${ }^{49}$ D. Figg and M.S. Kahr, Appl. Spect., 1997, 51, 1185.

${ }^{50}$ D.J. Figg, J.B. Cross and C. Brink, Appl. Surf. Sci., 1998, 127-129, 287.

${ }^{51}$ J. Teng, C.M. Barshick, D.C. Duckworth, S.J. Morton, D.H. Smith, and F.L. King, Appl. Spect., 1995, 49, 1361.

${ }^{52}$ D.C. Duckworth and R.K. Marcus, Anal. Chem. 1989, 61, 1789.

${ }^{53}$ F.L. King and W.W. Harrison, Int. J. Mass Spectrom. Ion Processes 1989, 89, 171.

${ }^{54}$ P.B. Farnsworth, J.P. Walters, Spectrochimica Acta 1982, 37B, 773.

${ }^{55}$ M.R. Joseph, N. Xu, N., and V. Majidi, Spectrochimica Acta 1994, 49B, 89.

${ }^{56}$ I.B. Gornushkin, M. Clara, B.W. Smith, J.D. Winefordner, U. Panne, and R. Niessner, Spectrochimica Acta, 1997, B52, 1617.

${ }^{57}$ W.S. Budi, H. Suyanto, H. Kurniawan, M.O. Tjia, and K. Kagawa Appl. Spect. 1999, 53, 719.

${ }^{58}$ A.D. Sappey and T.K. Gamble, J. Appl. Phys. 1992, 72, 5095.

59 J.D. Wu, Q. Pan, and S.C. Chen, Appl. Spect.1997, 51, 883.

${ }^{60}$ D.K. Zerkle and A.D. Sappey, IEEE Trans.Plasma Sci. 1996, 24, 37.

${ }^{61}$ X. Yang, Y. Tang, X. Liu, and Q. Qin, Appl. Spect., 1999, 53, 278.

${ }^{62}$ P.-N. Wang, Q. Pan, N.H. Cheung, and S.-C. Chen, Appl. Spect., 1999, 53, 205. 
${ }^{63}$ A. Bogaerts, R.D. Guenard, B.W. Smith, J.D. Winefordner, W.W. Harrison, and R. Gijbels, Spectrochimica Acta B, 1997, 52, 219.

${ }^{64}$ A. Bogaerts and R. Gijbels, Physical Rev. A, 1995, 52, 3743.

${ }^{65}$ X.L. Mao, W.-T. Chan, M.A. Shannon, and R.E. Russo, J. Appl. Phys., 1993, 74, 4915. 
Vita

\section{Cris Lee Lewis}

Department of Chemistry

West Virginia University, Morgantown WV 26505

Phone 304-293-3060 ext. 4220

Fax 304-293-4904

eMail: clewis@wvu.edu

\section{EDUCATION \\ Doctorate (2000) \\ Analytical Chemistry \\ West Virginia University \\ Morgantown, WV \\ Masters of Science (1998) \\ Analytical Chemistry \\ West Virginia University \\ Morgantown, WV \\ Bachelors of Science \\ Chemistry (1994) \\ West Virginia Institute of Technology \\ Montgomery, WV}

\section{PROFESSIONAL EXPERIENCE}

1994-1998 Chemistry Research Assistant, West Virginia University

1998-1999 Chemistry Research Assistant, Los Alamos National Laboratory

1999-2000 Chemistry Research Assistant, West Virginia University

\section{AWARDS}

1995 Outstanding First Year Teaching Assistant, Chemistry Department 2000 Outstanding Advanced Teaching Assistant, Chemistry Department

\section{TEACHING}

Teaching of General Chemistry Laboratory (8 Semesters)

Teaching of Quantitative Laboratory (1 Semester)

Teaching of Instrumental Analysis Laboratory (1 Semesters) 


\section{PUBLICATIONS}

R. E. Steiner, C. L. Lewis, and F. L. King, "Time-of-Flight Mass Spectrometry With a Pulsed Glow Discharge lonization Source"; Analytical Chemistry; 1997, 69, 1715.

C.L. Lewis, E.S. Oxley, C.K. Pan, R.E. Steiner, and F.L. King, "Determination of ${ }^{40} \mathrm{Ca}^{+}$in the Presence of ${ }^{40} \mathrm{Ar}^{+}$: An Illustration of the Utility of Time-gated Detection in Pulsed Glow Discharge Mass Spectrometry"; Analytical Chemistry; 1999, 71, 230.

R. E. Steiner, C. L. Lewis, and V. Majidi, "Consideration of a Millisecond Pulsed Glow Discharge for Concurrent Elemental and Molecular Analysis"; Journal of Analytical Atomic Spectrometry, 1999, 14, 1537.

Vahid Majidi, Matt Moser, Cris Lewis, Wei Hang, Fred. L. King, "Explicit Chemical Speciation by Microsecond Pulsed Glow Discharge Time-of-Flight Mass Spectrometry: Concurrent Acquisition of Structural, Molecular and Elemental Information"; Journal of Analytical Atomic Spectrometry, 2000, 15, 19.

Cris Lewis, Stephen K. Doorn, David M. Wayne, Fred L. King, and Vahid Majidi, "Characterization of a Pulsed Glow Discharge Laser Ablation System Using Optical Emission"; Accepted Applied Spectroscopy August 2000

Cris L. Lewis, Glen P. Jackson, Stephen K. Doorn, Vahid Majidi and Fred L. King, "Spectral, Spatial, and Temporal Characterization of a Millisecond Pulsed Glow Discharge: Analyte Emission and Ionization", Submitted to Spectrochemica Acta B.

Glen P. Jackson, Cris L. Lewis, Stephen K. Doorn, Vahid Majidi and Fred L. King, "Spectral, Spatial, and Temporal Characterization of a Millisecond Pulsed Glow Discharge: Argon Atom Metastables", Submitted to Spectrochemica Acta B. 


\section{CONFERENCE PRESENTATIONS}

C.L. Lewis, R.E. Steiner, E. Oxley, F.L. King, "Elimination of Isobaric Interferences Using Pulsed RF and DC Glow Discharge lonization Sources," presented at the 1997 Meeting of the Federation of Analytical Chemistry and Spectroscopy Societies, Providence, RI, October 26-30, 1997.

C.L. Lewis, E. Oxley, F.L. King, "Exploitation of Kinetic Energy Variations in Modulated Analytical Glow Discharges," presented at the Forty-sixth ASMS Conference on Mass Spectrometry and Allied Topics, Orlando, FL, May 31 June 5, 1998.

Cris Lewis, Wei Hang, Steve Doorn, Dave Wayne, Vahid Majidi, Fred King, "Characterization of a Pulsed Glow Discharge Laser Ablation System for Direct Chemical Analysis of Solids," presented at the Nuclear Materials and Technology Review, Los Alamos, NM, Spring 1999.

Cris Lewis, Wei Hang, Steve Doorn, Dave Wayne, Vahid Majidi, Fred King, "Characterization of a Pulsed Glow Discharge Laser Ablation System for Direct Chemical Analysis of Solids," presented at the 26th Annual Conference of the Federation of Analytical Chemistry and Spectroscopy Societies Meeting, Vancouver, BC, October 1999.

C. L. Lewis, S. Doorn, V. Majidi, M. A. Moser, W. Hang, and F. L. King, "Characterization of a Pulsed Glow Discharge Laser Ablation System for Direct Chemical Analysis of Solids", presented at the Fifty-Second Southeast Regional American Chemical Society Meeting, Knoxville, TN, October 1999.

C. L. Lewis, G. P. Jackson, S. K. Doorn, V. Majidi, D. Wayne, and F. L. King, "Temporally and Spatially Resolved Diagnostics of Pulsed Glow Discharge Sources", presented at the Fifty-Second Southeast Regional American Chemical Society Meeting, Knoxville, TN, October 1999.

Vahid Maiidi, Matt Moser, Cris Lewis, Wei Hang, and Fred King, "Complete Chemical Speciation by Low Pressure Tunable Plasmas" ", presented at the Winter Conference on Plasma Spectrochemistry, Fort Lauderdale, Florida, January 2000

Cris Lewis, Stephen Doorn, Vahid Majidi and Fred King, "Optical Emission Characterization of a Pulsed Glow Discharge Laser Ablation System", presented at the Winter Conference on Plasma Spectrochemistry, Fort Lauderdale, Florida, January 2000.

C. L. Lewis, G. P. Jackson, S. K. Doorn, V. Majidi, D. Wayne, and F. L. King, "Temporally and Spatially Resolved Diagnostics of Pulsed Glow Discharge Sources", presented at the Winter Conference on Plasma Spectrochemistry, Fort Lauderdale, Florida, January 2000. 
Matt Moser, Cris Lewis, Wei Hang, Fred L. King and Vahid Majidi, "Acquisition of Elemental, Structural, and Molecular Information Using a Microsecond Pulsed Glow Discharge" presented at the Winter Conference on Plasma Spectrochemistry, Fort Lauderdale, Florida, January 2000. 